\title{
Surface photometry of binary galaxies. \\ I. A multicolour study of morphologies due to the interaction ${ }^{\star}$
}

\author{
L. Reduzzi ${ }^{1}$ and R. Rampazzo ${ }^{2}$ \\ 1 Istituto di Fisica Applicata, Università di Milano, Via Brera 28, I-20121 Milano, Italy \\ 2 Osservatorio Astronomico di Brera, Via Brera 28, I-20121, Milano, Italy
}

Received June 22; accepted October 2, 1995

\begin{abstract}
We present the multicolour $(B, V, R)$ surface photometry of 53 double galaxies. All the pairs belong to the catalogue of Reduzzi \& Rampazzo (1995) containing objects selected according to the Karachentsev (1972) criteria. We comment on the morphological, structural and photometric characteristics of pairs and their members. Different classes of interaction induced phenomena, both among early and late-type galaxies, are considered. We found that few early-type galaxies show fine structures. Grand design structure is more frequently detected in binary than in field spirals both for barred and non barred, confirming Elmegreen \& Elmegreen's (1982) study. The colour of the tails is consistent with the stripping hypothesis since it is similar to the progenitor galaxy outskirts. Among our objects we have no evident sign of induced star formation in tails. Rings appear on average bluer than the disc as a whole.
\end{abstract}

Key words: galaxies: photometry — galaxies: elliptical and lenticular — galaxies: spiral — galaxies: interactions — galaxies: evolution

\section{Introduction}

Beginning in the '70s numerical simulations of encounters (Toomre \& Toomre 1972; Barnes \& Hernquist 1992 and reference therein) and the development of photometric models (Larson \& Tinsley 1978; Kennicutt 1990 and references therein) both indicated that galaxy evolution is modified by interactions. This suggested a connection between the morphological disturbance and the different levels of activity (from nuclear activity to star formation, SF hereafter, enhancement) in galaxies. This connection is not yet completely understood and a clear correlation between morphological distortions and sign of activity does not yet exist (Dahari \& De Robertis 1988).

The presence of strong SF processes in galaxies with nuclear activity indicates that nuclear activity, enhanced $\mathrm{SF}$ and gravitational interaction are connected properties. Recently, Moles et al. (1995) studied the morphology of the galaxies showing Seyfert or LINER activity in the Véron-Cetty \& Véron (1991) catalogue concluding that what "seems necessary for the nuclear activity to appear is the ability of a given galaxy to produce a global, well organized response to the nonaxisymmetric component of the potential. This could explain why active galaxies are not so frequent among strongly interacting systems, where

Send offprint requests to: L. Reduzzi

${ }^{\star}$ Based on observations obtained at ESO, La Silla, Chile the galaxy is torn apart." Nuclei and rings seem privileged places of SF, but the dispersion in the colour of the rings is due also to the gas richness of the galaxy. Pogge \& Eskridge (1993) found that HI rich S0s either show $\mathrm{H} \alpha$ emissions with a ring-like structure or are concentrated in the centre. The bar, in fact, tends to remove the gas kinetic energy and angular momentum driving the gas towards the centre. Rampazzo et al. (1995), studying a sample of interacting pairs, showed that LINER phenomena and a shock induced inversion of the ratio $[\mathrm{NII}] / \mathrm{H} \alpha$ can happen in the very centre of the nuclei, typically inside 1 $\mathrm{Kpc}$, in conjunction with the presence of a bar. Marston \& Appleton (1995), studying asymmetric rings, probably due to a head-on encounter, found that "ring galaxies have similar $\mathrm{H} \alpha$ luminosities as starburst galaxies, with star formation being almost exclusively concentrated in the ring".

Since the detailed local mechanisms of star-formation are not known, simulations of interacting galaxy encounters formulate different expectations concerning SF development and SF loci during the collision. Several groups have tried to quantify the SF enhancement due to tidal perturbations adopting different SF laws. Noguchi \& Ishibashi (1986) adopted a star formation linked to cloudcloud collisions. They found that the SF rate could be enhanced by a factor 6 after pericentre, during a close passage. The gas collects in the centre and in a transient ring. The order of magnitude enhancement they 
found was the same as observed by Bushouse (1987) in $\mathrm{H} \alpha$. Olson \& Kwan (1990a, b) assumed that the SF follows the disruption of giant molecular clouds by collisions. Adopting a SF efficiency, they obtain reasonable agreement with the observed luminosity-to-gas ratios in interacting and merger systems. However, they can account for the highest ratios only by considering the gas depletion into star formation. The increase in SF efficiency is then not due to an increase in burst luminosity, but to a gas content decrease. This does not take into account possible gas infall to fuel the star-burst, as suggested by the Combes et al. (1994) result of enhanced molecular content in interacting galaxies. Mihos et al. (1991) in their simulations adopted a Schmidt law for the SF rate. They found that most of the time the SF rate is enhanced in interacting galaxies, since the gas is gathered in spiral arms, and driven towards the centre. In some cases, the gas is so depleted in tidal tails that the $\mathrm{SF}$ rate is decreased with respect to an isolated galaxy. However, they have not included self-gravity, which considerably underestimates radial gas flows.

Thus, we still need observations in order to answer some basic questions:

1) Is it possible to define the necessary and sufficient conditions which connect the activity to the morphological features produced by the interaction? It is clear that a more quantitative morphological classification of galaxies and features is needed.

2) What dynamical conditions may induce/enhance the activity? When is SF enhanced during an interaction episode (just after the perigalactic passage or later)? Do we also observe a dimming in the SF?

3) Where does SF occur (in the circumnuclear region as in some prototypical galaxy with nuclear activity (Heckman et al. 1986), in the ring, in the disc, etc.)? How is this connected with the amount of gas in the interacting members?

4) What are the mechanisms through which the different phenomena are activated (bar and gas driven mechanisms (see Combes et al. 1994), cloud fragmentation, etc.)?

Most of the above questions require a statistical approach on a carefully selected sample. Due to their environmental simplicity, compared to that of groups or clusters, isolated binary galaxies provide a unique opportunity to study galaxies and their sub-components (stellar bulge and disc, gas and dust), in a non-equilibrium configuration. The compilations of isolated double galaxies tend to select nearby, typically interacting, galaxies. The aim of such samples is to isolate the population of binaries for which the potential energy of interaction is by far larger than the interaction energy with any other nearby galaxy. Pairs may then offer several pieces of information about the questions sketched above.
Table 1. Journal of observations

\begin{tabular}{|c|c|c|c|c|}
\hline $\begin{array}{l}\text { RR95 Pair } \\
\text { number }\end{array}$ & $\begin{array}{c}\text { ESO-LV ident. } \\
\text { a/b comp. }\end{array}$ & $\begin{array}{l}\text { CCD field } \\
\qquad\left({ }^{\prime} x^{\prime}\right)\end{array}$ & $\begin{array}{l}\text { Exp. time } \\
\text { (minutes) }\end{array}$ & \\
\hline 24 & E2440120/1 & $3.5 \times 2.3$ & $30,15,10$ & $B, V, R$ \\
\hline 62 & $\mathrm{E} 5450400 / 410$ & $3.5 \times 2.3$ & $40,20,15$ & $B, V, R$ \\
\hline 94 & E1190130/140 & $3.5 \times 2.3$ & $40,20,15$ & $B, V, R$ \\
\hline 98 & $\mathrm{E} 330040 / 50$ & $3.5 \times 2.3$ & $30,20,15$ & $B, V, R$ \\
\hline 99 & $\mathrm{E} 5520490 / 500$ & $3.5 \times 2.3$ & $40,20,15$ & $B, V, R$ \\
\hline $100 \mathrm{a}$ & E3610250 & $3.5 \times 2.3$ & $30,20,15$ & $B, V, R$ \\
\hline $100 \mathrm{~b}$ & E3620010 & $3.5 \times 2.3$ & 20 & $R$ \\
\hline 101 & $\mathrm{E} 4860170 / 190$ & $3.5 \times 2.3$ & $30,15,10$ & $B, V, R$ \\
\hline 105 & $\mathrm{E} 4860290 / 300$ & $3.5 \times 2.3$ & $30,15,10$ & $B, V, R$ \\
\hline $106 \mathrm{a}$ & $\mathrm{E} 4220370$ & $3.5 \times 2.3$ & $30,15,15$ & $B, V, R$ \\
\hline $106 \mathrm{~b}$ & E4220390 & $3.5 \times 2.3$ & $30,15,10$ & $B, V, R$ \\
\hline 120 & E160090/100 & $3.5 \times 2.3$ & $40,20,15$ & $B, V, R$ \\
\hline 129 & E3640350/360 & $3.5 \times 2.3$ & $30,15,10$ & $B, V, R$ \\
\hline 131 & $\mathrm{E} 5560050 / 60$ & $3.5 \times 2.3$ & $30,15,10$ & $B, V, R$ \\
\hline 133 & $\mathrm{E} 5560130 / 140$ & $3.5 \times 2.3$ & $40,20,15$ & $B, V, R$ \\
\hline 136 & E3650280/290 & $3.5 \times 2.3$ & $30,15,10$ & $B, V, R$ \\
\hline $143 \mathrm{a}$ & E870440 & $3.5 \times 2.3$ & 30,7 & $B, R$ \\
\hline $143 \mathrm{~b}$ & $\mathrm{E} 870450$ & $3.5 \times 2.3$ & $30,15,10$ & $B, V, R$ \\
\hline 153 & E1230110/120 & $3.5 \times 2.3$ & $40,20,9$ & $B, V, R$ \\
\hline 155 & $\mathrm{E} 880230 / 240$ & $3.5 \times 2.3$ & $30,15,10$ & $B, V, R$ \\
\hline 161 & E1240180/190 & $3.8 \times 3.8$ & $30,20,15$ & $B, V, R$ \\
\hline 162 & E5640100/110 & $3.5 \times 2.3$ & $40,20,10$ & $B, V, R$ \\
\hline 175 & $\mathrm{E} 2630030 / 31$ & $3.8 \times 3.8$ & $45,25,15$ & $B, V, R$ \\
\hline 177 & E3160320/330 & $3.8 \times 3.8$ & $40,25,15$ & $B, V, R$ \\
\hline 181 & $\mathrm{E} 56705$ & & & $B, V, R$ \\
\hline 182 & E3170180/200 & $3.5 \times 2.3$ & $30,15,10$ & $B, V, R$ \\
\hline 184 & E4360180/190 & $3.5 \times 2.3$ & $30,15,10$ & $B, V, R$ \\
\hline $187 \mathrm{a}$ & E3750640 & $3.5 \times 2.3$ & $30,15,10$ & $B, V, R$ \\
\hline $187 \mathrm{~b}$ & E3750650 & $3.5 \times 2.3$ & $30,10,10$ & $B, V, R$ \\
\hline 197 & E4380 & & & $B, V, R$ \\
\hline 204 & E3790200/210 & $3.5 \times 2.3$ & $30,15,10$ & $B, V, R$ \\
\hline 210 & $\mathrm{E} 4400540 / 560$ & $3.8 \times 3.8$ & $20,25,15$ & $B, V, R$ \\
\hline 211 & $\mathrm{E} 3210060 / 70$ & $3.8 \times 3.8$ & $40,25,15$ & $B, V, R$ \\
\hline $215 \mathrm{a}$ & E5060010 & $3.5 \times 2.3$ & 40,15 & $B, R$ \\
\hline $215 b$ & E5060020 & $3.5 \times 2.3$ & 10 & $R$ \\
\hline 216 & E3220040/60 & $3.8 \times 3.8$ & $40,25,15$ & $B, V, R$ \\
\hline 217 & E3220190/200 & $3.8 \times 3.8$ & $35,20,10$ & $B, V, R$ \\
\hline 222 & $\mathrm{E} 5070270 / 280$ & $3.8 \times 3.8$ & $30,10,15$ & $B, V, R$ \\
\hline 225 & E5070450/460 & $3.8 \times 3.8$ & $25,15,10$ & $B, V, R$ \\
\hline 244 & E4440270/280 & $3.8 \times 3.8$ & $25,15,10$ & $B, V, R$ \\
\hline 248 & $\mathrm{E} 4440440 / 450$ & $3.8 \times 3.8$ & 45,15 & $B, R$ \\
\hline 250 & E3830140/150 & $3.8 \times 3.8$ & $20,15,10$ & $B, V, R$ \\
\hline 267 & E5100580/590 & $3.8 \times 3.8$ & $30,20,15$ & $B, V, R$ \\
\hline 273 & E2210340/341 & $3.8 \times 3.8$ & $30,20,15$ & $B, V, R$ \\
\hline 280 & E5120180/190 & $3.8 \times 3.8$ & 35,10 & $B, R$ \\
\hline 284 & $\mathrm{E} 5140230 / 240$ & $3.8 \times 3.8$ & $40,30,20$ & $B, V, R$ \\
\hline 285 & E1370440/441 & $3.8 \times 3.8$ & $30,20,15$ & $B, V, R$ \\
\hline
\end{tabular}


Table 1. continued

\begin{tabular}{lccll}
\hline $\begin{array}{l}\text { RR95 Pair } \\
\text { number }\end{array}$ & $\begin{array}{c}\text { ESO-LV ident. } \\
\text { a/b comp. }\end{array}$ & $\begin{array}{c}\text { CCD field } \\
\left({ }^{\prime} \times{ }^{\prime}\right)\end{array}$ & $\begin{array}{c}\text { Exp. time } \\
(\text { minutes })\end{array}$ & \\
\hline 287 & E1380290/300 & $3.8 \times 3.8$ & $40,20,30$ & $B, V, R$ \\
290 & E100010/20 & $3.8 \times 3.8$ & $20,15,10$ & $B, V, R$ \\
$292 \mathrm{a}$ & $\mathrm{E} 1400430$ & $3.8 \times 3.8$ & 45,15 & $B, R$ \\
$292 \mathrm{~b}$ & $\mathrm{E} 1400440$ & $3.8 \times 3.8$ & 30,10 & $B, R$ \\
293 & $\mathrm{E} 1830170 / 180$ & $3.5 \times 2.3$ & 40,15 & $B, R$ \\
296 & $\mathrm{E} 1840100 / 110$ & $3.8 \times 3.8$ & $40,20,15$ & $B, V, R$ \\
297 & $\mathrm{E} 1040360 / 370$ & $3.8 \times 3.8$ & $25,15,10$ & $B, V, R$ \\
298 & $\mathrm{E} 1040451 / 50$ & $3.8 \times 3.8$ & $25,15,15$ & $B, V, R$ \\
307 & $\mathrm{E} 2830190 / 200$ & $3.8 \times 3.8$ & $40,15,15$ & $B, V, R$ \\
317 & $\mathrm{E} 4000290 / 300$ & $3.8 \times 3.8$ & $25,20,15$ & $B, V, R$ \\
322 & $\mathrm{E} 2340590 / 600$ & $3.5 \times 2.3$ & 50,15 & $B, R$ \\
353 & $\mathrm{E} 2880010 / 20$ & $3.8 \times 3.8$ & $25,15,15$ & $B, V, R$ \\
\hline
\end{tabular}

In this paper we present the multicolour photometric study of a sample of 53 pairs selected from Reduzzi \& Rampazzo (1995: RR95 hereafter). Our attention is centred on the detailed galaxy morphology and on the colour of those features that simulations credit to the interaction. The sample contains a wide range of pairs, from gas rich (spiral/spiral) objects to those (early-type/earlytype) containing members where SF can be at threshold levels. The sample is among those more homogeneously selected for the twodimensional study of the colours of features in interacting objects. Previous samples (see e.g. Schombert et al. 1990; Schmidt \& Hintzen 1991) contain a mixture of pairs and multiple objects.

The RR95 catalogue has been extracted from The Surface Photometry Catalogue of the ESO-Uppsala Galaxies (Lauberts \& Valentijn 1989: ESO-LV). The photometric parameters in the ESO-LV catalogue are derived from analysis of plate material. Global parameters are given for more than 15000 galaxies, but the material in the $B$ and $R$ colours, suitable for a morphological classification, cannot give information about detailed structure of the objects, for which deep and high resolution material is needed. Colours give information neither about local variations possibly due to interaction penomena nor on their shape (rings etc.) since only apertures at standard distances are given. Isophotal shape is not given in ESO-LV. Our detailed photometric data then complement ESO-LV information.

In Sect. 2 CCD observations and the reduction technique are presented. In Sect. 3 the general sample properties and the results on single objects are discussed. In Sect. 4 objects are grouped and discussed according to the different topics previously sketched. In particular, the systematics of morphological distortion induced by the interaction and connected signs of the SF activity deduced from colours, are discussed in the light of current models. In a forthcoming paper (Paper II) a detailed profile decomposition analysis and the determination of photometric parameters will be performed with different aims among which the location of early-type members of pairs in the fundamental plane.

\section{Data acquisition and analysis}

A journal of the photometric observations is given in Table 1. The observations were done in different runs between 1992 and 1994. All the objects were imaged using the $0.92 \mathrm{~m}$ ESO-Dutch telescope placed at La Silla, Chile, and with similar instrumental set up. In this sense, our data can be considered as obtained under quite homogeneous observational conditions. The original frames were in the Bessel $b, v$ and $r$ bands.

The image cleaning, dark and bias subtraction, flatfielding, cosmic ray removal and the final calibration and image manipulation were performed using the IRAF package. In Table 2 for each separate frame are given the values of seeing and the sky surface brightness measurements. The latter have been estimated avoiding lowsurface-brightness features, halos of bright stars or any other objects that might degrade the estimate. The table reports also the errors in the sky determination. The zero points are based on measurements of photometric standards. When the night was not fully photometric (see in Table 2 the quality parameter) we used the aperture photometry available in ESO-LV. The standard stars used for calibration purposes were obtained in the fields of Rubin 149, PG09-18, PG13-23, PG16-33, Markarian A, T Phe, SA 98. These fields contain few calibration stars each and crowding was not a problem as in the typical fields surrounding globular cluster areas.

We used the standard photometric transformation equations tailored to the ESO (La Silla) systems and extinction coefficients:

$$
\begin{gathered}
(B-V)=b_{1}+b_{2} *\left(b_{\mathrm{s}}-v_{\mathrm{s}}\right), \\
V=v_{1}+v_{\mathrm{s}}+v_{2} *\left(v_{\mathrm{s}}-r_{\mathrm{s}}\right), \\
(V-R)=r_{1}+r_{2} *\left(v_{\mathrm{s}}-r_{\mathrm{s}}\right),
\end{gathered}
$$

where $b_{\mathrm{s}}, v_{\mathrm{s}}$ and $r_{\mathrm{s}}$ are the instrumental magnitudes in the Bessel bands and $r_{1}, r_{2}, b_{1}, b_{2}, v_{1}, v_{2}$ are the transformation coefficients.

We used a total of 25 standard stars. Their colour range is $-0.296 \leq(B-V) \leq 1.553$ and $-0.149 \leq(V-R)$ $\leq 0.849$. This large range is crucial for any project dealing with star-forming galaxies.

Determination of the luminosity profiles of early-type members were accomplished through interpolation of the isophotes with an ellipse. A different methodology has to be used for spiral galaxies where the presence of arms perturbs any ellipse interpolation (except for the bulge dominated region). We tested the equivalence of two reduction 
strategies that have been discussed in the literature (e.g. Boroson 1981). The first technique derives the luminosity profile as the average of a sample of radial traces at different position angles adequately corrected for inclination. The second one uses an azimuthal profile obtained by fitting ellipses with a fixed position angle and ellipticity previously determined on the external isophotes. While in the external regions these procedures tend to give similar results, both techniques do not produce an adequate profile of the bulge. For this reason we decided that our final profile would be the composite of a completely free azimuthal profile (free selection of the position angle and ellipticity of the isophote) to represent the bulge component and a fixed profile (where the position angle and the ellipticity are defined by the outer isophotes) to describe the galaxy outskirts.

The final results consist of isophotal maps for each pair (Fig. 2) and luminosity plus colour profiles for each member (Fig. 3) . For the early-type members the geometrical profiles (ellipticity, twist, $a 4 / a$ ) are given as well. The deviation from the elliptical shape, described by the $a 4 / a$ parameter, is discussed in the text for each early-type object. The geometrical profiles do not change as a function of the colour. Foreground stars have been removed before any fitting procedure.

Figure 4 presents $2 \mathrm{D}(B-V)$ or $(B-R)$ colour maps for the pairs showing the most evident signs of interaction. In order to control the noise in the low signal regions a Gaussian smoothing $\approx 2^{\prime \prime} \times 2^{\prime \prime}$ has been applied to the colour frame. A continuous scale, rather than colour slices, has been selected in order to represent colour variations in the galaxies and in their features. Colour profiles given in Fig. 3 report the formal errors in the measured colour. Due to the smoothing procedure adopted, in the 2D colour maps the latter can be considered as an upper estimate of the error in the different regions of the galaxy under examination. The foreground stars in the field have not been subtracted from the frame, only spikes, where present, have been masked. The smoothing can then create fictitious patchy regions but they can be easily identified comparing the $2 \mathrm{D}$ colour maps with the original frames in Fig. 2.

Redshifts are available for $\approx 60 \%$ of the objects and since $z \leq 0.025$, no K-correction has been applied to our magnitudes and colours.

We also describe in the text a search for faint structures such as ripples, shells, $X$-structure and dust-lanes (see Schweizer 1992 and references therein) in the early type members of the pairs. The method adopted for enhancing fine structures is discussed in Reduzzi et al. (1994).

\section{Discussion}

\subsection{The sample}

Table 3 reports the salient properties of the observed pairs and their members. In Figs. 1.1 and 1.2 are shown some of the properties of the sample. The ESO-LV catalogue reports the parameter $N_{\text {tot }}$ which corresponds to the total number of galaxies in a square degree around a given galaxy. This is a measure of the surface density, although the authors state that it correlates also with the volume density. Since according to the authors a galaxy is in a rich cluster when $N_{\text {tot }}>9-10$ most of our pairs, see Fig. 1.1, populate very low density environments. Following the classification given in the ESO-LV the morphological type of the members of galaxy pairs cover nearly all the Hubble sequence. There is no indication that the observed pair members have similar morphological types. Actually, as shown in Fig. 1.1, also the regions in which mixed morphological types are present, are rather populated. We know the velocity difference for $\approx 60 \%$ of the sample. Two pairs, namely 292 and 143, have to be considered optical. Because the fraction of optical pairs $(\Delta V>$ $1000 \mathrm{~km} \mathrm{~s}^{-1}$ ) in the RR95 catalogue is $11 \%$, we expect few other cases among the sample here discussed. The separation between the members in the observed sample is very small, $\approx 1$ galaxy diameter $\left(\left\langle a_{25, \mathrm{a}} / a_{25, \mathrm{~b}}\right\rangle=1.2 \pm 0.7\right)$. We are then mainly dealing with tight pairs, although in projection.

\subsection{Comments on individual objects}

In this section we describe the observed features of pairs and their members. This will be the basis for the general morphological distinction and evolution considerations developed in the next Section.

For the classification we follow ESO-LV complemented by RC3 (de Vaucouleurs et al. 1991). Usually there is agreement between the two classification systems; when we notice a difference we report it in the text. In the case of early-type galaxies (from Es to late S0s) we try to parameterize the shape of the luminosity, ellipticity, twist and $a 4 / a$ profiles for a more quantitative classification (see Capaccioli et al. 1990). It can be easily foreseen that some differences will emerge when comparing classification obtained from plates and from high resolution CCD images.

A particular attention will be dedicated to the presence of fine structures in the early-type component. A 'classification' of fine structures has been given by Schweizer (1992). Among the more important for the origin of the early-type galaxies hosting them are ripples/shells since their origin is generally attributed to merging or acquisition phenomena. The origin of other features like isophotal shape (boxiness or disciness: see Bender et al. 1989) are more uncertain (see Stiavelli et al. 1991; Governato et al. 1993). Goudfrooij (1994) suggests that the dust in early-type galaxies, as opposed to spirals, has probably an 
Table 2. Sky values and seeing conditions

\begin{tabular}{|c|c|c|c|c|c|c|c|c|}
\hline \multirow{2}{*}{$\begin{array}{l}\text { RR95 Pair } \\
\text { number }\end{array}$} & \multirow{2}{*}{$\begin{array}{l}\text { ESO-LV ident. } \\
\text { a/b comp. }\end{array}$} & \multicolumn{3}{|c|}{ Seeing $\left({ }^{\prime \prime}\right)$} & \multicolumn{3}{|c|}{ Sky $\left(\operatorname{mag} \operatorname{arcsec}^{-2}\right)$} & \multirow[t]{2}{*}{ Notes } \\
\hline & & $\sigma_{B}$ & $\sigma_{V}$ & $\sigma_{R}$ & $B$ & $V$ & $R$ & \\
\hline 24 & $\mathrm{E} 2440120 / 1$ & 2.1 & 2.0 & 2.0 & $21.94 \pm .13$ & $21.45 \pm .08$ & $20.03 \pm .03$ & $1, \mathrm{NP}$ \\
\hline 62 & E5450400/410 & 2.3 & 2.1 & 2.0 & $21.63 \pm .12$ & $20.91 \pm .07$ & $20.39 \pm .05$ & 1 \\
\hline \multirow[t]{2}{*}{94} & \multirow[t]{2}{*}{ E1190130/140 } & 2.2 & 1.7 & 1.9 & $21.91 \pm .10$ & $21.33 \pm .07$ & $20.48 \pm .02$ & 1,a comp. \\
\hline & & 1.3 & 1.3 & 1.3 & $21.95 \pm .10$ & $21.60 \pm .08$ & $20.85 \pm .03$ & 1,b comp. \\
\hline 98 & $\mathrm{E} 330040 / 50$ & 2.1 & 1.6 & 1.6 & $20.92 \pm .08$ & $20.84 \pm .06$ & $20.11 \pm .03$ & 1 \\
\hline 99 & E5520490/500 & 2.1 & 1.8 & 1.7 & $21.65 \pm .09$ & $21.12 \pm .07$ & $20.57 \pm .03$ & 1 \\
\hline 100 & E3610250/3620010 & 2.2 & 1.9 & $\begin{array}{l}1.4 \\
1.5\end{array}$ & $21.68 \pm .13$ & $21.19 \pm .06$ & $\begin{array}{l}20.67 \pm .03 \\
20.72 \pm .04\end{array}$ & $\begin{array}{l}2, \text { a comp. } \\
2, \text { b comp. }\end{array}$ \\
\hline 101 & E4860170/190 & 1.5 & 1.7 & 1.8 & $21.56 \pm .12$ & $21.12 \pm .08$ & $20.73 \pm .04$ & 1 \\
\hline 105 & E4860290/300 & 1.8 & 1.8 & 1.8 & $21.59 \pm .12$ & $21.04 \pm .08$ & $20.43 \pm .04$ & 1 \\
\hline \multirow[t]{2}{*}{106} & \multirow{2}{*}{$\mathrm{E} 4220370 / 390$} & 2.0 & 1.7 & 1.9 & $21.54 \pm .11$ & $21.18 \pm .09$ & $20.62 \pm .04$ & 2,a comp. \\
\hline & & 1.7 & 1.5 & 1.5 & $21.20 \pm .09$ & $20.83 \pm .07$ & $20.71 \pm .03$ & 2,b comp. \\
\hline \multirow[t]{2}{*}{120} & \multirow[t]{2}{*}{ E160090/100 } & 2.0 & 1.9 & 2.1 & $21.23 \pm .08$ & $20.60 \pm .06$ & $20.16 \pm .03$ & 2, a comp. \\
\hline & & 2.1 & 1.7 & 1.7 & $20.63 \pm .05$ & $20.62 \pm .02$ & $19.84 \pm .02$ & 2,b comp. \\
\hline 129 & E3640350/360 & 1.5 & 1.3 & 1.3 & $21.45 \pm .12$ & $21.03 \pm .11$ & $20.45 \pm .06$ & 1 \\
\hline 131 & E5560050/60 & 2.3 & 2.2 & 2.0 & $21.31 \pm .10$ & $20.84 \pm .07$ & $20.35 \pm .03$ & 1 \\
\hline 133 & E5560130/140 & 1.7 & 1.6 & 1.6 & $21.37 \pm .09$ & $20.72 \pm .05$ & $20.42 \pm .03$ & 1 \\
\hline 136 & E3650280/290 & 1.6 & 1.7 & 1.9 & $21.61 \pm .12$ & $21.01 \pm .07$ & $20.54 \pm .04$ & 1 \\
\hline \multirow[t]{2}{*}{143} & \multirow[t]{2}{*}{$\mathrm{E} 870440 / 450$} & 1.3 & & 1.3 & $21.38 \pm .10$ & & $20.49 \pm .05$ & 1, a comp. optical \\
\hline & & 1.3 & 1.3 & 1.2 & $21.50 \pm .10$ & $21.18 \pm .08$ & $20.51 \pm .04$ & 1,b comp. \\
\hline 153 & E1230110/120 & 1.6 & 1.5 & 1.6 & $21.56 \pm .09$ & $20.83 \pm .05$ & $20.26 \pm .03$ & 1 \\
\hline \multirow[t]{2}{*}{155} & \multirow[t]{2}{*}{$\mathrm{E} 880230 / 240$} & 1.6 & 1.6 & 1.5 & $21.45 \pm .12$ & $20.97 \pm .09$ & $20.51 \pm .04$ & 3,a comp. \\
\hline & & 1.5 & 1.5 & 1.4 & $21.43 \pm .11$ & $20.96 \pm .07$ & $20.30 \pm .03$ & 3,b comp. \\
\hline 161 & E1240180/190 & 1.6 & 1.5 & 1.5 & $22.55 \pm .05$ & $21.40 \pm .03$ & $20.53 \pm .02$ & 1 \\
\hline 162 & E5640100/110 & 1.6 & 1.5 & 1.5 & $21.41 \pm .09$ & $20.47 \pm .05$ & $20.24 \pm .03$ & 1 \\
\hline 175 & E2630030/31 & 1.6 & 1.6 & 1.4 & $22.53 \pm .04$ & $21.48 \pm .03$ & $20.67 \pm .02$ & $2, \mathrm{NP}$ \\
\hline 177 & E3160320/330 & 1.6 & 1.5 & 1.4 & $22.84 \pm .05$ & $21.69 \pm .03$ & $20.80 \pm .03$ & 1 \\
\hline 181 & E5670520/530 & 1.4 & 1.3 & 1.4 & $22.70 \pm .04$ & $21.64 \pm .02$ & $20.72 \pm .02$ & 1 \\
\hline 182 & E3170180/200 & 1.6 & 1.5 & 1.5 & $21.64 \pm .11$ & $21.13 \pm .06$ & $20.35 \pm .03$ & 1,b comp. \\
\hline \multirow[t]{2}{*}{184} & \multirow[t]{2}{*}{ E4360180/190 } & 1.3 & 1.2 & 1.2 & $21.57 \pm .11$ & $21.15 \pm .08$ & $20.50 \pm .04$ & 1,a comp. \\
\hline & & 1.4 & 1.2 & 1.1 & $21.63 \pm .11$ & $21.19 \pm .09$ & $20.56 \pm .04$ & 1,b comp. \\
\hline \multirow[t]{2}{*}{187} & \multirow[t]{2}{*}{ E3750640/650 } & 1.4 & 1.3 & 1.2 & $21.58 \pm .11$ & $21.11 \pm .07$ & $20.42 \pm .04$ & 1,a comp. \\
\hline & & 1.5 & 1.3 & 1.4 & $21.61 \pm .12$ & $21.17 \pm .10$ & $20.50 \pm .04$ & 1,b comp. \\
\hline
\end{tabular}

external origin since the mass of the cool dust is independent of the optical luminosity of the galaxy. $X$-structure is among the most rare features and its origin is also attributed to the merging of two galaxies (see Binney \& Petrou 1985; Hernquist \& Quinn 1989). We are aware that Forbes \& Thomson (1992) found that the detection of this latter feature is connected in a tangled way to boxy isophotes. For this reason, attention has been paid in order to disentangle real from spurious effects.

Median integrated total $(B-V)$ colours of galaxies according to their morphological class are given in Roberts \& Haynes (1994: RH94 hereafter). In their Table 1 the median $(B-V)$ has a monotonic decrease from E-S0 (0.90), to Sm-Im (0.42) through S0a-Sa (0.78), Sab-Sb (0.64), SbcSc (0.55) and Scd-Sd (0.48). RH94 report that the "range of colour among Sa galaxies overlaps that of Sc: some Scs are as red as some Sas and some Sas as blue as Scs". The samples used by RH94 are rather inhomogeneous in terms of environment but the interacting objects were excluded from their analysis. So they interpreted the spread in $(B-V)$ colour as presumably due to current SF rates in individual objects. Balcells \& Peletier (1994) studied colours and colour profiles of the bulges for a set of earlytype spirals. The average central colour $(B-R)$ for 18 objects is $1.54 \pm 0.12$. $(B-R)$ colour profiles show different trends. Some objects have central values that reach $(B-R) \approx 2$ even if the measure is obtained on the less contaminated side (e.g. along the minor axis). Also in the Balcells \& Peletier work interacting galaxies have been avoided. Thus we may consider the above ones as comparison and reference samples. 
Table 2. continued

\begin{tabular}{|c|c|c|c|c|c|c|c|c|}
\hline \multirow{2}{*}{$\begin{array}{l}\text { RR95 Pair } \\
\text { number }\end{array}$} & \multirow{2}{*}{$\begin{array}{l}\text { ESO-LV ident. } \\
\text { a/b comp. }\end{array}$} & \multicolumn{3}{|c|}{ Seeing $\left({ }^{\prime \prime}\right)$} & \multicolumn{3}{|c|}{$\operatorname{Sky}\left(\operatorname{mag} \operatorname{arcsec}^{-2}\right)$} & \multirow[t]{2}{*}{ Notes } \\
\hline & & $\sigma_{B}$ & $\sigma_{V}$ & $\sigma_{R}$ & $B$ & $V$ & $R$ & \\
\hline 197 & $\mathrm{E} 4380200 / 210$ & 1.9 & 1.5 & 1.5 & $21.66 \pm .09$ & $20.93 \pm .07$ & $20.51 \pm .03$ & 1 \\
\hline 204 & E3790200/210 & 1.4 & 1.6 & 1.7 & $21.54 \pm .11$ & $21.00 \pm .07$ & $20.35 \pm .04$ & 2 \\
\hline 210 & $\mathrm{E} 4400540 / 560$ & 1.8 & 1.6 & 1.5 & $20.49 \pm .02$ & $19.95 \pm .01$ & $19.73 \pm .01$ & 3 \\
\hline 211 & $\mathrm{E} 3210060 / 70$ & 1.7 & 1.7 & 1.6 & $22.57 \pm .05$ & \multirow[t]{2}{*}{$21.20 \pm .02$} & $20.51 \pm .02$ & 2 \\
\hline 215 & E5060010/20 & 2.1 & & $\begin{array}{l}1.9 \\
1.6\end{array}$ & $21.94 \pm .13$ & & $\begin{array}{l}20.14 \pm .03 \\
20.47 \pm .05\end{array}$ & $\begin{array}{l}\text { 1, a comp. } \\
\text { 1,b comp. }\end{array}$ \\
\hline 216 & E3220040/60 & 1.6 & 1.5 & 1.4 & $22.65 \pm .05$ & $21.72 \pm .03$ & $20.84 \pm .02$ & 1 \\
\hline 217 & E3220190/200 & 1.6 & 1.5 & 1.5 & $22.59 \pm .05$ & $21.62 \pm .02$ & $20.92 \pm .03$ & 2 \\
\hline 222 & E5070270/280 & 1.6 & 1.5 & 1.4 & $22.80 \pm .05$ & $21.62 \pm .04$ & $20.82 \pm .02$ & 2 \\
\hline 225 & E5070450/460 & 1.5 & 1.4 & 1.3 & $22.61 \pm .06$ & $21.37 \pm .03$ & $20.63 \pm .02$ & 2 \\
\hline 244 & $\mathrm{E} 4440270 / 280$ & 1.6 & 1.6 & 1.5 & $22.27 \pm .05$ & $21.45 \pm .03$ & $20.93 \pm .03$ & 1 \\
\hline 248 & $\mathrm{E} 4440440 / 450$ & 2.4 & & 1.8 & $22.17 \pm .04$ & & $20.95 \pm .03$ & $1, \mathrm{NP}$ \\
\hline 250 & E3830140/150 & 1.6 & 1.5 & 1.5 & $22.76 \pm .07$ & $21.79 \pm .03$ & $20.97 \pm .02$ & 1 \\
\hline 267 & E5100580/590 & 1.6 & 1.7 & 1.6 & $22.58 \pm .04$ & $21.58 \pm .03$ & $20.84 \pm .02$ & 1 \\
\hline 273 & E2210340/341 & 1.7 & 1.6 & 1.6 & $22.04 \pm .04$ & $21.10 \pm .02$ & $20.48 \pm .02$ & 2 \\
\hline 280 & E5120180/190 & 1.6 & & 1.4 & $22.07 \pm .04$ & & $20.63 \pm .03$ & $1, \mathrm{NP}$ \\
\hline 284 & E5140230/240 & 1.6 & 1.5 & 1.5 & $22.48 \pm .04$ & $21.39 \pm .02$ & $20.61 \pm .02$ & 2 \\
\hline 285 & E1370440/441 & 1.7 & 1.6 & 1.6 & $22.41 \pm .05$ & $21.41 \pm .02$ & $20.61 \pm .02$ & 2 \\
\hline 287 & E1380290/300 & 1.6 & 1.4 & 1.4 & $22.48 \pm .04$ & $21.39 \pm .02$ & $20.57 \pm .02$ & 2 \\
\hline 290 & $\mathrm{E} 100010 / 20$ & 1.5 & 1.4 & 1.4 & $22.33 \pm .05$ & $21.19 \pm .03$ & $20.45 \pm .02$ & $2, \mathrm{NP}$ \\
\hline \multirow[t]{2}{*}{292} & \multirow[t]{2}{*}{ E1400430/440 } & 2.1 & & 2.3 & $22.05 \pm .03$ & & $20.48 \pm .02$ & 2 ,a comp.optical \\
\hline & & 2.5 & & 2.3 & $21.81 \pm .05$ & & $20.41 \pm .03$ & 2,b comp. \\
\hline 293 & E1830170/180 & 2.4 & & 1.9 & $21.44 \pm .11$ & & $20.02 \pm .03$ & 1 \\
\hline 296 & E1840100/110 & 1.5 & 1.4 & 1.3 & $22.55 \pm .04$ & $21.35 \pm .02$ & $20.59 \pm .02$ & 1 \\
\hline 297 & $\mathrm{E} 1040360 / 370$ & 1.6 & 1.6 & 1.6 & $22.41 \pm .06$ & $21.37 \pm .04$ & $20.59 \pm .03$ & 1 \\
\hline 298 & $\mathrm{E} 1040451 / 50$ & 1.9 & 1.6 & 1.7 & $22.48 \pm .06$ & $21.24 \pm .03$ & $20.18 \pm .02$ & 1 \\
\hline 307 & E2830190/200 & 1.6 & & 1.5 & $22.14 \pm .04$ & & $20.52 \pm .01$ & 1 \\
\hline 317 & E4000290/300 & 1.6 & 1.4 & 1.3 & $22.53 \pm .06$ & $21.49 \pm .02$ & $20.67 \pm .02$ & 1 \\
\hline \multirow[t]{2}{*}{322} & \multirow[t]{2}{*}{$\mathrm{E} 2340590 / 600$} & 2.1 & & 2.2 & $21.59 \pm .13$ & & $20.17 \pm .03$ & 1,a comp. \\
\hline & & 2.2 & & 1.7 & $21.64 \pm .14$ & & $19.88 \pm .02$ & 1,b comp. \\
\hline 353 & $\mathrm{E} 2880010 / 20$ & 1.6 & 1.5 & 1.3 & $22.23 \pm .05$ & $21.28 \pm .03$ & $20.16 \pm .02$ & 1 \\
\hline
\end{tabular}

Notes: an estimate of the photometric quality of the night is given in the last column with the following legenda: $1=$ good; $2=$ intermediate; $3=$ low. NP indicates that the luminosity profile has not been extracted (see individual notes for an explanation).

Pair 24 (AM0115-444). Due to the small separation of the galaxies we did not compute the luminosity profiles. In fact, each member is strongly contaminated by the light of the companion. The complexity in the isophotal structure prevented us from modelling each member and then to try an iterative subtraction. One of the object is classified as $\mathrm{Sb}$ while the second is a lenticular. The 2D $(B-V)$ map shows that the colour of the two objects is quite similar and consistent with the late type morphological classification. The distorted structure of the arms suggests that they are probably created/modified by the interaction, but their $(B-V)$ colour is indistinguishable from that of a normal spiral.

Pair 62. 62a is classified as a lenticular but the luminosity profile can be well represented by an $r^{1 / 4}$ law. Outside the region contaminated by the seeing, between $5^{\prime \prime}$ and $10^{\prime \prime}$, a small deviation from the de Vaucouleurs law is visible in the luminosity profile in correspondence to a variation of the ellipticity trend. In the same region $62 \mathrm{a}$ is up to $1 \%$ boxy, while outside the $a 4 / a$ profile is quite irregular. The twist is less than $10^{\circ} .62 \mathrm{~b}$ is a spiral ( $\mathrm{Sb}$ in the ESO-LV classification) seen nearly edge-on and the exponential disc is clearly visible. The pair is then a typical $\mathrm{E}+\mathrm{S}$ and the colour profiles are typical of the two classes.

Pair 94 (AM0449-612/AM0450-612). This is a hierarchical pair with no obvious sign of interaction. The ESO-LV classifies the members as late spirals (Sbc/SIrr). 94a shows a prominent bar, visible also in the luminosity profile, from which arms start. The spiral structure is grand design. In 94b the disc is predominant as seen in the profile. Colour profiles are typical of their morphological classes according to RH94. 
Pair 98 (AM0459-753). Sbc+Sa pair following the ESO-LV classification. Arp \& Madore (1987: AM87 hereafter) suggest that the pair is probably part of a quartet. 98a is a spiral with grand design structure. Colours are typical of spirals in all galaxy regions. The $(B-V)$ colour map shows that arms are bluer although foreground stars contaminate the field. 98b shows an exponential disc and, outside the seeing disc, a twist less than $10^{\circ}$. No arms are visible in our frame since the galaxy is seen at high inclination. The colour is quite red also in the outskirts; we suspect strong absorption features caused by dust whose presence can be deduced by the asymmetries of the isophotes (more compressed in the South-Eastern side). This fact influenced also the measure of the $a 4 / a$ profile which appears strongly discy (up to $8 \%$ ).
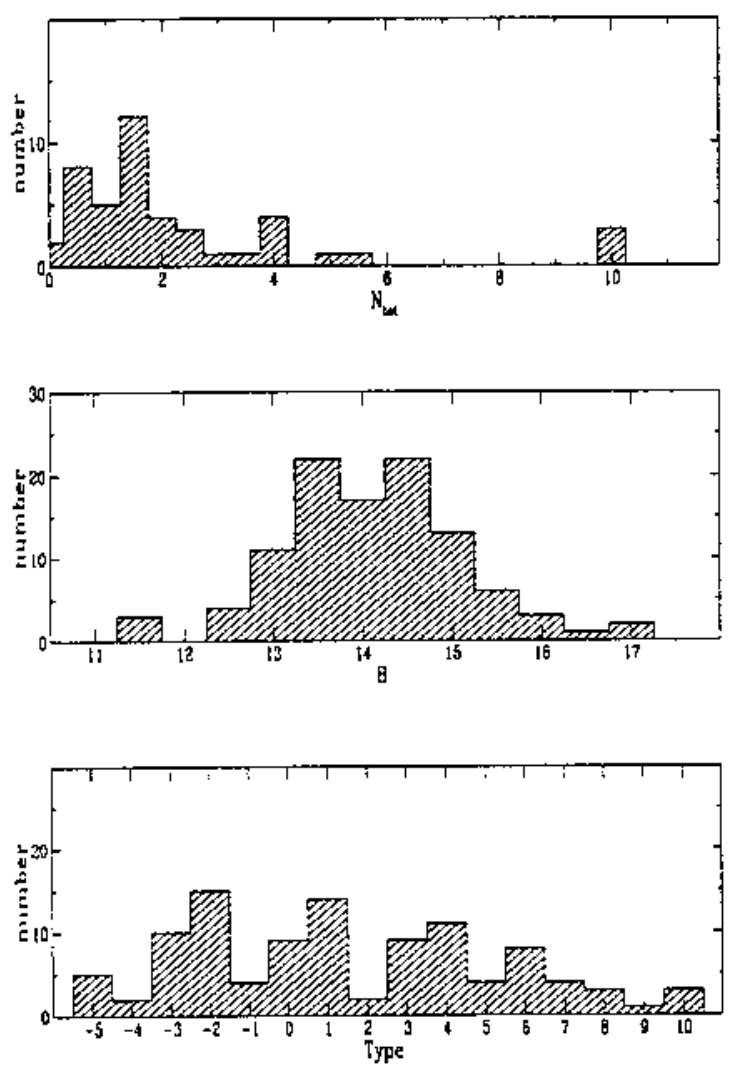

Fig. 1. 1. Properties of the galaxies members of the observed sample of pairs. (top panel) Surface density around members of pairs (see text). (mid panel) Distribution of the total apparent $B$ magnitude. (bottom panel) Distribution of the morphological types. All the parameters were obtained from the ESO-LV

Pair 99 (NGC 1738/39). Interacting Sb+Sbc pair. In both profiles an exponential disc is visible. 99a is very disturbed with multiple arms. 99b shows a bar in the centre; its colour profile is flat and blue also in the centre unlike the companion which has a nearly similar morphological type.
Pair 100 (AM0459-340). In the RR95 catalogue this object is classified as a triplet (see also AM87) since a small galaxy North of 100a is also interacting with it. This latter is not listed in the ESO-LV catalogue because its diameter is less than $1^{\prime}$. A bridge of matter links it to 100a. RC3 classifies 100a as a uncertain spiral. The luminosity profile of 100a has structures (a bar?) superimposed. The colour profile is blue along all the galaxy with the exclusion of the very centre (the bar?) as is clearly visible in the 2D colour map. This latter shows that the stretched arm/tail is blue $((B-V) \approx 0.5)$ with clumpy structure. $100 \mathrm{~b}$ is less luminous than the companion. The bulge is well developed. The luminosity profile can be basically fitted with an $r^{1 / 4}$ law although there is a residual structure and, together with the arms, it justifies the ESO-LV classification as S0a. The arms are quite open and diffused; a significant twist is visible from the isophotal contour.

Pair 101 (AM0501-225). Following the ESO-LV classification, 101 is composed of an E/S0+E. Luminosity profiles confirm such classification. The pair does not show evidence of interaction. In both objects the isophotes are strongly discy (larger than $10 \%$ in 101a and up to $5 \%$ in 101b) as visible from the luminosity, the $a 4 / a$ and the ellipticity profiles. The isophotes are not significantly twisted. The colour profile of 101a tends to be bluer in the outer parts.

Pair 105 (AM0505-230). In the ESO-LV the pair members are classified E/S0 and Sd. The colour profile of $105 \mathrm{a}$ is typical of an early-type galaxy and can be fitted with an $r^{1 / 4}$ law. No fine structures are visible since a model made with the parameters shown in Fig. 3 perfectly subtracts the galaxy. 105b is a spiral where the disc predominates. The disc shows asymmetries in the light distribution. The case is similar to that in 204b.

Pair 106 (NGC 1811/12) (AM0506-292). Sa+Sa pair in the ESO-LV. 106a has a dust-lane along the galactic plane. Its colour profile is stable and redder than the companion 106b which has the same morphological classification but no visible sign of dust. In fact, the colour profile of 106b becomes very blue in the outskirts for an average Sa indicating active star formation. 106b has a bar in the centre and a ring.

Pair 120 (NGC -/2144) (AM0545-820). Isolated, hierarchical and very separated (8.5) $\mathrm{Sd}+\mathrm{Sa}$ pair. The fainter member, $120 \mathrm{a}$, is very disturbed. The arms are like tails, but an underlying disc is visible. The luminosity profile of $120 \mathrm{~b}$ can be fitted with an $r^{1 / 4}$ law but a luminosity structure is visible in the residuals. The colour profile is quite noisy and red suggesting that diffuse dust can be present. The isophotes of $120 \mathrm{~b}$, the dominant member, are asymmetric in the East-West direction (faint arms?). The colour profile is more similar to that of an early-type galaxy and the luminosity profile can easily be fitted by an $r^{1 / 4}$ law, although significant residuals are present. 

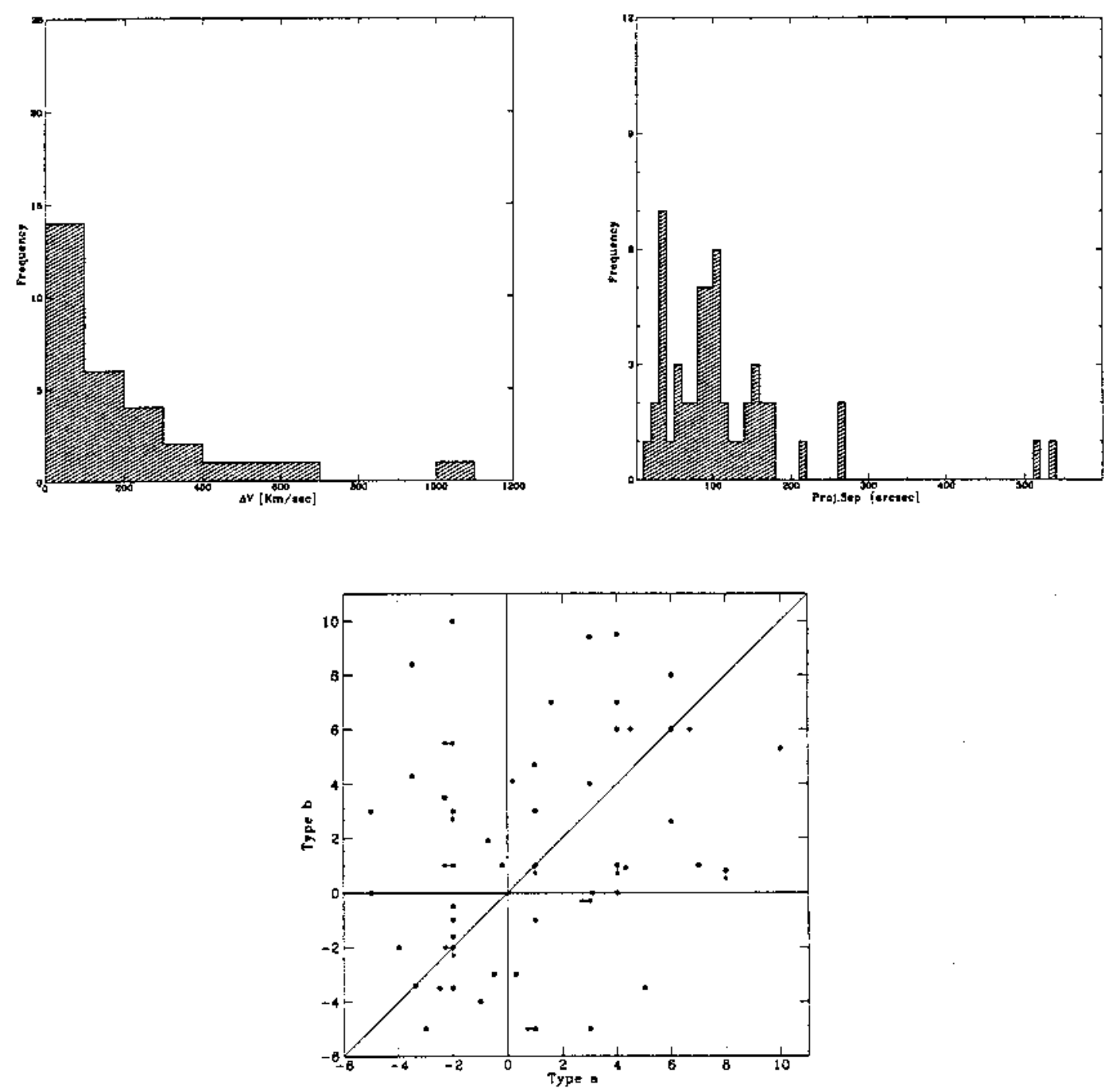

Fig. 1. 2. Properties of the observed sample of pairs. (left panel) Distribution of $\Delta V$. This latter is known for $\approx 60 \%$ of the observed sample. One pair has $\Delta V>3000 \mathrm{~km} \mathrm{~s}^{-1}$ (not in the plot), one has $\Delta V>1000 \mathrm{~km} \mathrm{~s}^{-1}$, while the remaining have velocity differences compatible with those adopted in N-body simulations of encounters to reproduce features really observed in interacting pairs. (right panel). Distribution of the projected separations. The bulk of the objects have separation comparable with their optical radius (see Table 3). (bottom panel) Comparison of the morphological type of the members. There is no tendency of the members to share the same morphological type

Pair 129 (AM0608-333). The pair, in the ESO-LV, is composed of $\mathrm{Sbc}+\mathrm{S} 0$ a galaxies (in RC3 both are classified as S?). The members are strongly interacting. The RR95 indicates that 129 is probably part of a group. Only the profile of 129a has been obtained; 129b is very distorted. The colour profile behaves like that of 'normal' spirals and is very blue in the outskirts (see also the colour map). $129 \mathrm{~b}$ is later in type than 0.0 given by the ESO-LV, since arms, probably created/modified by interaction but bluer than the disc, are visible.

Pair 131. The pair consists of two spirals of type later than Sbc. The luminosity profiles in fact are dominated by the exponential disc. The colours are typical of late spirals.
Pair 133 (NGC 2211/12). The ESO-LV classified the pair as an S0+S0. 133a is a classical lenticular as can be seen by the exponential disc. The ellipticity grows monotonically up to 0.6 in the inner $10^{\prime \prime}$ and then stabilizes at this value up to the last observed point. An $X$-structure is present in the central part (see Fig. 4 in Reduzzi et al. 1994 for the image). The $X$-structure is concentric with the outer isophotes since the twist, outside the seeing disc, is negligible. The colour profile is constant with $(B-V) \approx 1.1$, so the colour of the galaxy is not modified by the presence of this structure. 133b is unusually blue, starting from the very centre, for the morphological classification assigned by the ESO-LV. In the isophotal 
map a strong twist is visible probably due to a bar-like structure.

Pair 136 (AM0631-341). The pair is classified as $\mathrm{S}+\mathrm{E} / \mathrm{S} 0$ in the ESO-LV. The luminosity profiles suggest that both galaxies have an exponential disc. 136b has colour profiles redder than a typical early-type galaxy, while those of $136 \mathrm{a}$ are typical of spirals of Sc class. The $a 4 / a$ profile of $136 \mathrm{~b}$ is up to $1.5 \%$ boxy inside $10^{\prime \prime}$, while outside the galaxy shows significantly discy isophotes (up to $2 \%$ ).

Pair 143 (NGC 2305/07) (AM0648-641). The velocity difference of this $\mathrm{E}+\mathrm{S}$ pair, as deduced from the RR95 catalogue, is quite high $\left(1067 \mathrm{~km} \mathrm{~s}^{-1}\right)$. Even if the pair is really isolated and the magnitude difference between members is only $0.56 \mathrm{~B}$-mag this could be an optical pair. 143a is an elliptical with some sub-structures visible both in the ellipticity and in the $a 4 / a$ profiles. It appears in the Knapp et al. (1989) compilation among early-type with IRAS (upper limit) flux $\left(S_{12 \mu}=0 \pm 27, S_{25 \mu}=0 \pm 22\right.$, $\left.S_{60 \mu}=0 \pm 40, S_{100 \mu}=0 \pm 91\right)$. 143b shows a well developed bar and a ring which are frequently found in interacting objects; the arms' structure is grand design. Since both galaxies are very bright, the background could be probably overestimated with the typical cut-off sign in the fainter part of the profile (see 143a). The colour profiles are normally red for 143a and typical of its spiral class for $143 \mathrm{~b}$. In particular the $2 \mathrm{D}$ colour map of $143 \mathrm{~b}$ evidences that the bar has a nearly homogeneous colour $((B-V) \approx 1)$.

Pair 153 (-/IC 2200) (AM0727-621). S0+S pair in the ESO-LV. 153a has a significant isophotal twist (larger than $20^{\circ}$ ) and off-centring in the outer part of the galaxy, in a direction which is not that connecting the galaxy nuclei. The colour is typical of an early-type galaxy (see also the colour map). A model subtracted from the galaxy enhanced a spiral-like feature visible also in the original image (see Fig. 5 in Reduzzi et al. 1994) and the asymmetries are created by this peculiar kind of 'arms'. This kind of features are also visible in a simulation done by Weil \& Hernquist (1993) in a merger remnant. In our case we notice that the asymmetry creates an area, visible in the colour map, redder than the overall disc suggesting that it could be a spatial re-distribution of bulge stars driven by the interaction. This galaxy deserves a spectroscopic study in order to verify the presence of other signs disentangling the possible merging origin. $153 \mathrm{~b}$ is a latetype spiral (Sb-c). The arms are grand design. As visible also in the $2 \mathrm{D}$ colour map the galaxy is quite red also in the outskirts with a $(B-V) \approx 0.7$. RH94 report that the variation among the colour of spirals is quite large. Although the presence of a different amount of dust (and inclination) may contribute to the large dispersion among the spiral classes, this is a good example of the so called 'Holmberg effect' under which paired galaxies with different morphological type tend to have the same colour.
Pair 155. The pair is an Sb-c+Sc in the ESO-LV catalogue. $155 \mathrm{~b}$ is seen nearly edge-on. 155a has a grand design structure, a prominent bar and an inner ring. The $(B-R)$ and the $(B-V)$ colour profiles of 155 a have a different shape. A nearby bright star (just outside the frame) can be responsible for the anomalous behaviour in the $R$-band. The $(B-V)$ colour profile is quite red $((B-V) \approx 0.9)$ for this nearly face-on $\mathrm{Sb}-\mathrm{c}$ galaxy.

Pair 161 (AM0830-593). Sbc+Sa pair in the ESO$\mathrm{LV}$ while $161 \mathrm{~b}$ is classified as uncertain spiral (S?) in RC3. 161a has a bar and the arms are very open and grand design. The colour profiles have the typical trend of objects with the given classification.

Pair 162. S/Irr+Sa pair in the ESO-LV. The objects are visibly interacting as shown by a tail South of $162 \mathrm{~b}$. In both luminosity profiles appear the exponential disc. The galaxies have similar colour profiles although the morphological types are quite different. This could be a possible example of 'Holmberg effect' in action, since the morphological type jumps from 1 to 7 .

Pair 175 (AM1003-435). Sa-b+S/Irr in the ESO-LV classification. Long tails are indicative of the interaction of the two members. It is presumable that the internal rotation of the two galaxies is significant. The luminosity profile is not given because of the strong contamination of one object on the other. Further, the numerous stars in the field and the two particularly bright ones North of $175 \mathrm{~b}$ hinder the interpretation of the $2 \mathrm{D}(B-V)$ colour map. We observe that the $(B-V)$ colour of the Eastern tail is the less contaminated and it is $\approx 0.8-0.9$. The Western tail seems bluer but stars are present. We cannot then infer much from the tails. $175 \mathrm{~b}$ have then the typical colour distribution of a late spiral. We may only suggest that $(B-V)$ colour distribution in the $2 \mathrm{D}$ map of $175 \mathrm{a}$ is reminiscent of a disturbed early-type object rather than a spiral.

Pair 177 (AM1006-380). Sa+E pair in the ESO-LV catalogue while in the RC3 177a is an S0a with outer and inner rings and peculiarities. The presence of a bright star near the pair makes the photometry difficult. 177a shows a strong bar and a distorted ring probably created by the interaction itself. In the $2 \mathrm{D}$ colour map it is possible to distinguish the two rings reported by RC3 which appear slightly bluer than the rest of the galaxy. In the colour profile the internal ring is clearly visible as a sudden change in the trend. $177 \mathrm{~b}$ is an elliptical galaxy. The luminosity profile follows an $r^{1 / 4}$ law, although the contamination of the companion is visible in the outer parts of the profile. The colour is typical of such morphological type. In the outer part the $a 4 / a$ profile becomes significantly boxy up to $1.5-2 \%$, but the contamination of the light by the nearby companion (which is in any case masked during the modelling) could account, in part, for this effect. Outside the region contaminated by the seeing the position angle of the isophotes is nearly constant. 
Table 3. Salient data of pairs from the literature

\begin{tabular}{|c|c|c|c|c|c|c|}
\hline $\begin{array}{l}\text { RR95 Pair } \\
\text { number }\end{array}$ & $\begin{array}{l}\text { ESO-LV ident. } \\
\text { a/b comp. }\end{array}$ & $\begin{array}{c}\text { Projec.sep. } \\
\left({ }^{\prime \prime}\right)\end{array}$ & $\begin{array}{c}\Delta V \\
\left(\mathrm{Km} \mathrm{s}^{-1}\right)\end{array}$ & $\begin{array}{c}\text { Type } \\
\text { a/b comp. }\end{array}$ & $\mathrm{L}_{a} / \mathrm{L}_{b}$ & $a_{25, \mathrm{a}} / a_{25, \mathrm{~b}}$ \\
\hline 24 & $\mathrm{E} 2440120 / 1$ & 18 & 166 & $3.0 /-0.3$ & 0.31 & 0.48 \\
\hline 62 & $\mathrm{E} 5450400 / 410$ & 100 & & $-2.0 / 5.5$ & 8.55 & 1.01 \\
\hline 94 & E1190130/140 & 100 & & $4.0 / 7.0$ & 2.53 & 1.22 \\
\hline 98 & E330040/50 & 86 & & $4.0 / 1.0$ & 1.17 & 1.23 \\
\hline 99 & $\mathrm{E} 5520490 / 500$ & 32 & 86 & $3.0 / 4.0$ & 1.64 & 0.97 \\
\hline 100 & E3610250/3620010 & 173 & 25 & $3.1 / 0.0$ & 2.13 & 1.55 \\
\hline 101 & $\mathrm{E} 4860170 / 190$ & 98 & 130 & $-2.5 /-3.5$ & 0.36 & 0.71 \\
\hline 105 & $\mathrm{E} 4860290 / 300$ & 94 & & $-3.5 / 8.4$ & 7.45 & 0.77 \\
\hline 106 & $\mathrm{E} 4220370 / 390$ & 157 & 34 & $1.0 / 1.0$ & 0.47 & 1.36 \\
\hline 120 & E160090/100 & 510 & & $8.0 / 0.8$ & 0.21 & 0.70 \\
\hline 129 & $\mathrm{E} 3640350 / 360$ & 76 & 193 & $4.0 / 0.0$ & 0.83 & 1.12 \\
\hline 131 & $\mathrm{E} 5560050 / 60$ & 111 & & $4.0 / 6.0$ & 1.20 & 1.70 \\
\hline 133 & $\mathrm{E} 5560130 / 140$ & 99 & & $-2.0 /-1.0$ & 2.07 & 0.99 \\
\hline 136 & E3650280/290 & 84 & & $5.0 /-3.5$ & 1.33 & 1.36 \\
\hline 143 & E870440/450 & 234 & 1067 & $-5.0 / 3.0$ & 1.68 & 1.22 \\
\hline 153 & E1230110/120 & 87 & 73 & $-2.3 / 3.5$ & 1.08 & 1.02 \\
\hline 155 & $\mathrm{E} 880230 / 240$ & 160 & & $4.5 / 6.0$ & 7.25 & 0.76 \\
\hline 161 & E1240180/190 & 106 & 53 & $4.3 / 0.9$ & 1.49 & 2.02 \\
\hline 162 & $\mathrm{E} 5640100 / 110$ & 38 & & $7.0 / 1.0$ & 0.36 & 0.75 \\
\hline 175 & E2630030/31 & 36 & 1 & $1.6 / 7.0$ & 1.12 & 0.89 \\
\hline 177 & E3160320/330 & 59 & 333 & $1.0 /-5.0$ & 1.14 & 1.05 \\
\hline 181 & $\mathrm{E} 5670520 / 530$ & 98 & 106 & $-2.0 /-0.5$ & 2.65 & 0.98 \\
\hline 182 & E3170180/200 & 217 & & $10.0 / 5.3$ & 0.05 & 0.54 \\
\hline 184 & $\mathrm{E} 4360180 / 190$ & 268 & & $-0.2 / 1.0$ & 0.70 & 0.90 \\
\hline 187 & E3750640/650 & 160 & 129 & $1.0 /-1.0$ & 0.41 & 0.57 \\
\hline 197 & $\mathrm{E} 4380200 / 210$ & 40 & 0 & $3.0 / 9.4$ & 2.86 & 1.49 \\
\hline 204 & E3790200/210 & 88 & 518 & $1.0 / 3.0$ & 0.58 & 0.50 \\
\hline 210 & $\mathrm{E} 4400540 / 560$ & 59 & 362 & $-4.0 /-2.0$ & 1.27 & 0.93 \\
\hline 211 & E3210060/70 & 155 & & $4.0 / 1.0$ & 3.84 & 2.02 \\
\hline 215 & E5060010/20 & 539 & 685 & $-3.5 / 4.3$ & 3.37 & 0.66 \\
\hline 216 & E3220040/60 & 118 & 73 & $-0.5 /-3.0$ & 0.28 & - \\
\hline 217 & E3220190/200 & 90 & 185 & $6.0 / 8.0$ & 0.59 & 1.38 \\
\hline 222 & $\mathrm{E} 5070270 / 280$ & 144 & 285 & $-2.0 / 1.0$ & 1.13 & 1.10 \\
\hline 225 & $\mathrm{E} 5070450 / 460$ & 131 & 273 & $-2.0 /-3.5$ & 2.81 & 1.80 \\
\hline 244 & $\mathrm{E} 4440270 / 280$ & 102 & 477 & $-5.0 / 0.0$ & 2.33 & 1.40 \\
\hline 248 & $\mathrm{E} 4440440 / 450$ & 53 & 59 & $3.0 /-5.0$ & 0.97 & 1.82 \\
\hline 250 & E3830140/150 & 63 & 204 & $-2.0 /-2.0$ & 0.30 & 0.65 \\
\hline 267 & $\mathrm{E} 5100580 / 590$ & 126 & 4 & $6.0 / 6.0$ & 0.59 & 0.84 \\
\hline 273 & $\mathrm{E} 2210340 / 341$ & 107 & & $-3.4 /-3.4$ & 0.69 & 0.38 \\
\hline 280 & E5120180/190 & 32 & 48 & $-2.0 / 1.0$ & 1.07 & 1.09 \\
\hline 284 & $\mathrm{E} 5140230 / 240$ & 101 & & $6.0 / 2.6$ & 2.06 & 2.44 \\
\hline 285 & $\mathrm{E} 1370440 / 441$ & 25 & & $-2.0 /-1.6$ & 2.19 & 4.79 \\
\hline 287 & $\mathrm{E} 1380290 / 300$ & 174 & & $-0.7 / 1.9$ & 1.66 & 3.02 \\
\hline 290 & $\mathrm{E} 100010 / 20$ & 35 & 75 & $-2.0 / 10.0$ & 0.48 & 0.47 \\
\hline 292 & E1400430/440 & 257 & 3191 & $2.6 /-4.0$ & 0.26 & 0.57 \\
\hline 293 & E1830170/180 & 70 & & $6.7 / 6.0$ & 0.36 & 1.11 \\
\hline 296 & E1840100/110 & 86 & & $4.0 / 9.5$ & 0.61 & 1.10 \\
\hline 297 & E1040360/370 & 155 & & $-3.0 /-5.0$ & 1.68 & 1.22 \\
\hline 298 & E1040451/50 & 31 & & $0.3 /-3.0$ & 0.43 & 0.65 \\
\hline 307 & E2830190/200 & 140 & & $-1.0 /-4.0$ & 0.47 & 0.74 \\
\hline 317 & $\mathrm{E} 4000290 / 300$ & 63 & 4 & $-2.0 / 3.0$ & 2.09 & 1.06 \\
\hline 322 & $\mathrm{E} 2340590 / 600$ & 269 & 49 & $1.0 / 4.7$ & 1.74 & 1.20 \\
\hline 353 & E2880010/20 & 22 & 287 & $0.2 / 4.1$ & 0.83 & 1.17 \\
\hline
\end{tabular}

Notes: data in Cols. 1 to 4 are taken from RR95. Data in Cols. 5, 6 and 7 are the morphological type and the luminosity and diameter (at $\mu_{B}=25$ ) ratios of the pair members obtained from the ESO-LV. 
Pair 181. S0+S0a in the ESO-LV. Both galaxies show tails (181b) or asymmetries in the isophotal structure (181a). These characteristics are probably due to the ongoing interaction. 181a has a twist of the isophotes of $\approx 20^{\circ}$. A structure, probably a lens, is visible in the ellipticity, $a 4 / a$ (larger than $2 \%$ ) and luminosity profiles. 181b is later in type than the companion but not yet a spiral. The colours of both galaxies are consistent with their classification. The tail of $181 \mathrm{~b}$ is similar in colour $((B-V) \approx 0.8)$ to that of $175 \mathrm{a}$. No evident signs of star formation are present. The pair is possibly one of the objects described in Davoust \& Prugniel (1988: DP88) discussed in the next section. This case is particularly interesting since the pair is quite large in comparison with those previously studied, suggesting that the phenomenon is long-lasting as indicated by the Combes et al. (1995) simulations.

Pair 182. Irr+Sc pair. Only the brighter object, $182 \mathrm{~b}$, of this pair has been studied. The pair is among the most hierarchical in the RR95 catalogue. While $182 \mathrm{~b}$ does not show evident sign of distortion, the companion appears quite asymmetric as described by its classification.

Pair 184 (AM1023-291/2). S0+S pair. 184a is classified in the ESO-LV as a lenticular. The luminosity profile and colours confirm such classification. It is disturbed by an interacting, small satellite which shows asymmetries in the isophotal structure similar to tails. 184b is a face-on spiral whose colour profile has a normal behaviour: red in the bulge part (where also in the 2D map the bar and a lens structure are visible) and becomes bluer in the outskirts.

Pair 187 (NGC -/3289). Sa+S0a pair. 187a is a spiral with very blue colour also in the central part where a bar is present. The galaxy is seen nearly face-on so the internal absorption does not influence significantly the measured colour. This behaviour is different from that of $184 \mathrm{~b}$ although the morphology of the two objects is remarkably similar. The arms' structure is typically grand design as in the $184 \mathrm{~b}$ case. We are probably looking at similar galaxies at different stages of an encounter. This information can come from the structure of the arms, which in the case of $187 \mathrm{a}$ are much more open than in 184b. Noguchi's (1990) simulations show that during an encounter a bar forms and arms, induced by interaction, tend progressively to close around it. If this interpretation is correct, $184 \mathrm{~b}$ is seen at a later stage of its encounter with respect to 187a, and the star formation has had the time to fully develop also in the centre. The gas, in fact, as shown by the same simulations, tends to fall towards the centre. A case similar to $184 \mathrm{~b}$ is seen in $143 \mathrm{~b}$. $187 \mathrm{~b}$ is classified as lenticular but diffuse arms are visible in the frame and the bulge is quite boxy (a bar?). The galaxy is seen at high inclination and the absorption can play a significant role in the measured colour. The overall colour, in fact, is as red as that of an early-type. Obvious morphological signs of interaction are not visible, if we exclude the possibility that arms may have been created by the interaction. They are reminiscent of the 'arms' seen in 210b.

Pair 197 (AM1116-290). Sb+Irr in the ESO-LV catalogue, while the RC3 classifies 197a as an uncertain spiral. 197a, the brighter member, is strongly disturbed and with multiple arms. Open arms (or tails, we do not see, in fact, an underlying disc) connect 197b to the companion. The colour profile of $197 \mathrm{~b}$ becomes bluer going toward the outskirts of the galaxy (see also 2D colour map). Isophotes of the galaxies show also a significant twist (larger than $30^{\circ}$ ), while $a 4 / a$ indicates that the inner isophotes are slightly boxy (a small bar?).

Pair 204 (AM1158-345). Sa+Sb pair, strongly interacting. Open arms starting from a well visible central body (nucleus + bar or lens) in 204a with characteristics similar to the case of $197 \mathrm{~b}$. Also the trend of the colour profile is similar. The 'arms' of 204a remain red. This is better visible in the 2D colour map where the colour of the 'open arms' is approximately the same as that of the main galaxy body. In Noguchi's (1990) simulations open arms develop at the beginning of a disc/disc encounter. Stars and possible gas are in an expansion phase caused by the interaction. The disc of the companion, 204b, which is seen edge-on, is strongly distorted. The $2 \mathrm{D}$ colour map indicates that the latter has quite blue and clumpy areas along the disc.

Pair 210 (NGC 4105/06) (AM1204-292). E+S0 in the ESO-LV. The galaxies cover the frame and the sky determination is quite critical in these cases. We probably slightly overestimated the sky background. The ellipticity and twist profiles of 210 a are quite stable, while the $a 4 / a$ profile is boxy in the centre and discy in the outskirts (where the light contamination by the companion is stronger ); furthermore, it has dust in the centre. 210a appears in the Knapp et al. (1989) compilation among early-type with IRAS (upper limit) flux $\left(S_{12 \mu}=0 \pm 21\right.$, $\left.S_{25 \mu}=0 \pm 36, S_{60 \mu}=270 \pm 40, S_{100 \mu}=740 \pm 135\right)$. The companion 210b is classified as lenticular in the ESO-LV. A diffuse, rounded arm (or tail, we do not clearly see the symmetric arm), possibly produced by the interaction, is clearly visible. The structure of the latter is similar to the arms in $187 \mathrm{~b}$, while the central structure is reminiscent of that visible in 204a. 210b also appears in the Knapp et al. (1989) compilation among early-type with IRAS (upper limit) flux ( $S_{12 \mu}=0 \pm 21, S_{25 \mu}=0 \pm 27, S_{60 \mu}=230 \pm 22$, $S_{100 \mu}=630 \pm 151$ ). The colours of both galaxies are consistent with their morphological classification.

Pair 211 (NGC 4112/-) (AM1204-395). This is an $\mathrm{Sbc}+\mathrm{Sa}$ pair. The proximity to bright stars makes the photometry of 211a difficult. In both galaxies the luminosity profile shows the presence of the exponential disc. $211 \mathrm{~b}$ has an isophotal structure very similar to 100b. The colour profile tends to be bluer in the very centre.

Pair 215 (IC 3152/-). E/S0+Sc pair. 215a is an elliptical with normal colour profile. Outside the seeing disc, 
the isophotes show a significant twist, while their shape does not deviate from the elliptical form. $215 \mathrm{~b}$ is a nearly edge-on spiral galaxy with a prominent dust-lane which defines the galactic plane.

Pair 216 (IC 3290/NGC 4373) (AM1222-392). $\mathrm{S} 0 \mathrm{a}+\mathrm{E} / \mathrm{S} 0$ pair. The inner structure in $216 \mathrm{a}$ is similar to that in 204a but the arms' configuration suggests that it is probably seen in a later stage of the encounter. 216a has a central bar and the colour profile is typical of an early-type. The luminosity profile of $216 \mathrm{~b}$ is fitted by an $r^{1 / 4}$ law so it is rather an elliptical with discy isophotes as evidenced by the $a 4 / a$ parameter. The ellipticity and twist profiles are quite flat outside the region contaminated by the seeing. After a model subtraction, a diffuse filamentary structure centred on the nucleus and extended on the opposite side of the companion is enhanced. Irregularly distributed dark areas are present in the residual, probably indication of a dust presence. The galaxy appears in the Knapp et al. (1989) compilation among early-type with IRAS (upper limit) flux $\left(S_{12 \mu}=0 \pm 25, S_{25 \mu}=0 \pm 28\right.$, $\left.S_{60 \mu}=0 \pm 45, S_{100 \mu}=0 \pm 169\right)$.

Pair 217 (AM1226-402). Sc/Sd+Sd pair in the ESO-LV classification (217b is classified Sdm with a bar in RC3). The exponential disc dominates in both luminosity profiles. 217a has a quite red central region. On the opposite, $217 \mathrm{~b}$ is very blue starting from the very centre. No obvious signs of interaction are visible.

Pair 222 (AM1248-255/4). S0+Sa pair in the classification of ESO-LV, while 222b in RC3 is classified as S0a. Between the two galaxies (Fig. 2) 'clouds' of matter are visible. Their configuration makes the origin unclear, although it may be connected to the ongoing interaction. $222 \mathrm{~b}$ shows a strong bar and an inner ring, visible also in the luminosity profile. The colour profile is stable and typical of galaxies earlier than spiral, although arms are evident. 222a is classified as a lenticular galaxy and a strong disc is, in fact, visible both in the luminosity and in the $a 4 / a$ profiles. The twist is negligible.

Pair 225. S0+E/S0 pair in the ESO-LV classification. The luminosity profiles suggest that both galaxies are better classified as Es. Series of shells and/ripples are present in 225a (we detected 4 different layers). The boxiness of the $a 4 / a$ profiles is due to the presence of the ripples. The twist is not significant. 225b is an elliptical galaxy, up to $2 \%$ boxy in the outskirts. A faint ring (a shell?) in the inner region is enhanced by unsharp masking. The colour of the galaxy tends to be bluer in the outskirts.

Pair 244 (NGC 5124/26) (AM1322-300). E+S0a pair in the ESO-LV classification. The RR95 catalogue indicates this object as a possible quartet (in the ESOLV the other two faint galaxies in the field are not listed). 248a is classified as elliptical but the luminosity profile can be better decomposed adding a disc. The galaxy does not show obvious signs of distortion if we exclude a small warp in the isophotes. The twist is significant $\left(\approx 20^{\circ}\right)$. The $a 4 / a$ profile indicates that the galaxy has discy isophotes (up to $3 \%$ ). The companion, $248 \mathrm{~b}$, shows a strong dust lane along the galactic plane which we see slightly inclined. Both colour profiles tend to become bluer towards the outskirts

Pair 248 (NGC 5152/53) (AM 1325-292). Sb+E pair in the ESO-LV classification. 248a is classified as $\mathrm{Sb}$ but the arms show unusual shape and debris of matter are present. On the other side, the companion does not show obvious signs of interaction. The profiles were not computed because of the reciprocal light contamination. We preferred to plot a $2 \mathrm{D}(B-R)$ colour map which shows that in the outer part 248a has a colour typical of a spiral. The colour of the plume of matter visible North-West of 248a's outskirts is contaminated by the presence of two bright stars in the field.

Pair 250 (NGC -/5193). S0+S0 in the ESO-LV classification, while 250b is classified as E in RC3. The luminosity profiles indicate that $250 \mathrm{~b}$ is an elliptical, while the CCD frame shows that 250a is probably an S0a since it seems to show incipient arms as in the case of $187 \mathrm{~b}$. Both galaxies have discy isophotes, 250a up to $4 \%$ and $250 \mathrm{~b}$ up to $2 \%$. The colour profile of both galaxies is consistent with the classification we sketched above. 250b appears in the Knapp et al. (1989) compilation among early-type with IRAS (upper limit) flux $\left(S_{12 \mu}=0 \pm 26, S_{25 \mu}=0 \pm 40\right.$, $\left.S_{60 \mu}=150 \pm 27, S_{100 \mu}=470 \pm 64\right)$. We expect then the presence of diffuse dust.

Pair 267 (AM1401-243). Both members are classified as Sc in the ESO-LV. 267b shows a grand design structure of the arms, which are open and starting from a bar. The colour 2D profile shows normal behaviour for an Sc with a slightly redder centre (the bar) and blue, patchy outskirts. 267 a has quite asymmetric and off-centred isophotes. The overall appearance of this encounter has some analogy with that described for Pair 129. In particular we note the strong asymmetry of $129 \mathrm{~b}$ and 267a.

Pair 273 (NGC 5516/-) (AM1412-475). No obvious signs of interaction are visible in both objects which are early-type galaxies $(\mathrm{E} / \mathrm{S} 0+\mathrm{E} / \mathrm{S} 0$ in the ESO-LV classification). 273a is quite red in colour. The twist is negligible (lower than $10^{\circ}$ ). The isophotal shape parameter $a 4 / a$ indicates that the isophotes do not significantly deviate from elliptical. The isophotes of the companion, $273 \mathrm{~b}$, are discy up to $4.5 \%$ (see also the increase of the ellipticity at the same distance from the centre).

Pair 280 (AM1440-241). We did not attempt to obtain luminosity profiles and geometrical information since the two galaxies overlap and a bright star's corona influences the light distribution. Only the overall isophotal map is given. 280a is classified as a lenticular galaxy. The unsharp masking technique evidences that $280 \mathrm{~b}$ (Sa in the ESO-LV ) has a strong dust-lane system. 
Pair 284 (AM1529-272). The pair is formed by latetype galaxies (Sc+Sab in the ESO-LV classification). Both objects show asymmetries in the isophotal structures: in both cases they are elongated towards the companion. The colour profiles of 284a show a dip at about $20^{\prime \prime}$.

Pair 285. Both galaxies are classified as lenticulars. The pair is quite hierarchical and there are no obvious signs of interaction. In the North-West direction a dustlane system is visible. From the luminosity profile we may deduce that 285a is better classified as $\mathrm{E}$ since it follows strictly an $r^{1 / 4}$ law. Its isophotes are discy up to $2 \%$. The twist is negligible. $285 \mathrm{~b}$ is embedded into the companion and the photometry is quite difficult owing to the light contamination. Both colour profiles are quite red (the low galactic latitude may play a significant role) and constant along all the galaxy.

Pair 287 (AM1724-622). The 'Sacred Mushroom'. A bridge of matter links the two galaxies. Around the brighter one, 287a, the interaction has developed an external (asymmetric) ring. The 2D colour map shows that this ring is bluer than the 287a disc but the large number of foreground star superimposed makes difficult to draw a definite conclusion. The $(B-V)$ colour of the main body of $287 \mathrm{a}$ is $\approx 0.8$ (see Fig. 3 ) in good agreement with the recent measurements of Wallin et al. (1994). In the same paper is given a series of simulations which suggest that the precursor of 287a is an S0 type galaxy. The azimuthally averaged colour profile at all radii seems no different from that of a normal lenticular. The ESO-LV classifies the companion as an Sa. The 2D colour map confirms this classification. It is also possible to see that the straight bridge that links the two galaxies has a colour similar to that of the outskirts of 287b.

Pair 290 (NGC 6438/-) (AM1806-852). The pair is made by a lenticular and an irregular galaxy, following the ESO-LV classification. The encounter has been disruptive for the late type member. The geometry of the early-type member, 290a, has already been investigated by Rampazzo \& Sulentic (1992: pair \#54 in their compilation). de Mello et al. (1995) found that the dominant population in the nucleus of this galaxy is old and metal deficient $\left(\left[Z / Z_{\odot}\right]_{\max }=-0.5\right)$. However, an important flux fraction at $5870 \AA(\approx 37 \%)$ arises from a young component (age $\leq 510^{8}$ years). 290b has arms with HII regions. Their $(B-V)$ colour is about 0.4-0.5. Although foreground stars in the field enhance the noise, the 2D colour map evidences the distribution of star formation areas. Since the morphological type of $290 \mathrm{~b}$ is very late, it is impossible to draw conclusions about possible SF enhancement due to the interaction.

Pair 292 (AM1840-622). Although we obtained the photometry of this pair we consider it an optical alignment, since its $\Delta V=3191 \mathrm{~km} \mathrm{~s}^{-1}$. It appears in the Journal of Observations but has not been considered in the following analysis.
Pair 293 (-/ IC 4782) (AM 1846-553). Sc+Sc pair. Asymmetries in the isophotes and signs of distortion in both members of the pair. The colour profiles are quite blue in the outskirts. The exponential disc dominates in the luminosity profile of $293 \mathrm{~b}$.

Pair 296 (IC 4817/-) (AM1902-561). Both galaxies are late type. (Sbc/Sc). Both members show distortions. In particular 296b arms appear open, diffuse and asymmetric. The overall morphology is reminiscent of 267b. Exponential discs dominate in both luminosity profiles. The colour profiles are consistent with their morphological type.

Pair 297 (NGC 6734/36) (AM1902-653). This is a $\mathrm{E} / \mathrm{S} 0+\mathrm{E}$ pair in the classification reported in the ESOLV. Luminosity and colour profiles are consistent with this classification. 297a is discy in the inner part. No fine structures are visible. 297b is only slightly discy and, in fact, after the subtraction of the galaxy model the residual is a diffuse, elongated structure which probably is not a regular disc. Other two small galaxies are in the field. For this reason in the AM87 catalogue AM1902-653 is classified as a quartet.

Pair 298 (-/IC 4823) (AM1907-640). The pair is an S0a+E/S0 in the classification given by the ESO-LV. The objects are very close and a distinctive sign of interaction is visible in the effect described by DP88. There are two nuclei or a star near the nucleus of 298a perturbing the colour profile. For both members, outside the centre for 298 a and along all the galaxy for $298 \mathrm{~b}$, the colour profile is consistent with their classification. Outside the area contaminated by the seeing the twist of the isophotes is negligible for both members. $a 4 / a$ profiles are quite irregular. The AM87 classifies 298b as E+jet+condensation.

Pair 307. S0+E pair in the ESO-LV catalogue. 307a shows sign of distortion and a presence of a single arm, probably created by the interaction. The arm has a blue colour $(B-R) \approx 1.0-1.1$. The early-type companion, 307b, seems completely unperturbed. Twist is negligible, while the shape of the isophotes jumps from slightly boxy (1\%) to strongly discy (up to $3 \%$ ).

Pair 317 (IC 5013/-) (AM1947-445). S0+Sb pair in the ESO-LV classification but we do not see arms in 317b. The colour profiles are in fact consistent with an early-type classification for both objects. 317a has discy isophotes (up to $4.5 \%$ ) but the luminosity profile is fitted by a simple $r^{1 / 4}$ law. The twist is negligible, while the isophotes of $317 \mathrm{~b}$ show a twist $\left(30^{\circ}\right)$ which is partly the consequence of the light contamination caused by the proximity of the brighter companion. 317a is an IRAS emitter (Knapp et al. 1989) whose flux is $S_{12 \mu}=0 \pm 31$, $S_{25 \mu}=0 \pm 33, S_{60 \mu}=200 \pm 25, S_{100 \mu}=600 \pm 80$.

Pair $\quad 322 \quad$ (NGC 6935/37) (AM2034521). Sa+Sbc pair. The luminosity profiles indicate that both galaxies have an exponential disc. The colour profile 
Table 4. Early-type members of pairs and their structures

\begin{tabular}{|c|c|c|c|c|c|c|c|c|c|c|}
\hline ESO-LV ident. & $\begin{array}{l}\text { Other } \\
\text { ident. }\end{array}$ & $\begin{array}{c}\alpha \\
(1950)\end{array}$ & $\begin{array}{c}\delta \\
(1950)\end{array}$ & Type & $B_{T}$ & $N_{\text {tot }}$ & 1 & 23 & 45 & 5 Notes \\
\hline E2440121 & & 011556 & -444319 & -0.3 & 14.43 & - & & $\bullet$ & & $24 \mathrm{~b}$ \\
\hline E5450400 & & 023553 & -202255 & -2.0 & 13.83 & 1.6 & & & & $62 \mathrm{a}$ \\
\hline E3620010 & & 050006 & -340607 & 0.0 & 15.08 & 1.0 & & & & 100b Arms \\
\hline E4860170 & & 050105 & -225258 & -2.5 & 14.73 & 2.5 & & & & 01a Disc \\
\hline E4860190 & & 050110 & -225407 & -3.5 & 13.62 & 2.5 & & & & 101b Disc \\
\hline E4860290 & & 050529 & -230755 & -3.5 & 14.59 & 1.0 & & & & $105 \mathrm{a}$ \\
\hline E3640360 & & 060811 & -333740 & 0.0 & 14.39 & 21.5 & & & & $129 \mathrm{~b} \mathrm{Sb} ?$ \\
\hline E5560130 & NGC 2211 & 061618 & -183101 & -2.0 & 13.70 & 0.6 & & $\bullet$ & & 133a Discy outer regions \\
\hline E5560140 & NGC 2212 & 061623 & -182952 & -1.0 & 14.49 & 0.6 & & & & 133b Discy inner regions Bar? \\
\hline E3650290 & & 063153 & -341358 & -3.5 & 14.79 & 1.3 & & & & 136b Boxy inside, Discy outer region \\
\hline $\mathrm{E} 870440$ & NGC 2305 & 064818 & -641254 & -5.0 & 12.82 & 3.5 & & & & 143a Optical pair? \\
\hline E1230110 & & 072730 & -621528 & -2.3 & 13.74 & 2.2 & & & & 153a Twist Spiral arms? \\
\hline E3160330 & & 100659 & -380852 & -5.0 & 13.52 & 4.1 & & & & $177 \mathrm{~b}$ \\
\hline E5670520 & & 101745 & -212634 & -2.0 & 13.98 & 2.5 & & $\bullet$ & & - 181a Strong interaction \\
\hline E5670530 & & 101746 & -212811 & -0.5 & 15.04 & 2.5 & & $\bullet$ & & 181b Strong interaction \\
\hline E4360180 & & 102324 & -291640 & -0.2 & 15.12 & 1.6 & & $\bullet$ & & - 184a Small galaxy superimposed \\
\hline E5680080 & & 102343 & -195847 & -5.0 & 14.65 & 0.3 & & & & $185 \mathrm{a}$ \\
\hline E3750650 & NGC 3289 & 103150 & -350353 & -1.0 & 13.68 & 10.2 & & & & 187b Arms/Sa? \\
\hline E3760090 & & 103942 & -325858 & -2.0 & 13.67 & 0.3 & & & & $190 \mathrm{~b}$ \\
\hline E3790090 & IC 2977 & 115241 & -372501 & -2.0 & 13.28 & 0.3 & & & & $200 \mathrm{a}$ \\
\hline E4400540 & NGC 4105 & 120406 & -292858 & -4.0 & 11.35 & 4.1 & & & $\bullet$ & $210 \mathrm{a}$ \\
\hline E4400560 & NGC 4106 & 120410 & -292923 & -2.0 & 11.61 & 4.1 & & & & 210b Arms/Sa? Bar Twist \\
\hline E5060010 & IC 3152 & 121659 & -255204 & -3.5 & 13.28 & 0.6 & & & $\bullet$ & 215a Twist \\
\hline E3220040 & IC 3290 & 122230 & -392952 & -0.5 & 12.97 & 15.5 & & & & 216a Arms/Sa? \\
\hline E3220060 & NGC 4373 & 122239 & -292858 & -3.0 & 11.59 & 15.5 & & $\bullet$ & & 216b Discy \\
\hline E5070270 & & 124857 & -255041 & -2.0 & 13.51 & 10.0 & & & & 222a Disc \\
\hline E5070450 & & 125253 & -263310 & -2.0 & 12.84 & 3.8 & $\bullet$ & & & $225 \mathrm{a}$ \\
\hline E5070460 & & 125302 & -263216 & -3.5 & 13.96 & 3.5 & $?$ & & & 225b Boxy \\
\hline E4440270 & NGC 5124 & 132202 & -200249 & -5.0 & 13.17 & 4.8 & & & & 244a Disc \\
\hline E4440280 & NGC 5126 & 132206 & -300422 & 0.0 & 14.09 & 4.8 & & & $\bullet$ & $244 \mathrm{~b}$ \\
\hline E4440450 & NGC 5153 & 132506 & -292135 & -5.0 & 13.29 & 5.7 & & & & $248 b$ \\
\hline E3830140 & & 132858 & -325858 & -2.0 & 14.00 & 40.7 & & & & 250a Warped disc \\
\hline E3830150 & NGC 5193 & 132902 & -325840 & -2.0 & 12.69 & 40.7 & & & & 250b Discy \\
\hline E2210340 & NGC 5516 & 141240 & -475258 & -3.4 & 13.01 & 1.3 & & & & 273a Red \\
\hline E2210341 & & 141249 & -475345 & -3.4 & 12.60 & - & & & & 273b Disc \\
\hline E5110310 & & 141630 & -270824 & -2.0 & 14.26 & 5.1 & & & $?$ & - $275 \mathrm{a}$ Uncertain \\
\hline E3860040 & & 143725 & -345435 & -2.0 & 13.74 & 1.0 & $\bullet$ & & $\bullet$ & $278 \mathrm{a}$ \\
\hline E5120180 & & 144041 & -241500 & -2.0 & 12.96 & 1.3 & & & & 280a Blue disc \\
\hline E2740060 & & 151247 & -434948 & -5.0 & 14.53 & 1.3 & & & & $282 \mathrm{~b}$ \\
\hline E1370440 & & 164624 & -614347 & -2.0 & 13.68 & - & & & $\bullet$ & 285a Extended dust lanes \\
\hline E1370441 & & 164626 & -614405 & -1.6 & 15.38 & - & & & & $285 b$ \\
\hline E1380290 & & 172429 & -622418 & -0.7 & 12.76 & 1.3 & & $\bullet$ & & 287a Strong interaction \\
\hline E1000010 & NGC 6438 & 180550 & -852504 & -2.0 & 13.37 & 0.3 & & & & 290a Twist \\
\hline E1400440 & NGC 6673 & 184028 & -622052 & -4.0 & 12.69 & 5.4 & & $?$ & & 292b Uncertain-Optical pair \\
\hline E1040360 & NGC 6734 & 190218 & -653224 & -3.0 & 13.75 & 1.3 & & & & 297a Disc, Twist \\
\hline E1040370 & NGC 6736 & 190235 & -653025 & -5.0 & 14.31 & - & & & & $297 \mathrm{~b}$ \\
\hline E1040450 & IC 4823 & 190730 & -640346 & -3.0 & 14.68 & - & & & & $298 \mathrm{~b}$ \\
\hline E2830190 & & 194747 & -450018 & -1.0 & 14.82 & 0.3 & & & & 307a Arms/Sa? \\
\hline E2830200 & & 194754 & -445819 & -4.0 & 13.99 & 0.3 & & & & 307b Discy \\
\hline E4000290 & IC 5013 & 202520 & -361134 & -2.0 & 12.67 & 1.6 & & & & 317a Warped disc \\
\hline E4000300 & & 202522 & -361236 & -3.0 & 13.47 & - & & & & 317b Boxy \\
\hline E1870230 & IC 5063 & 204812 & -571828 & -0.4 & 12.92 & 0.3 & & & $\bullet$ & $331 \mathrm{a}$ \\
\hline E5330310 & NGC 7284 & 222549 & -250600 & -2.0 & 12.96 & 2.9 & & $\bullet$ & $\bullet$ & 381a Boxy \\
\hline E4710471 & & 235351 & -291803 & -3.0 & 14.69 & - & & & & 409b Boxy, Twist \\
\hline
\end{tabular}

Notes: morphological types, total $B$ magnitudes and the galaxy density parameter $N_{\text {tot }}$ are obtained from ESO-LV. The morphological type given in the notes, when present, is deduced from the inspection of the CCD frame. Columns labelled with numbers are: (1) Ripples, Shells, (2) Tail-Plumes, Jets, (3) X-Structure, (4) Dust, (5) Asymmetries 
of 322 a becomes extremely blue in the outskirts, while it is consistent in values and in the general trend with that of a galaxy morphologically earlier that an Sa, possibly an S0a. 322b shows multiple, open arms starting from a prominent bar, while 322 a has small, central open arms.

Pair 353 (AM2143-464). The pair is in a rich environment. The two galaxies are both late type (S0a+Sbc). Colour and luminosity profiles may be contaminated by the light coming from the companion. Both galaxies are very difficult to model and consequently the contribution of each cannot be realistically subtracted. The 2D colour map confirms the classification of the ESO-LV since the colours are typical of their classes. This pair, although less disturbed, is reminiscent of pair 290, but probably represents an earlier phase of the encounter. The colour of $353 \mathrm{~b}$ is similar to that of $290 \mathrm{~b}$.

\section{Quantitative morphology and photometry}

RR95 found that there is no correlation between the morphological types of the pair members contrary to Karachentsev (1972). We have reclassified the galaxies in our sample on the basis of the CCD material. We found that $25 \%$ of the entire sample of pairs have at least one of the members misclassified. This happens especially among early-type objects, with an average error $\langle T\rangle= \pm 1$. In our sample $13.5 \%$ are pairs in which a 'bona fide' elliptical is associated with a spiral $(\mathrm{E}+\mathrm{S})$, a fraction which is in agreement with the findings by Rampazzo \& Sulentic (1992). E+S0 are also represented in the sample with a similar fraction, but the bulk of the present sample is composed by disc/disc pairs $(\mathrm{S} 0+\mathrm{S}$ or $\mathrm{S}+\mathrm{S})$ with a limited presence of irregular galaxies. Some of them are classified as irregular but they may easily be completely distorted spirals (see e.g. pairs members 290b, 296b).

\subsection{Morphological distortions in early-type pairs}

Among very close and isolated $\mathrm{E}+\mathrm{E}$ pairs, DP88 identify a class of objects showing morphological distortions. In this class the outer isophotes of each galaxy are displaced with respect to the inner ones in opposite sense along a direction nearly perpendicular to the line joining the centres of the two galaxies. The deformation appears symmetric with respect to the centre of the pair establishing with a high confidence that they represent physical binaries. Combes et al. (1995: and references therein) simulations show that this kind of feature is produced during an interpenetrating encounter. The displacement is visible also out of the plane of the encounter and it is long-lasting ( $10^{8}$ years). We looked for this effect in our early-type pairs. The pair 298 and possibly 181 show this effect. $14 \%$ of the RR95 southern catalogue of pairs is made by $\mathrm{E}+\mathrm{E}$. Any definite conclusion about the frequency and duration of the DP88 phenomenon needs a study of a statistically significant set of objects extracted from the catalogue.

\subsection{Fine structure in early-type objects}

Early-type galaxies in pair (54 objects in total) have been analysed with the purpose of evidencing the presence of fine structures like shells/ripples, $X$-structure, tails/jets etc. following the scheme outlined by Schweizer (1992). The structures detected are reported in Table 4 .

Both shells/ripples and $X$-structure are rarely detected $(3.7 \%$ and $1.8 \%$ respectively). A large uncertainty about the frequency of fine structures exists since all samples available in the literature are incomplete. Schweizer (1992) extracted a representative sample of galaxies brighter than $B_{T}=13.5$ but the analysis and the frequency refers to part of them (74 objects out of 145 in total). The observations were conducted using the KPNO $0.9 \mathrm{~m}$ telescope. The higher frequency of shells detected ( $56 \%$ for $\mathrm{E}$ and $32 \%$ for S0s) refers to this sample, which is composed, according to the author, mainly of field galaxies. On the opposite, Malin \& Carter (1983) in their southern search for galaxies with shells, found that the final sample represent $16.5 \%$ of isolated ellipticals in RC2. Furthermore, they suggest that these structures are much rarer in denser environments, like groups and clusters. Our results then suggest that the occurrence of shells/ripples in early-type members of pairs is similar to that found by $\mathrm{Ma}$ lin $\&$ Carter (1983) for dense environments. In Reduzzi et al. (1995) the comparison with a sample composed of isolated (i.e. in low density environment) early-type galaxies is discussed.

Eight objects show clear, well organized dust-lane structures. Six early-type galaxies, namely NGC 2305, NGC 4105, NGC 4106, NGC 4373, IC 5013 and NGC 5193 are detected in the IRAS survey mainly at $60 \mu \mathrm{m}$ and at $100 \mu \mathrm{m}$ (see individual notes). The isophotal shape of these objects is either nearly elliptical or discy. The fraction of IRAS-detected early-types is then very small. Also Rampazzo \& Sulentic (1992) found a similar result studying $22 \mathrm{E}+\mathrm{S}$ pairs. The current interpretation is that FIR luminous objects contain cool interstellar matter $\left(10^{8} M_{\odot}\right.$, Jura 1986) in the form of dust that is reprocessing starlight. Dust is actually detected in the centre of NGC 4105 and seems more diffuse in NGC 4373. No special signs of interaction are evident in the other objects if we exclude NGC 4106 in which bar and diffuse arms seem created by the interaction.

\subsection{Spiral structure in binaries}

Elmegreen \& Elmegreen (1982) developed a 12-division morphological system to classify spiral galaxies according to the regularity of their spiral arm structure. This spiral arm classification system correlates with the presence of density waves, e.g. grand design galaxies tend to contain prominent density wave modes. Using these arm classes they classified some samples of spirals belonging to different environments, among them a binary sample 
derived from Turner (1976). The sample of Elmegreen \& Elmegreen (1982) is incomplete and moreover the Turner (1976) binary galaxy sample has been strongly criticized since it included many pairs belonging to groups and even clusters (White et al. 1983). Elmegreen \& Elmegreen found that 1) the binary membership probably influences the spiral structure in non barred galaxies, 2) the binary galaxies with bars are almost always grand design (93\%) and this fraction is larger than the fraction of grand design galaxies among field spirals. Following the Elmegreen \& Elmegreen (1987) revision we succeeded in classifying 36 spirals (Table 5). Some of the spirals in the global sample are, in fact, seen nearly edge-on, strongly interacting or simply do not fit into the Elmegreen \& Elmegreen classes. Four galaxies, namely 175a/b, 197b, 204a, 248a, 287b are generally classified late-type in the ESO-LV although their 'open arms', interpreted in the framework of Noguchi's (1990) simulations, developed during the interaction. In the case of $280 \mathrm{~b}$ the presence of diffuse dust makes the arm classification difficult.

Among barred spirals 21 out of 22 (corresponding to $95 \%$ ) objects have a grand design structure while among non barred galaxies only 7/14 (50\%) galaxies have a grand design structure. The fractions are consistent with the results of the analysis of Elmegreen \& Elmegreen (1982). In particular the grand design structure is more frequently detected in binary than in field spirals both for barred and not barred. Grand design structure is then probably connected with the binary membership.

\subsection{Colours of interaction features and induced star for- mation}

A fraction of our objects may be interpreted in the framework of the simulations performed by Noguchi (1990 and reference therein). The simulated galaxies, in fact, have similar sizes not different from those in our sample. The selection criteria applied (see RR95), tend, in fact, to avoid strongly hierarchical pairs. Besides, a large fraction of our pairs possesses a disc, with a different gas richness. Noguchi's models follow both the stellar and gaseous components evolution in a disc galaxy during the encounter. In the simulations different 'phases' may be evidenced. Just after the perigalactic passage ( 0.4 disc rotations at the outer edge) completely open arms (integral sign) are created by the passage of the companion. A second phase (between 1 and 1.4 disc rotations) may be individuated when a bar develops and arms start to close. The gas is still dispersed all over the disc but it starts to follow the star configuration. After about 2 disc rotations the arms are completely closed around the bar and form a ring. The gas is now mainly concentrated in the centre and in the ring. The last phase described by the models is when the ring starts to be disrupted by the internal dynamics and the overall appearance of the galaxy is nearly asymmetric (after 4 disc rotations). Also the gas is distributed in
Table 5. Arm classes of the spiral members

\begin{tabular}{|c|c|c|}
\hline RR95 Pair & $\mathrm{AC}$ & Notes \\
\hline $94 \mathrm{a}$ & 12 & bar \\
\hline $94 b$ & 2 & \\
\hline $98 \mathrm{a}$ & 12 & bar \\
\hline $99 \mathrm{a}$ & 5 & bar \\
\hline $99 \mathrm{~b}$ & 5 & bar \\
\hline $100 \mathrm{a}$ & 5 & bar \\
\hline $106 \mathrm{~b}$ & 8 & bar \\
\hline $120 \mathrm{a}$ & 4 & \\
\hline $129 b$ & 5 & bar \\
\hline $129 \mathrm{a}$ & 5 & \\
\hline $131 \mathrm{a}$ & 12 & \\
\hline $131 b$ & 2 & \\
\hline $136 a$ & 12 & \\
\hline $143 \mathrm{~b}$ & 9 & bar \\
\hline $153 \mathrm{~b}$ & 12 & \\
\hline $155 \mathrm{a}$ & 9 & bar \\
\hline $161 \mathrm{a}$ & 12 & bar \\
\hline $162 \mathrm{~b}$ & 5 & \\
\hline $177 \mathrm{a}$ & 8 & bar \\
\hline $182 \mathrm{~b}$ & 9 & \\
\hline $184 \mathrm{~b}$ & 9 & bar \\
\hline $187 a$ & 12 & bar \\
\hline $187 \mathrm{~b}$ & 12 & bar \\
\hline $197 \mathrm{a}$ & 9 & bar \\
\hline $211 \mathrm{a}$ & 2 & \\
\hline $216 a$ & 12 & bar \\
\hline $217 \mathrm{~b}$ & 3 & bar \\
\hline $222 b$ & 8 & bar \\
\hline $267 a$ & 4 & \\
\hline $267 \mathrm{~b}$ & 12 & bar \\
\hline $280 \mathrm{~b}$ & $12 ?$ & \\
\hline $290 \mathrm{~b}$ & 1 & \\
\hline $292 \mathrm{a}$ & 12 & bar \\
\hline $296 \mathrm{a}$ & 2 & \\
\hline $322 b$ & 9 & bar \\
\hline $353 \mathrm{~b}$ & 9 & bar? \\
\hline
\end{tabular}

an asymmetric way with the largest concentration in the centre. 1/4 of the total initial gas mass is gathered within $2 \mathrm{Kpc}$ from the nucleus. In this scenario of 'moderate' interaction the bar develops quite soon and it is long lasting, while the ring develops later and the gas follows the configuration of the ring. We then expect that the ring could be a place where SF might occur. The gas finds a stable configuration for about 2-3 disc rotations and may have the time to form stars.

As noticed before a large fraction of the spirals in our sample shows a bar ( $60 \%$ of those classified in Table 5$)$, which could be a transient product of the interaction in Noguchi's models. The bars present in our sample are in the average quite red $\langle(B-V)\rangle \approx 0.91 \pm 0.14$ compared with the galaxy outskirts $\langle(B-V)\rangle \approx 0.66 \pm 0.17$. 
We detected a total of six rings, namely in 106b, 143b, 155a, 177a, 222b, 287a, while the presence of blue structure in the centre of our objects is rare. The average $\langle(B-V)\rangle$ colour of rings is $0.77 \pm 0.15$. In 177 a the distorted ring appears bluer than the galaxy body as seen in Fig. 4. Asymmetric rings may have a more 'traumatic' origin. In the case of 287a, the 'Sacred Mushroom', the ring origin could be a nearly head-on encounter (Wallin et al. 1994). Our $(B-V)$ and $(B-R)$ colours (Fig. 3) of the main body of the galaxy agree very well with those given by the previous authors, $(B-V)=0.87$ and $(B-R)=1.52$ respectively. The ring present in 287 a appears bluer than the body of the galaxy $((B-V) \approx 0.6)$.

From the observational point of view there is no clear indication that SF in tails is enhanced: most of the tails maintain the colour of the outskirts of the progenitor galaxy. Only in few cases there seem to be knotty blue areas which Schombert et al. (1990) interpreted as "short $\left(10^{7} \mathrm{yr}\right)$, weak bursts". On the basis of Noguchi's (1990) models, integral sign arm configuration are made by the distorted disc material (reminiscent of tails), in the first phase of the encounter. Thus they are expected to preserve the colour of the progenitor. This could be the case of 175a, 197b and 204a. Also the tail of the members of the pair 181 shows the colour of the progenitor. The long elongated 'tails' visible in $24 \mathrm{a}$ and $24 \mathrm{~b}$ may be interpreted as long asymmetric arms matching the colour of the arms of a typical spiral. Blue knots are visible in 100a, 175b (although stars are superimposed) and 287b. In any case the main body of the latter galaxies are gas rich spirals and $\mathrm{SF}$ is already expected to happen in the outskirts. Summarizing, in our sample we have indication neither that $S F$ is going on in tails connected to an early-type galaxy progenitor nor that there is a depletion of the SF due to the interaction.

\section{Conclusions}

The study presents the $B, V, R$ surface photometry of 53 pairs of galaxies extracted from a catalogue of southern pairs (RR95). We use the luminosity profile and the geometry of the isophotes in order to give a quantitative morphological classification of each pair member. We are looking for secular evolutionary effects in the photometric and structural properties of galaxies in pairs. The $2 \mathrm{D}$ colour maps have then been produced in order to study the connection between interaction morphology and sign of induced SF activity.

One of the pairs, namely Pair 292, has $\Delta V=$ $3191 \mathrm{~km} \mathrm{~s}^{-1}$ and is then considered an optical alignment. It has not been used in the general discussion.

Very few objects among early-type galaxies $(-5 \leq T \leq$ 0 ) show peculiarities. Approximately $30 \%$ show fine structures. Shells are rarely observed in our sample $(3.7 \%)$. As previously suggested by Malin \& Carter (1983) earlytype galaxies in high density environments show a lower frequency of the shell phenomenon compared to isolated samples.

Six early-type galaxies in our sample are detected by IRAS. Half of them show clear dust structures.

Two pairs seem to show the effect described by DP88. The study of the kinematics of these objects would give us information about the presence of a U-shape rotation curve foreseen by simulations (Borne 1990; Combes et al. $1995)$ and expected from the ablation of the galaxy outskirts.

101a and 133b show in the central part an anomalous blue colour possibly indicating star formation areas.

Arms of spiral galaxies in pairs are of grand design type in $95 \%$ of cases when a bar is present, while $50 \%$ of non barred galaxies are grand design. These results are consistent with findings of Elmegreen \& Elmegreen (1982). In both cases (barred and non barred) spirals in pairs have grand design arms in a larger fraction with respect to their field counterparts, indicating a correlation between pair membership and grand design structure.

We analysed the loci of SF in a selected subsample made of galaxies in the framework of the simulations performed by Noguchi (1990). In this context we observe that among the studied objects the SF in the bar is not modified during the encounter. At the same time the ongoing $\mathrm{SF}$ is not depleted in the outskirts during the encounter. A possible variation in SF rate is detected in the ring which can develop during definite phases of an encounter. Simulations show that the gas tends to collect in a ring while the larger fraction collapses towards the centre. In our sample, the rings tend to be bluer than the nearby parts of the galaxy, i.e. the stellar disc as a whole. This is not only true for late-type galaxies but also for some galaxies which are presumed to be early-type like 177a and 287a, the 'Sacred Mushroom'. It is clear that the SF efficiency is correlated to the quantity of gas present which may be parameterized as a function of the morphological type. At the same time, the gas may also be compressed during an encounter. Combes et al. (1994), in fact, studying CO in pairs show that there is a correlation between the interaction class and the $\mathrm{CO}$ emission. Tidal tails tend to show the colour of the galaxy outskirts, consistenly with the stripping hypothesis. We have no clear indication that $\mathrm{SF}$ can be induced/stimulated in them by the encounter, at the same time $\mathrm{SF}$ is not suppressed when it is active in the progenitor.

In general, our data suggest that a moderate interaction has the function to constrain the gas into certain areas basically the nucleus and the ring. As noticed by Moles et al. (1995) the galaxies produce a "global, well organized response" to the nonaxisymmetric potential created by the interactions. This is especially evident for gas poor galaxies which may develop SF in those areas. The formation of an (asymmetric) ring can be also the response to a more disruptive encounter (Marston \& Appleton 1995) as in the 
case of the 'Sacred Mushroom' in this work (see also simulations of Wallin \& Struck-Marcell 1994). The frequent presence of bars, a transient phenomenon following simulations, indicates that this is the most used mechanism to transfer the gas to the centre and possibly activate both $\mathrm{SF}$ and nuclear phenomena.

Acknowledgements. This paper has benefited from corrections and useful suggestions of the referee Dr. E. Davoust. LR acknowledges the kind hospitality and the use of the facilities of the Osservatorio Astronomico di Brera. The tables of the photometric and geometrical profiles may be requested to LR. We thank Dr. Francesca Rampazzi for the patient debugging of our English.

\section{References}

Arp H.C., Madore B.F., 1987, A Catalogue of Southern Peculiar Galaxies and Associations. Cambridge Univ. Press: AM87

Balcells M., Peletier R.F., 1994, AJ 107, 135

Barnes J.E., Hernquist L.E., 1992, ARA\&A 30, 705

Bender R., Surma P., Döbereiner S., Möllenhoff C., Madejsky R., 1989, A\&A 217, 36

Binney J., Petrou M., 1985, MNRAS 214, 449

Boroson T., 1981, ApJS 46, 177

Borne K., 1990, Dynamics and Interaction of galaxies. In: Wielen R. (ed.). Springer-Verlag, 196

Bushouse H.A., 1987, ApJ 320, 49

Capaccioli M., Caon N., Rampazzo R., 1990, MNRAS 242, 24

Combes F., Prugniel P., Rampazzo R., Sulentic J.W., 1994, A\&A 281, 725

Combes F., Rampazzo R., Bonfanti P.P., Prugniel P., Sulentic J.W., 1995, A\&A 297, 37

Dahari O., De Robertis M.M., 1988, ApJ 331, 727

Davoust E., Prugniel P., 1988, A\&A 201, L30: DP88

de Vaucouleurs G, de Vaucouleurs A., Corwin H.G., et al., 1991, The Third Catalogue of Bright Galaxies. New-York: Springer-Verlag

Elmegreen D.M., Elmegreen B.G., 1982, MNRAS 201, 1021

Elmegreen D.M., Elmegeen B.G., 1987, ApJ 314, 3

Forbes D.A., Thomson R.C., 1992, MNRAS 254, 723

Goudfrooij P., 1994, Ph. D Thesis, University of Amsterdam

Governato F., Reduzzi L., Rampazzo R., MNRAS 261, 379

Heckman T.M., Beckwith S., Blitz L., Skuritskie M., Wilson A.S., 1986, ApJ 305, 157

Hernquist L., Quinn P.J., 1989, ApJ 342, 1

Jura M., 1986, ApJ 306, 483

Kennicutt R.C., 1990, in Paired and Interacting Galaxies,
I.A.U. Colloquium No. 124. In: Sulentic J.W., Keel W.C. and Telesco C.M. (eds.), 269

Karachentsev I.D., 1972, in Catalogue of Isolated Pairs of Galaxies in the Northern Hemisphere, Comm. Spec. Ap. Obs. 7,1

Knapp G.R., Guhathakurta P., Kim D.-W, Jura M., 1989, ApJS 70, 329

Larson R.B., Tinsley B., 1978, ApJ 219, 46

Lauberts A., Valentijn E., 1989, The Surface Photometry Catalogue of the ESO-Uppsal Galaxies, ESO, Garching bei München: ESO-LV

Malin D.F., Carter D., 1983, ApJ 274, 534

Marston A.P., Appleton P.N., 1995, AJ 109, 1002

Mihos J.C., Richstone D.O., Bothun G.D., 1991, ApJ 377, 72

Moles M., Márquez I., Perez E., 1995, ApJ 438, 604

Noguchi M., Ishibashi S., 1986, MNRAS 219, 305

Noguchi M., 1990, in Dynamics and Interaction of galaxies. In: Wielen R. (ed.). Springer-Verlag, 469

Olson K.M., Kwan J., 1990a, ApJ 349, 480

Olson K.M., Kwan J., 1990b, ApJ 361, 426

Pogge R.W., Eskridge P.B., 1993, AJ 106, 1405

Rampazzo R., Sulentic J.W., 1992, A\&A 259, 43

Rampazzo R., Reduzzi L., Sulentic J.W., Medejsky 1995, A\&AS 110, 131

Reduzzi L., Rampazzo R., Sulentic J.W., Prugniel P., 1994, The Messenger 75, 28

Reduzzi L., Rampazzo R., 1995, A. Let. Com. 30, Nos. 1-6, 1: RR95

Reduzzi L., Longhetti M., Rampazzo R., 1995, MNRAS (submitted)

Roberts M.S., Haynes M.P., 1994, A\&A Ann. Rev. 32, 115: RH94

Schombert J.M., Wallin J.F., Struck-Marcell C., 1990, AJ 99, 497

Schweizer F., 1992, Structure, Dynamics and Chemical Evolution of Elliptical Galaxies. In: Danziger I.J., Zeilinger W.W. and Kjar K. (eds.), IPC-ESO Workshop, 651

Smith E.P., Hintzen P., 1991, AJ 101, 410

Stiavelli M., Londrillo P., Messina A., 1991, MNRAS 251, 57

Toomre A., Toomre J., 1972, ApJ 178, 623

Turner E.L., 1976, ApJ 208, 20

Veron-Cetty, M.P., Veron P., 1991, A Catalogue of Quasars and Active Nuclei, ESO Scientific Report 10, 5th ed., Garching ESO

Wallin J.F., Struck-Marcell C. 1994, ApJ 433, 631

Weil M.L., Hernquist L., 1993, ApJ 405, 142

White S.D.M., Huchra J., Lataham D., Davies M., 1983, MNRAS 203, 701 

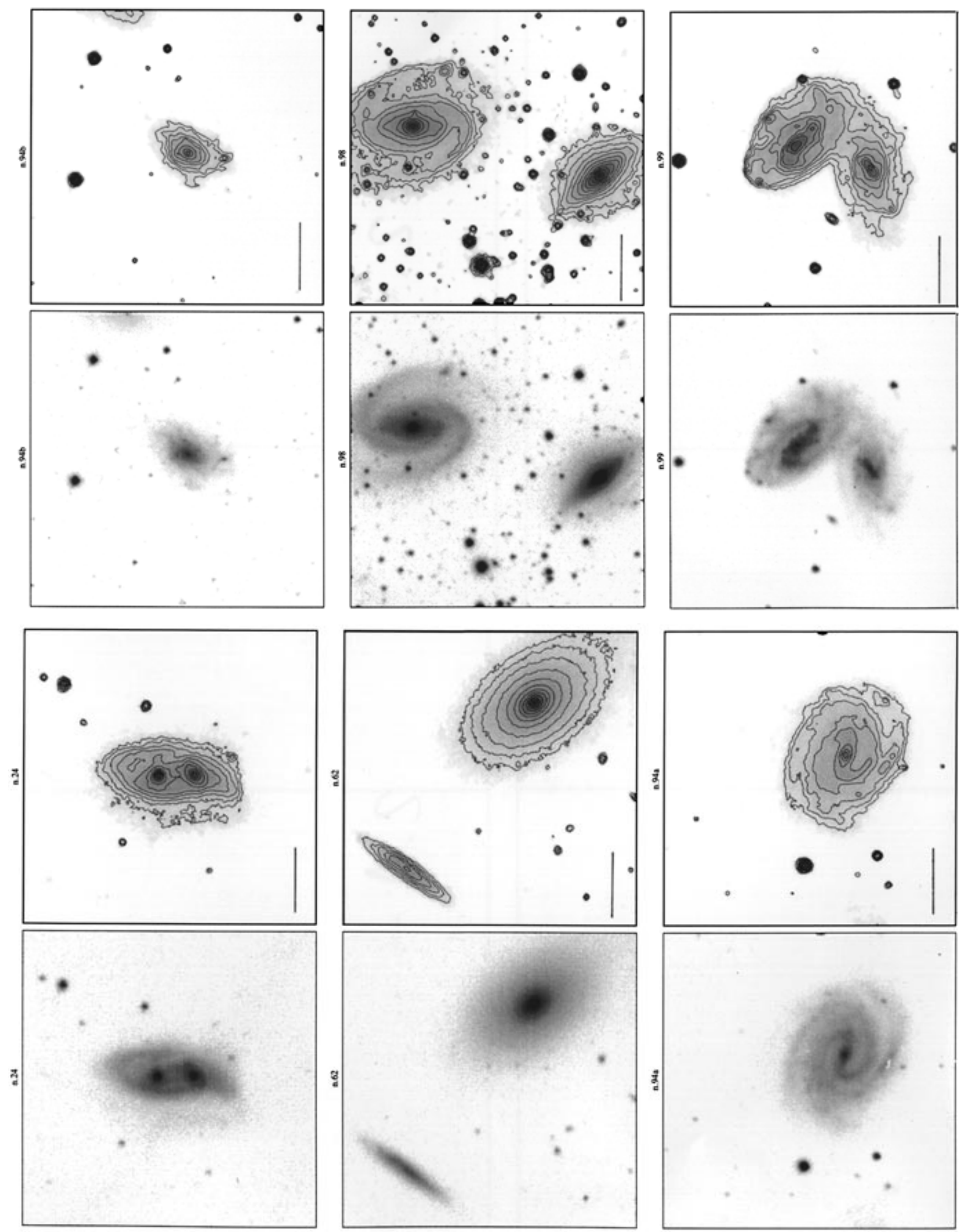

Fig. 2. Images of pairs in $R$ band. The bar, 30 arcsec wide, indicates the scale of the frame (North is at the top, East at the left). The step of the isophotes is $0.5 \mathrm{mag} / \mathrm{arcsec}^{2}$; the external isophote is at $23 \mathrm{mag} / \operatorname{arcsec}^{2}$ while the last grey level reaches $24 \mathrm{mag} / \operatorname{arcsec}^{2}$. (To be seen in landscape) 

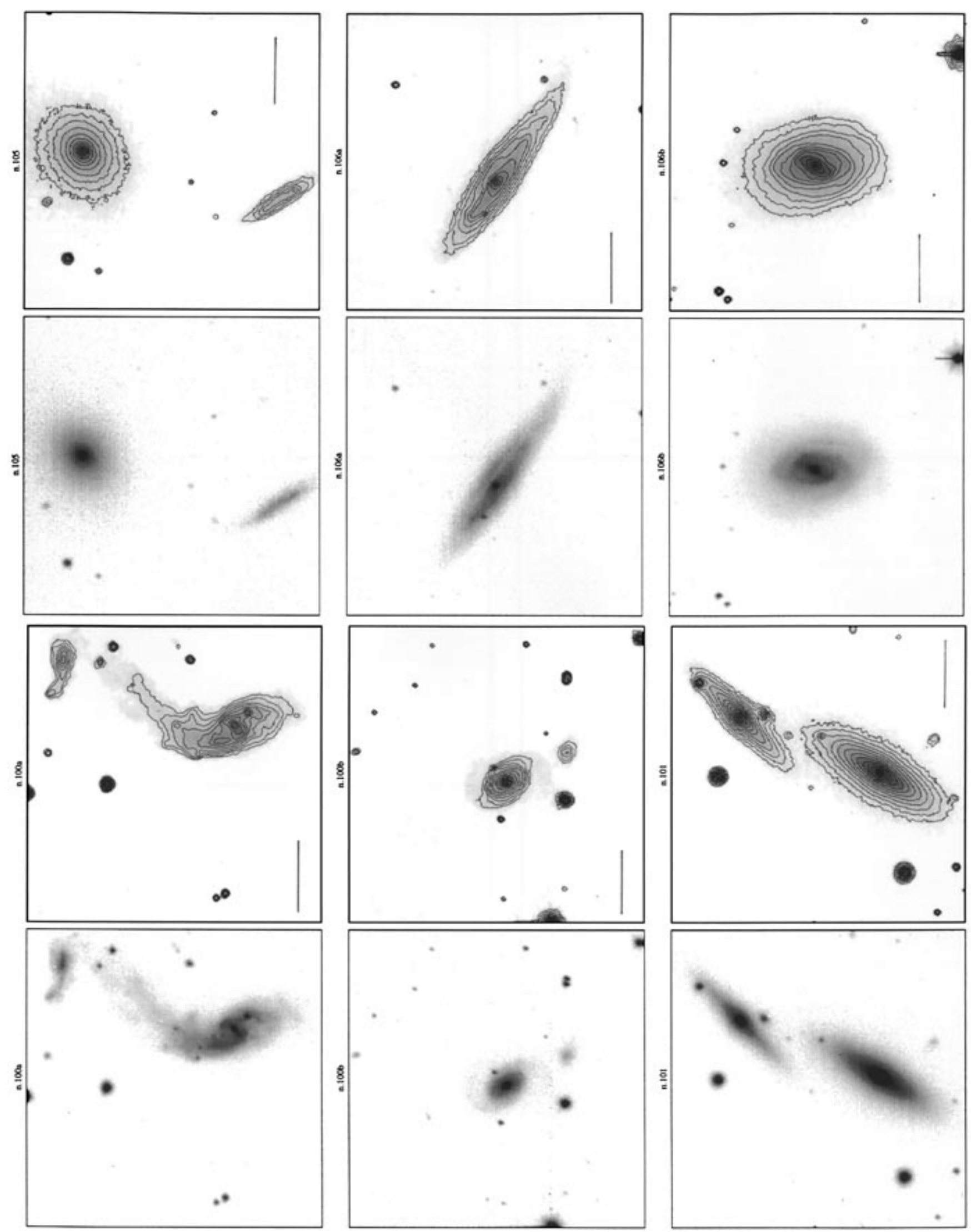

Fig. 2. continued. (To be seen in landscape) 

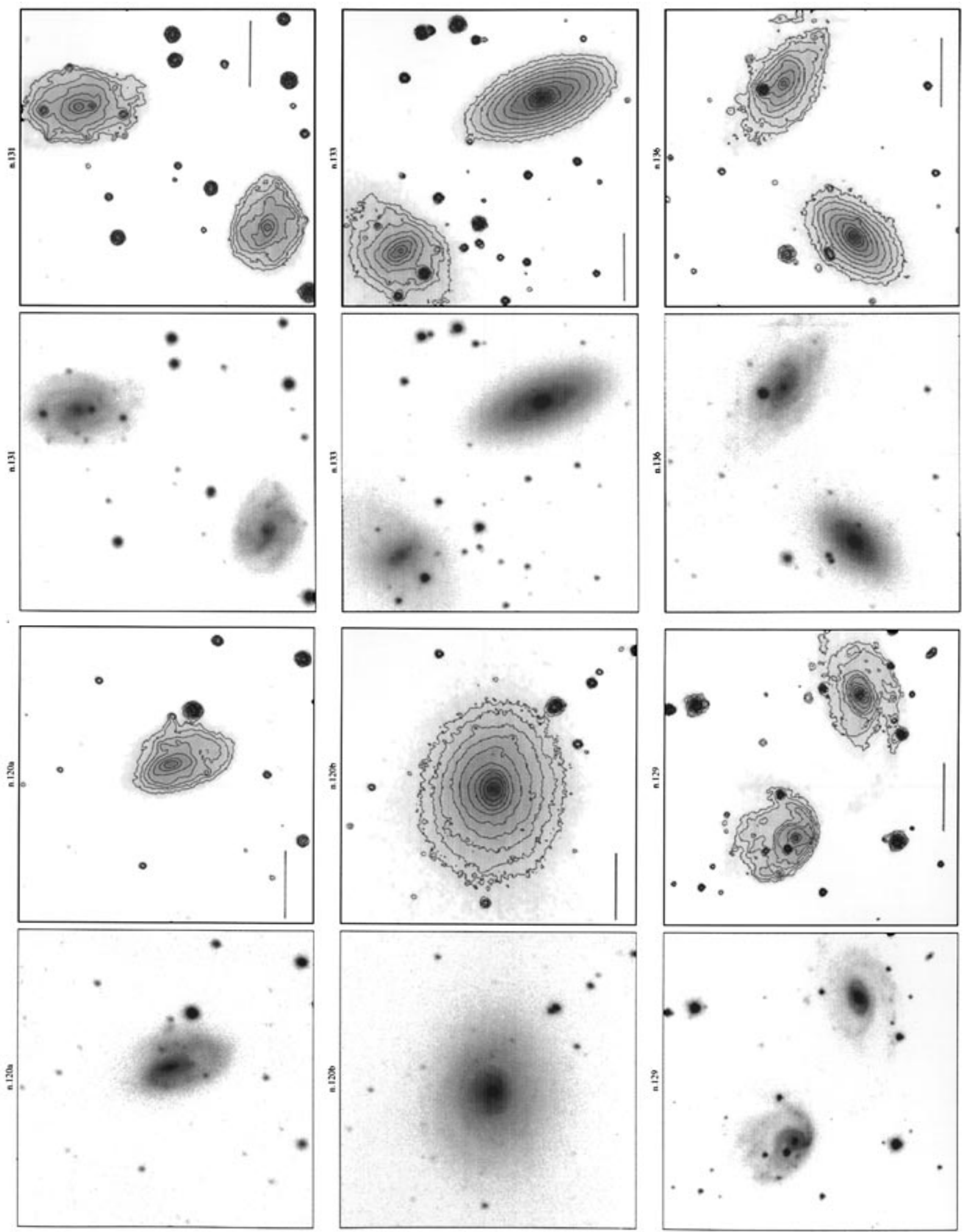

Fig. 2. continued. (To be seen in landscape) 

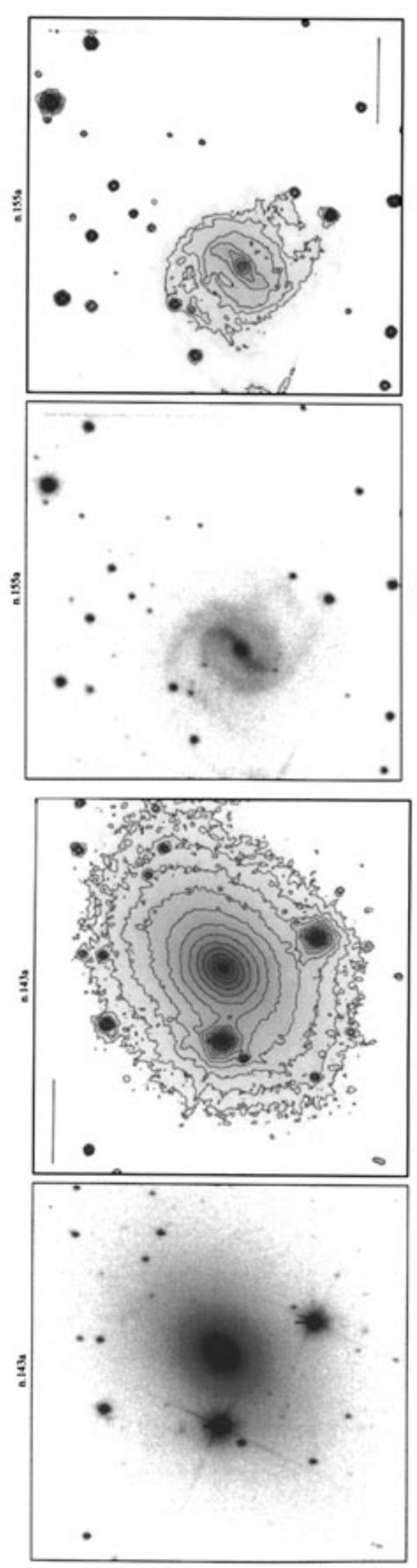
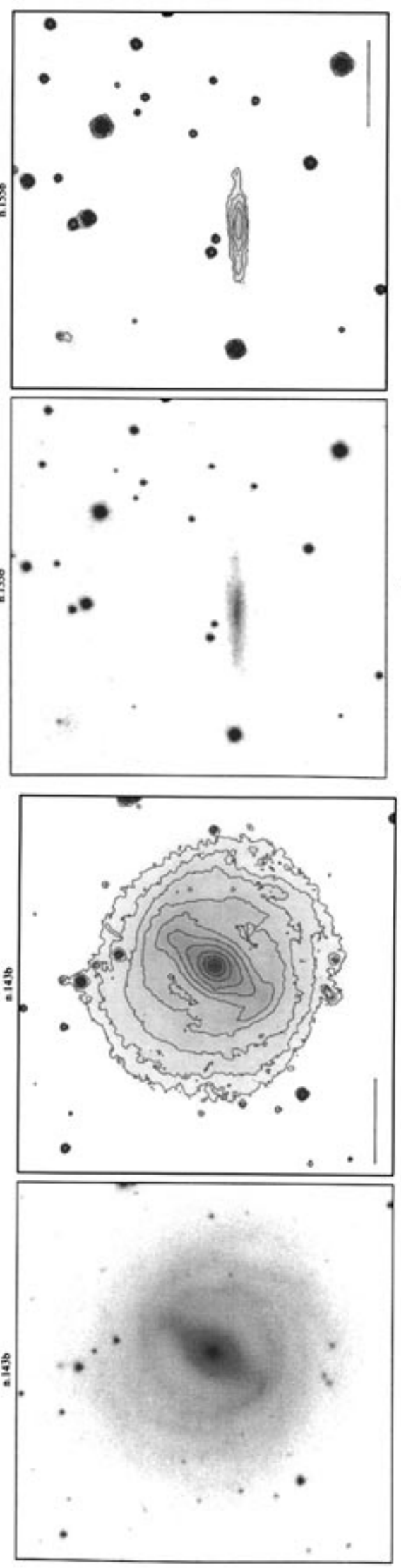
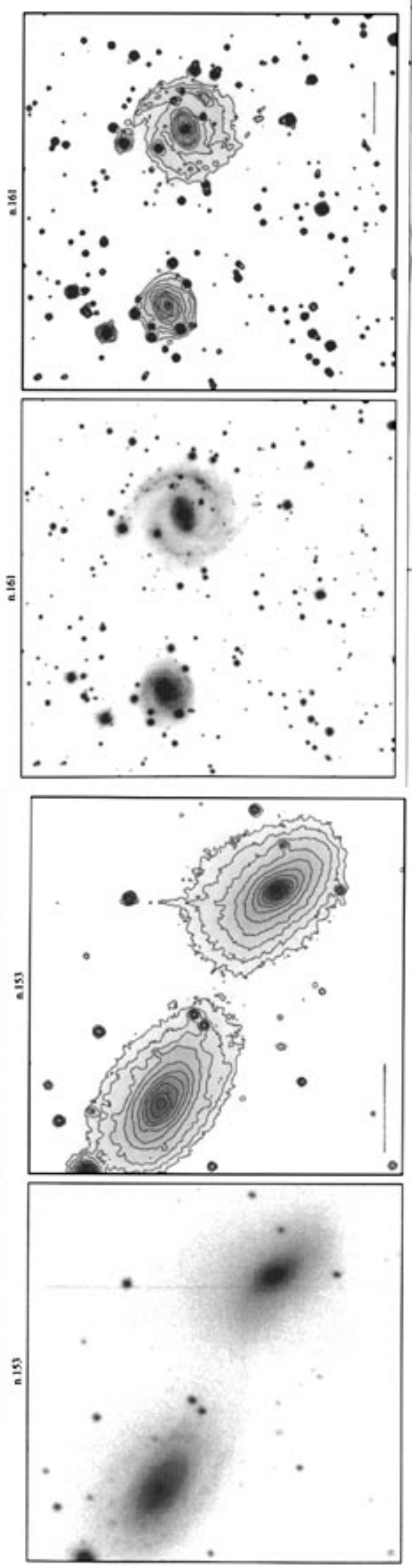

Fig. 2. continued. (To be seen in landscape) 

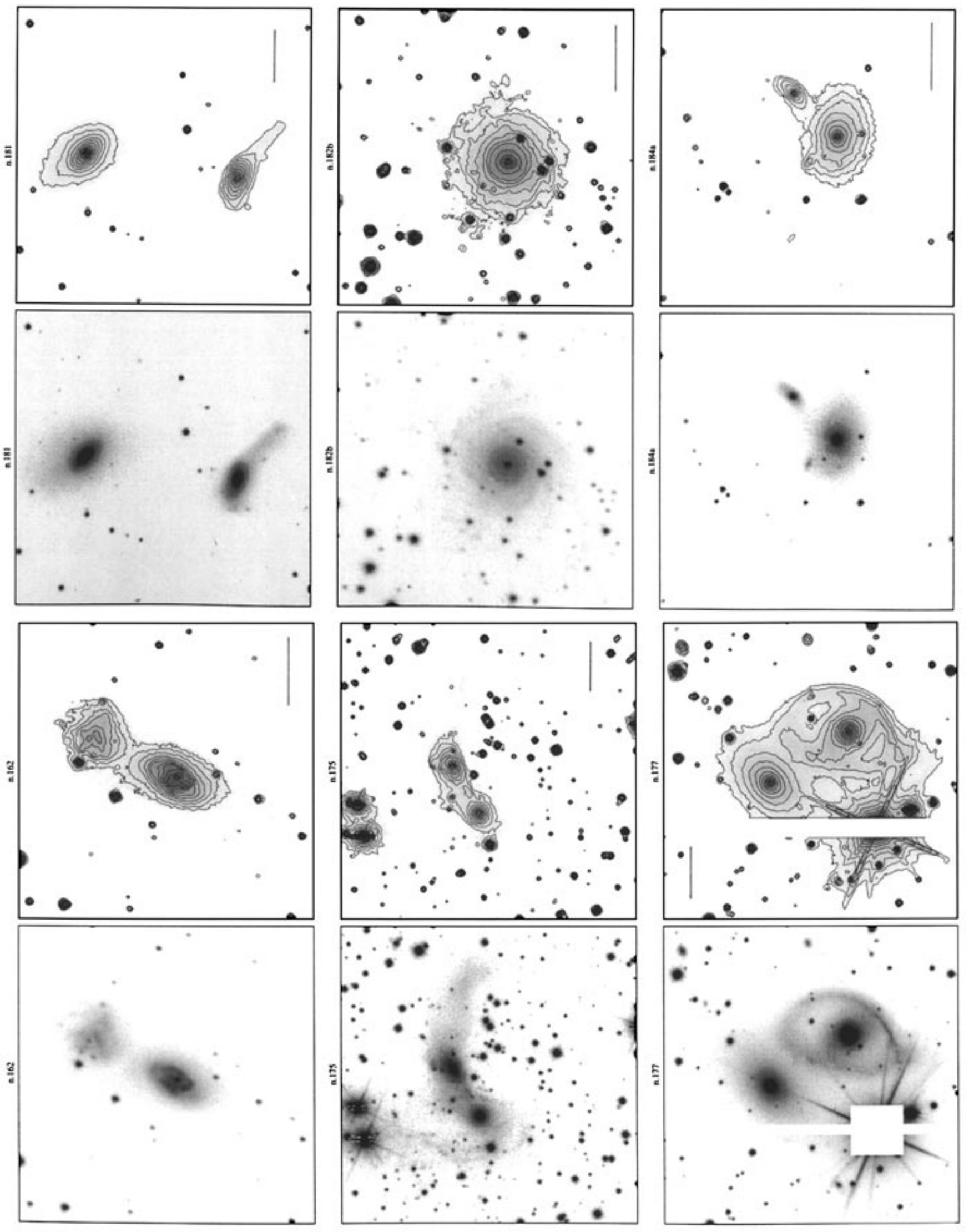

Fig. 2. continued. (To be seen in landscape) 

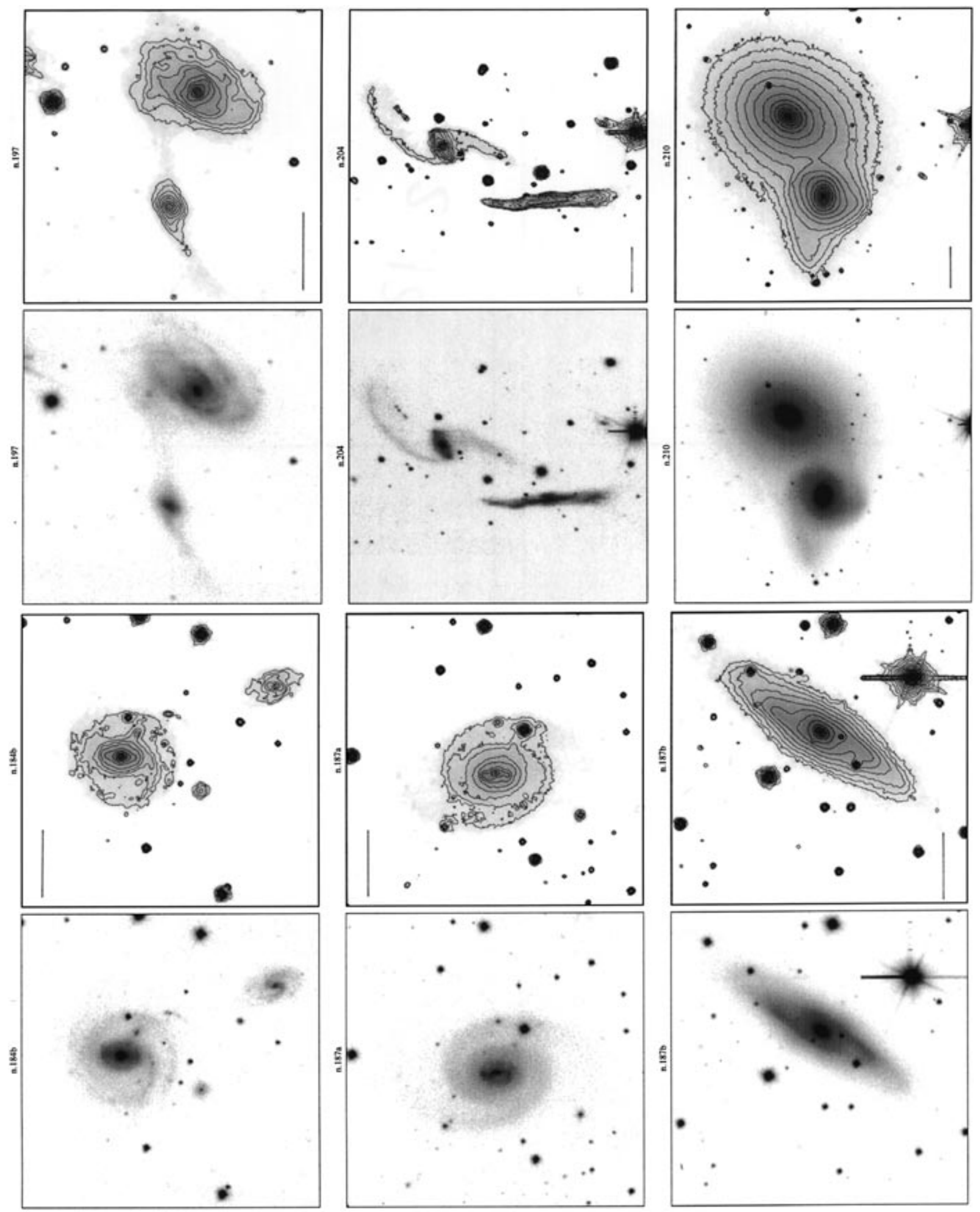

Fig. 2. continued. (To be seen in landscape) 

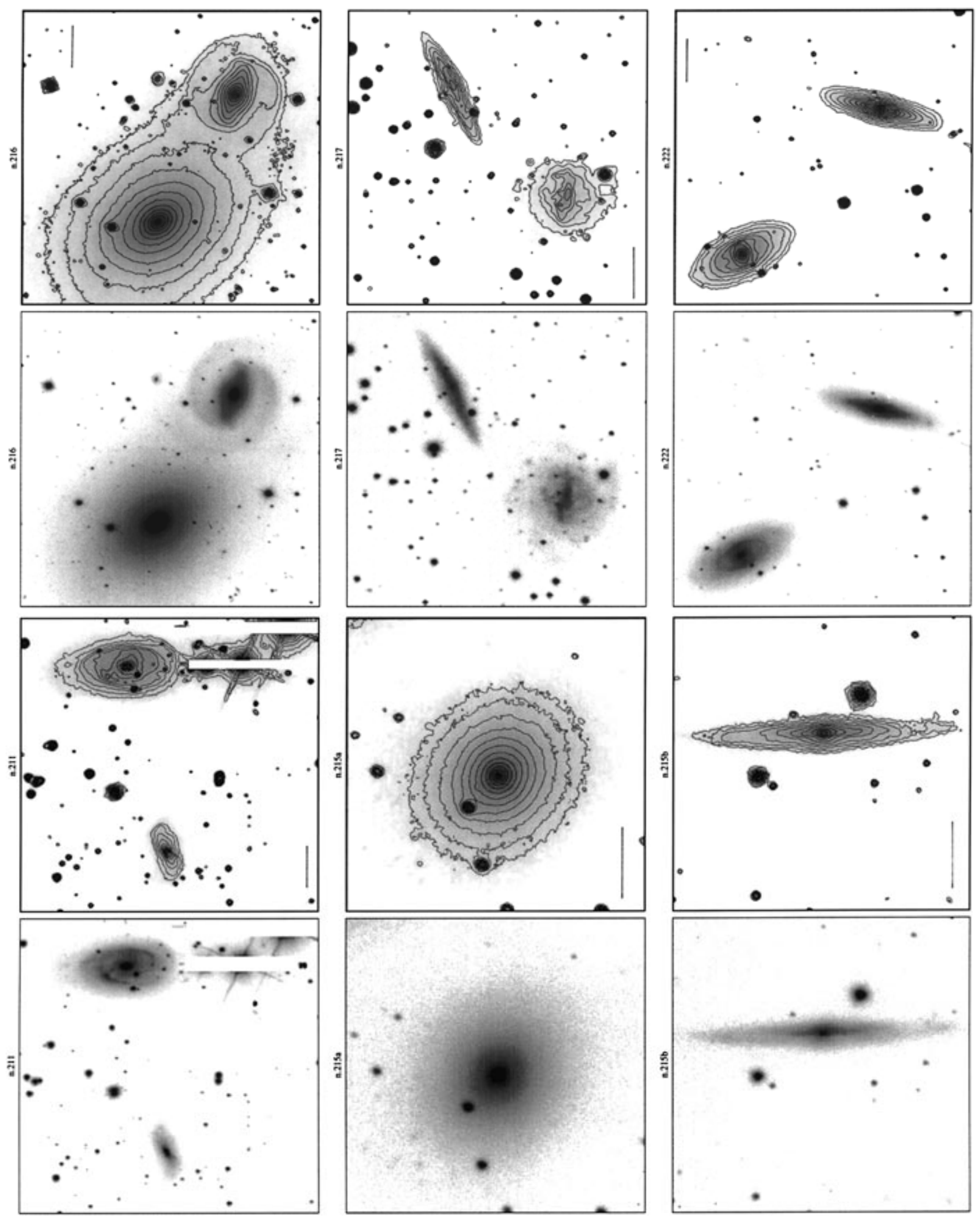

Fig. 2. continued. (To be seen in landscape) 

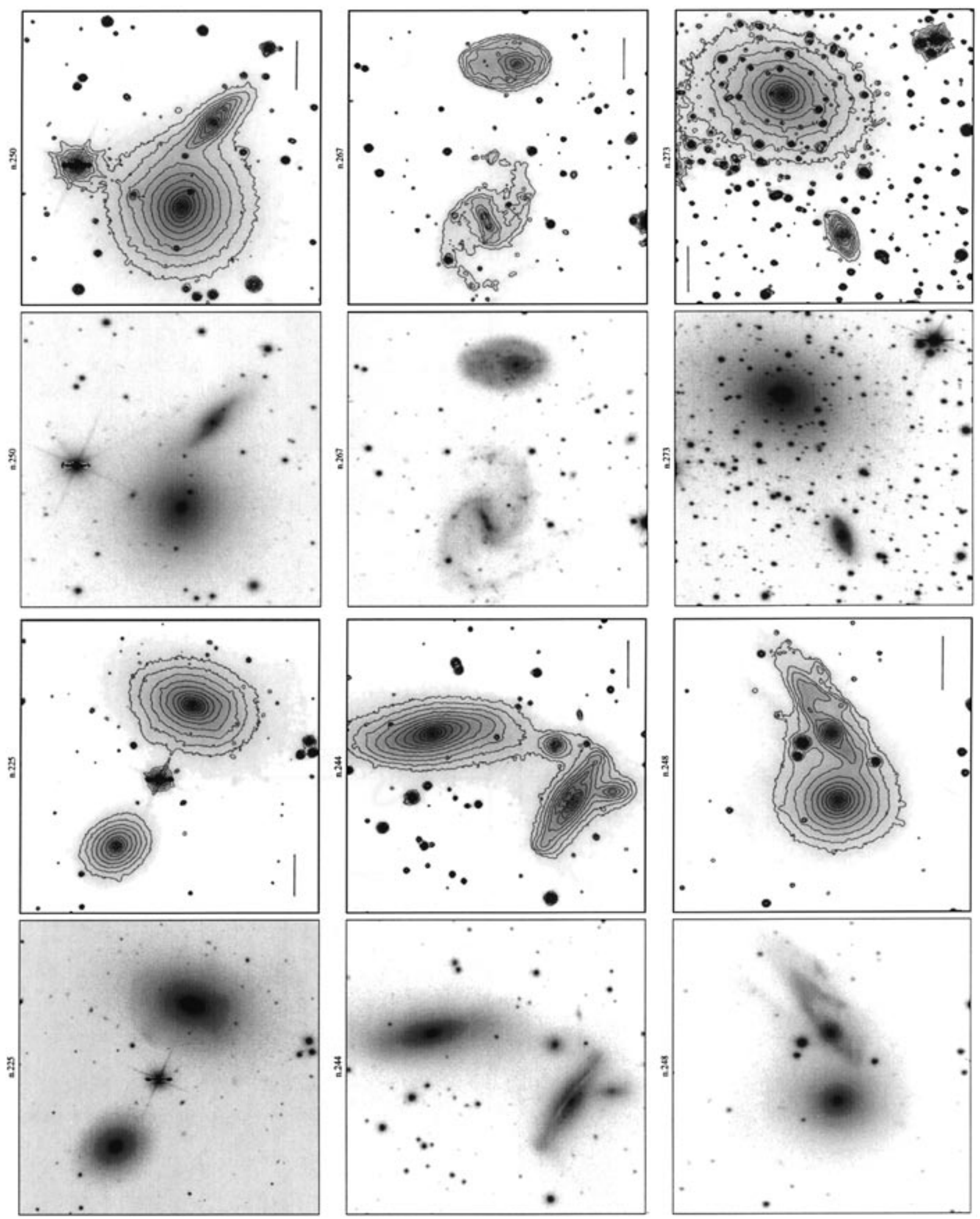

Fig. 2. continued. (To be seen in landscape) 

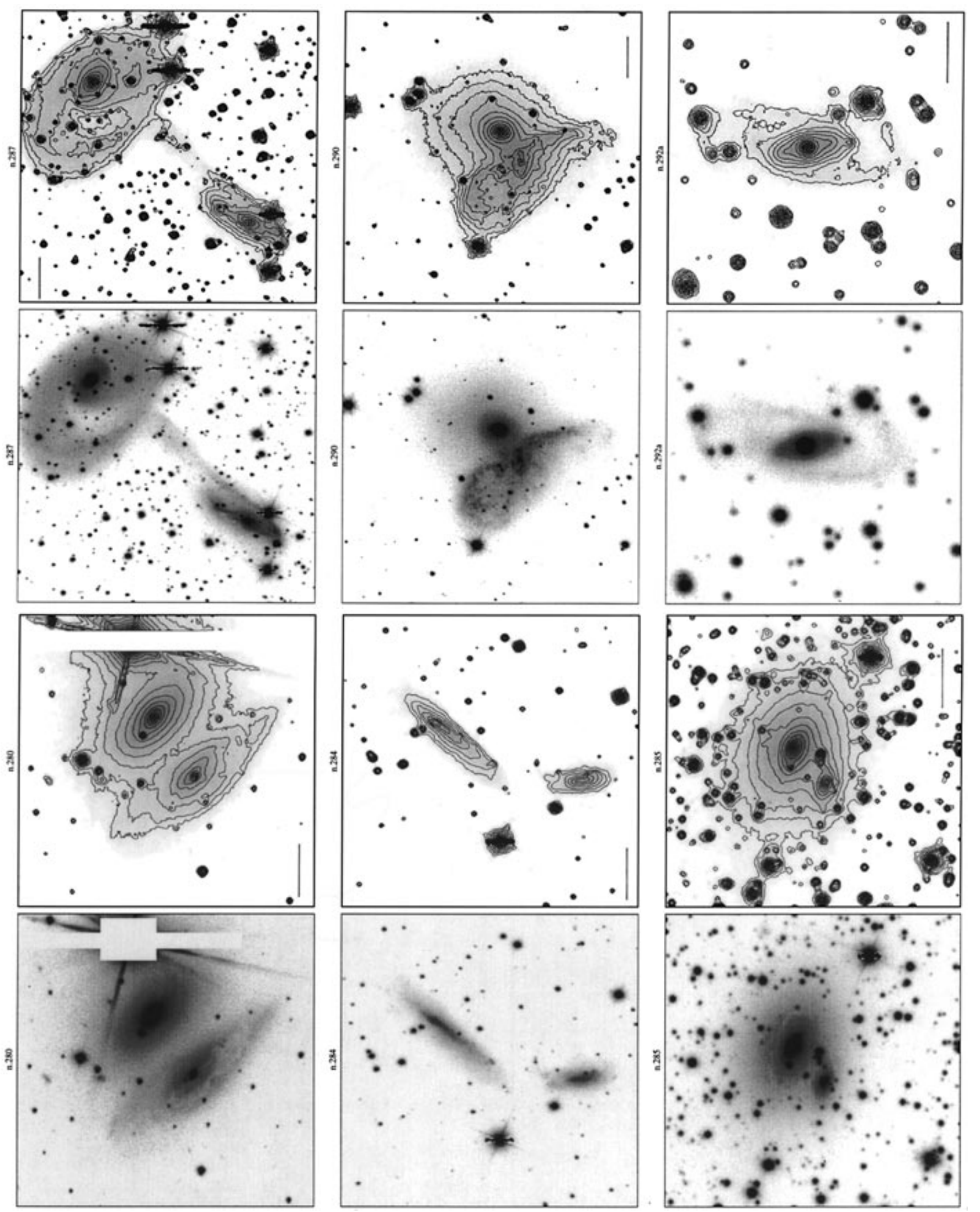

Fig. 2. continued. (To be seen in landscape) 

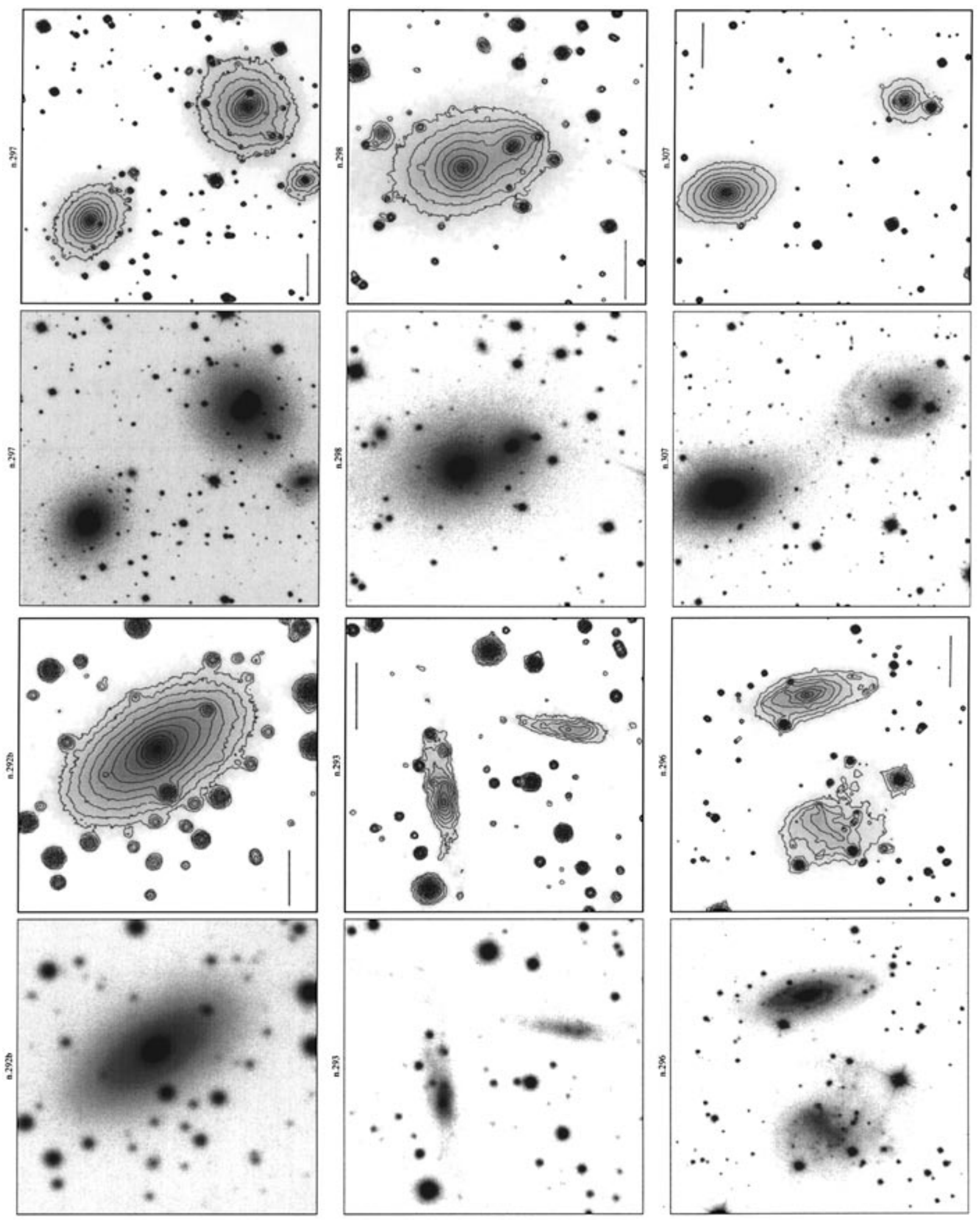

Fig. 2. continued. (To be seen in landscape) 

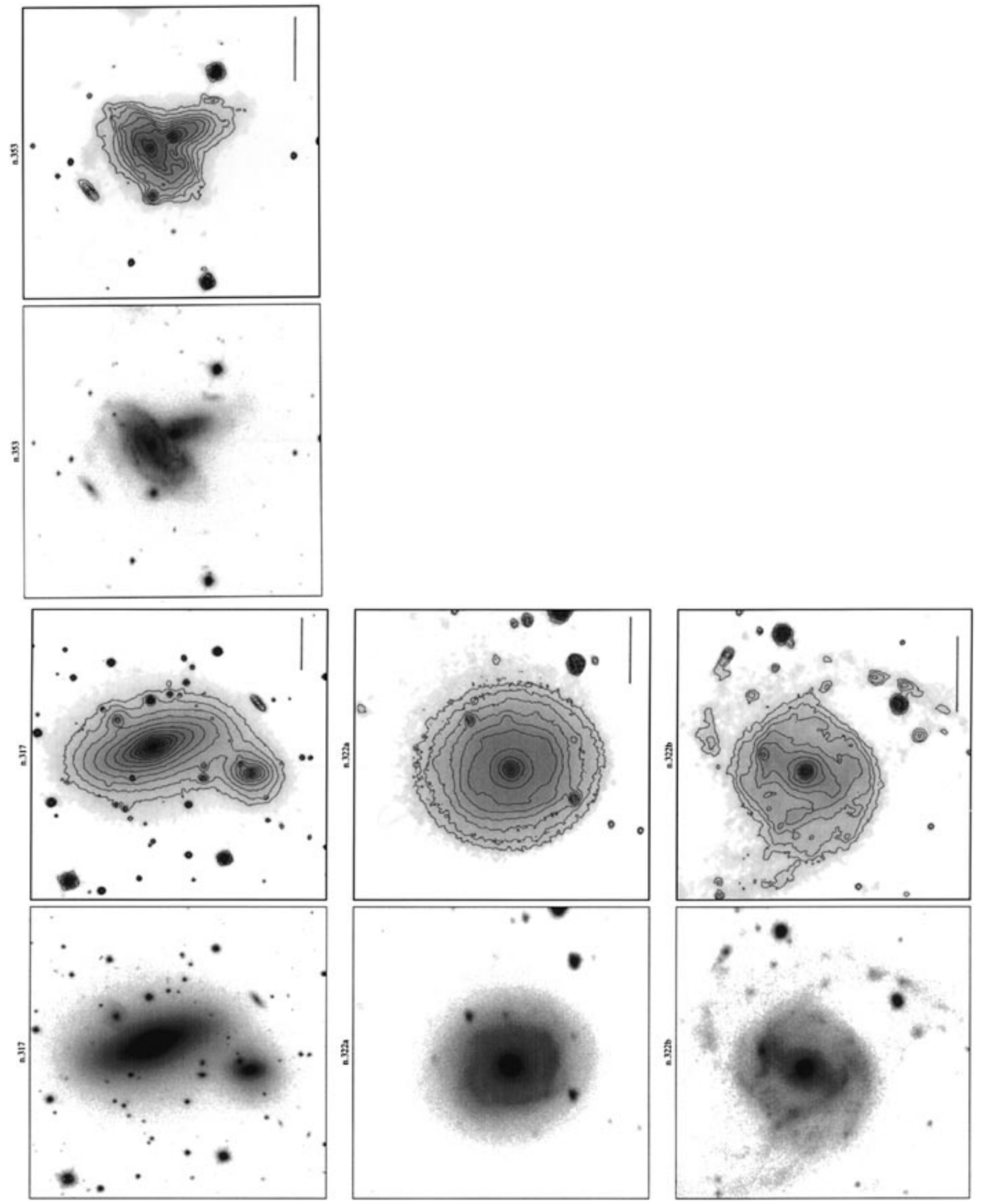

Fig. 2. continued. (To be seen in landscape) 

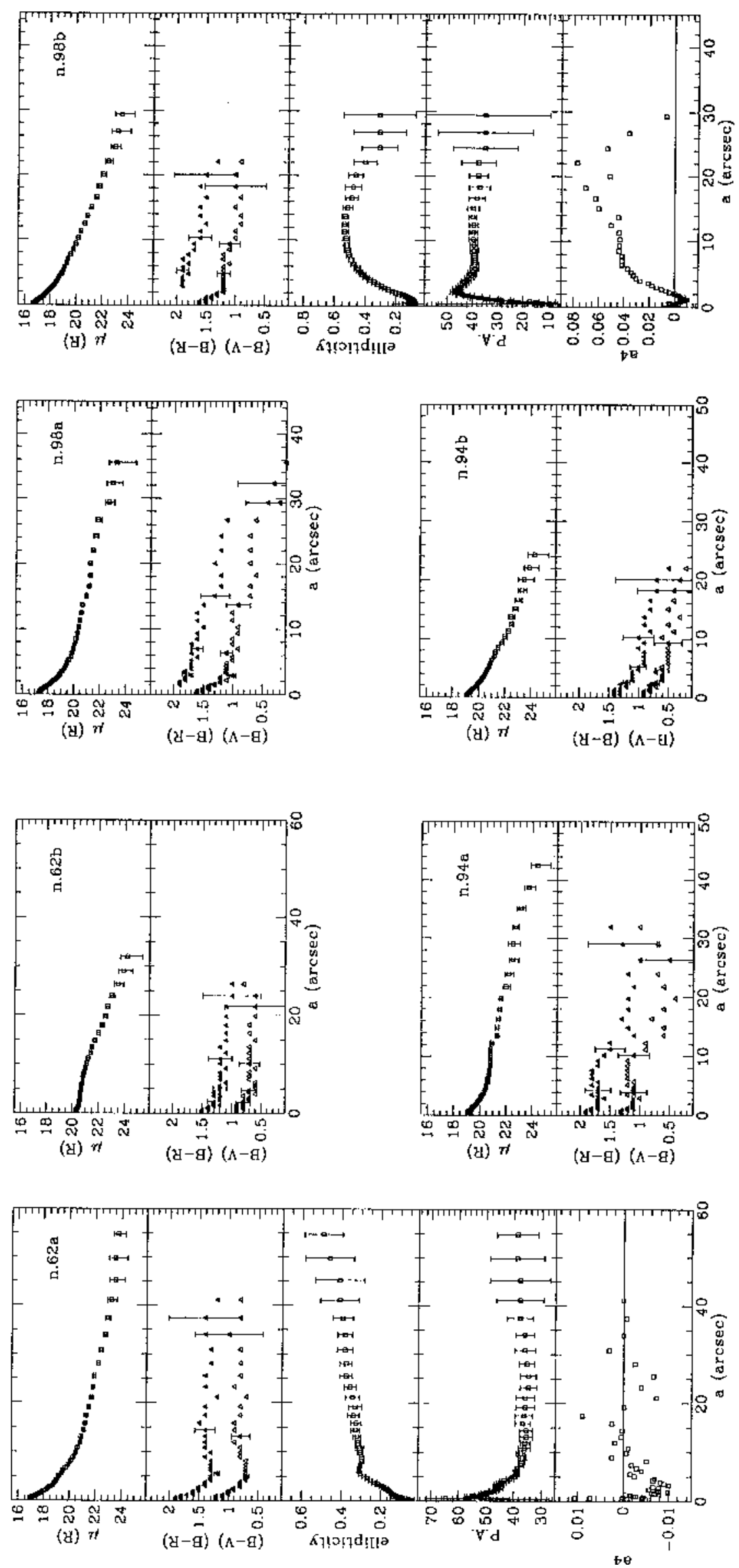

Fig. 3. Luminosity and colour profiles of the members of pairs. In the case of early-type galaxies the ellipticity, twist and isophotal shape profiles are also given. (To be seen in landscape) 

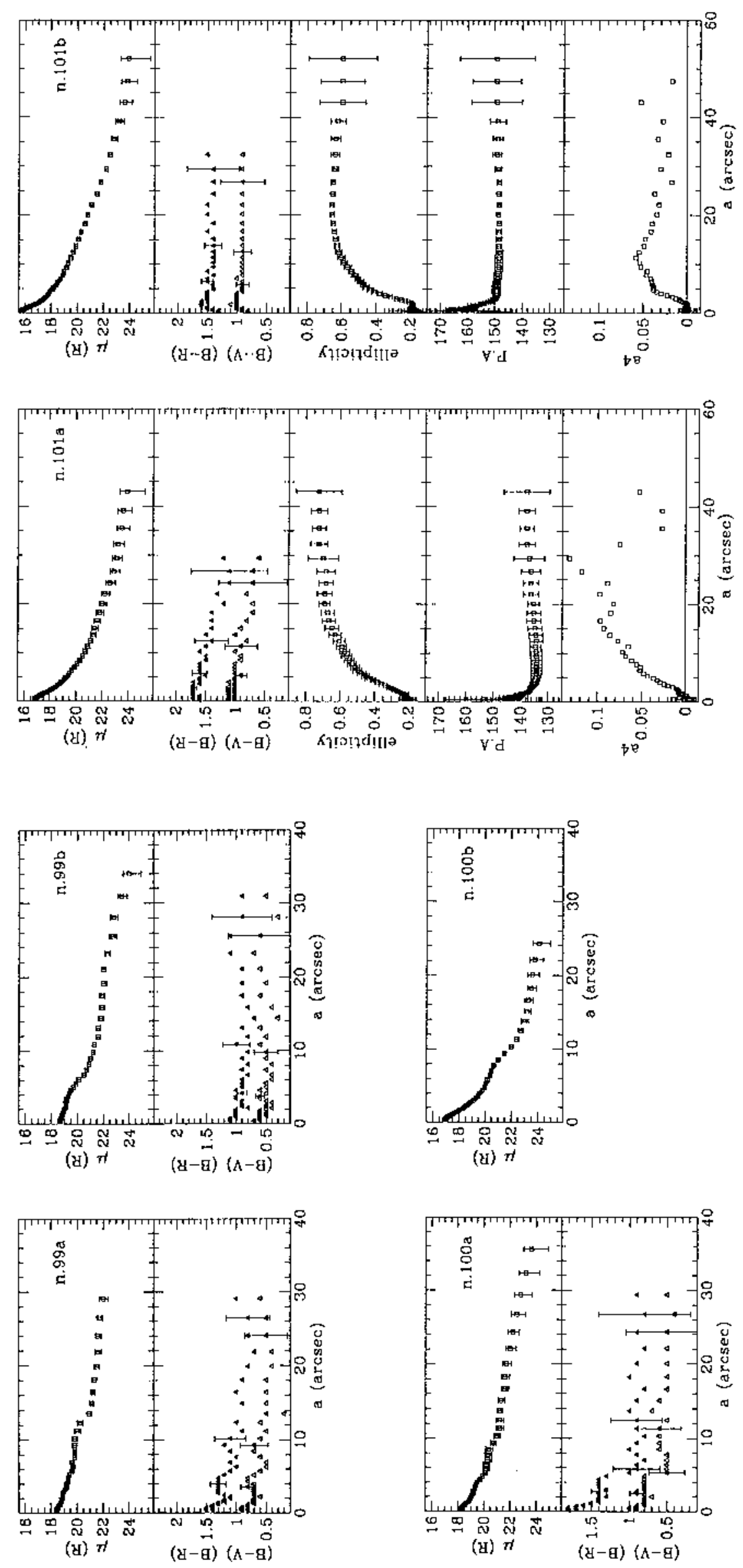

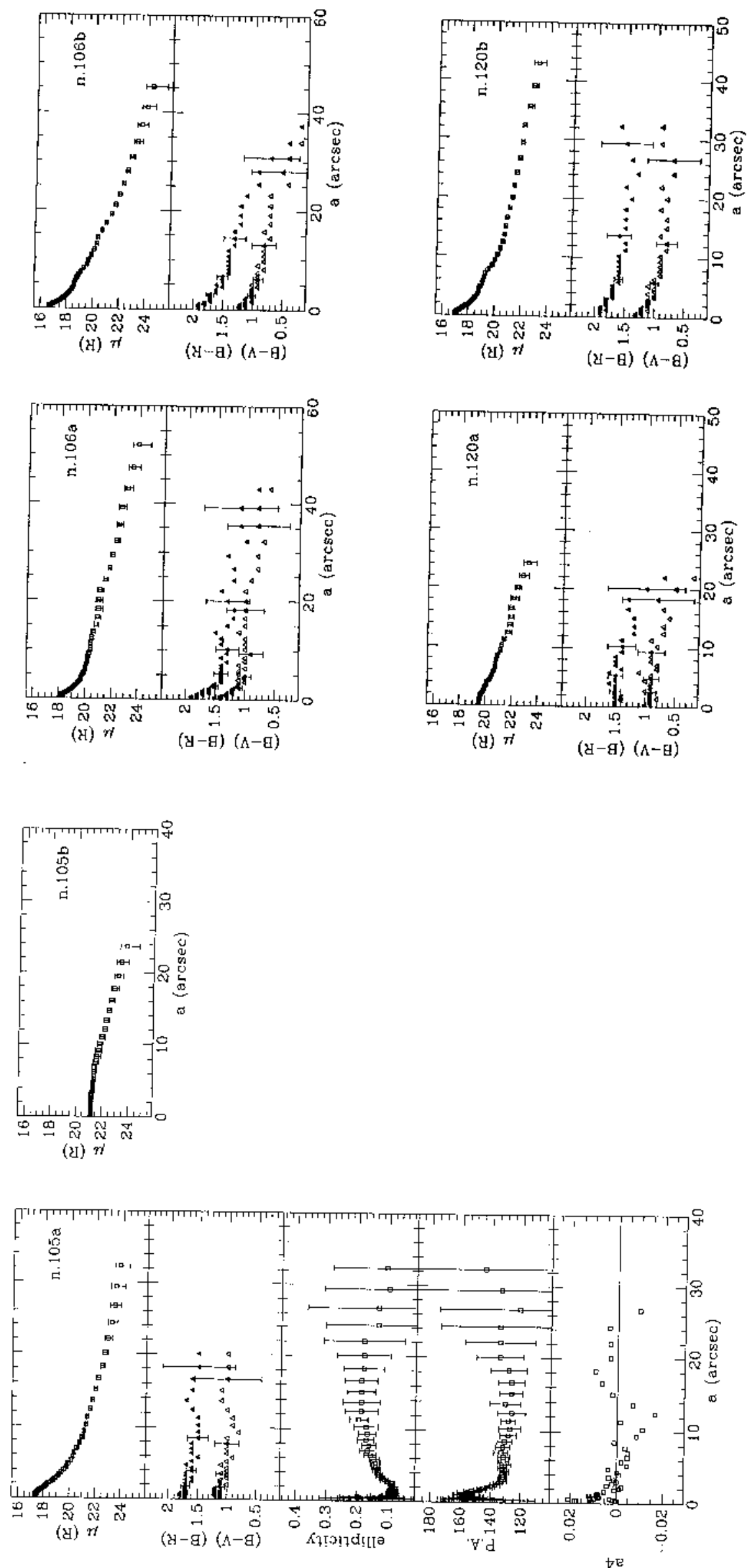

Fig. 3. continued. (To be seen in landscape) 

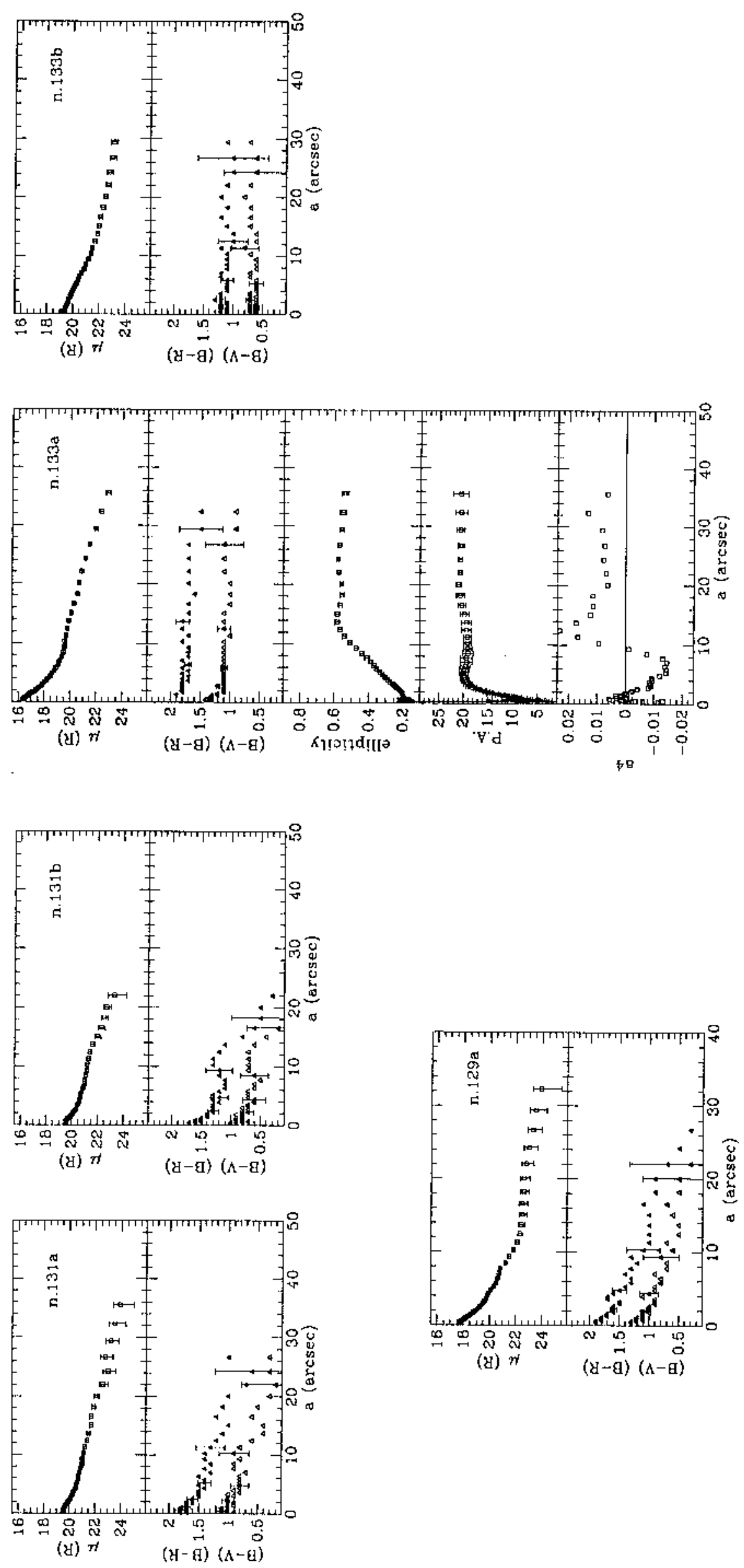

Fig. 3. continued. (To be seen in landscape) 

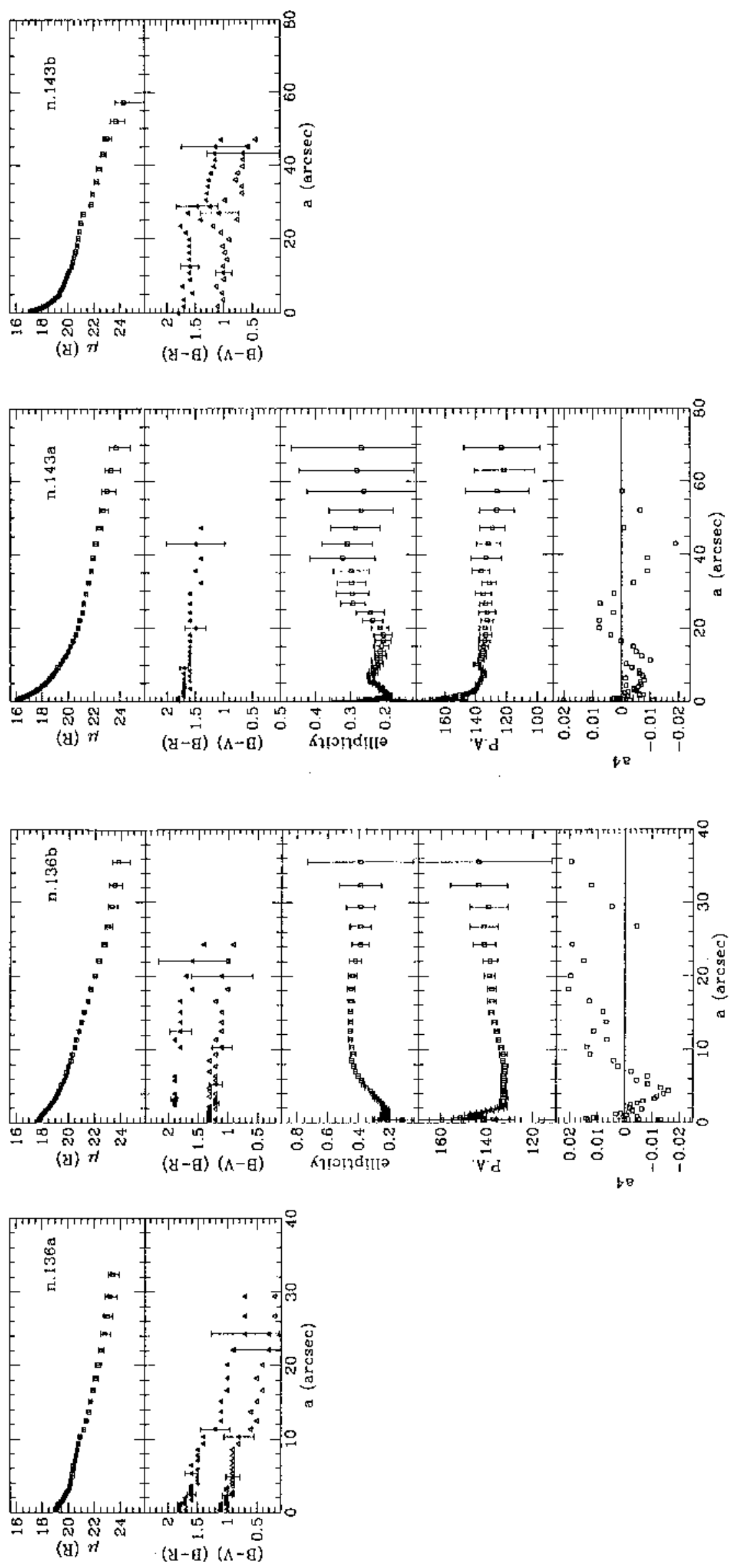

Fig. 3. continued. (To be seen in landscape) 

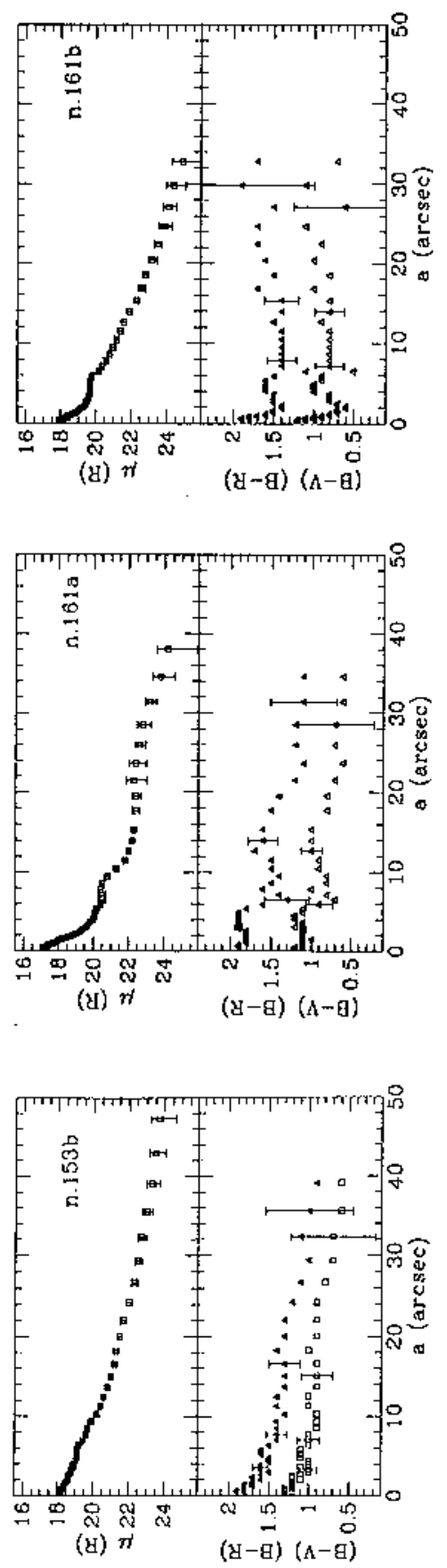
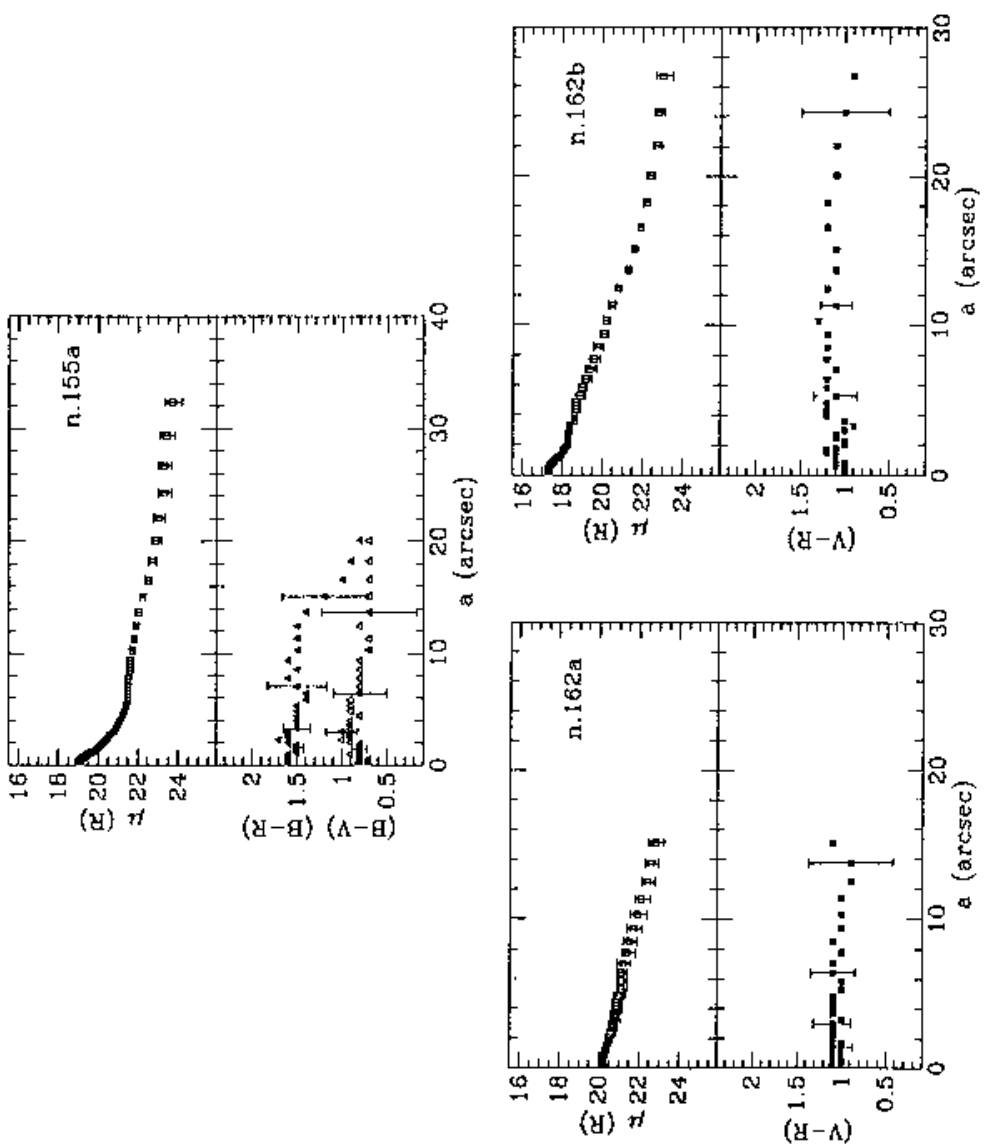

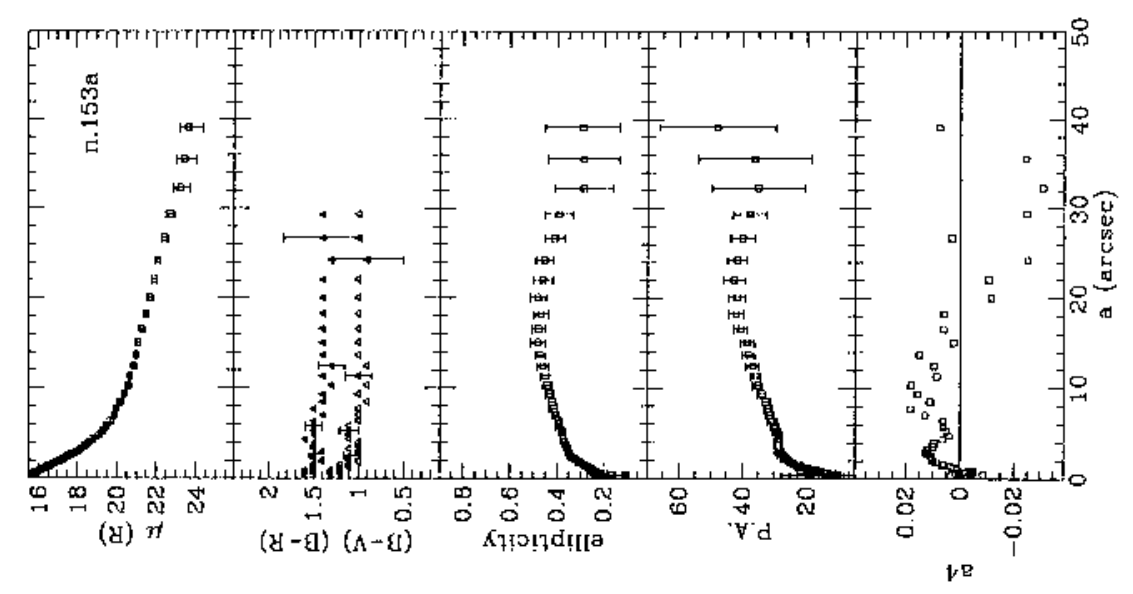



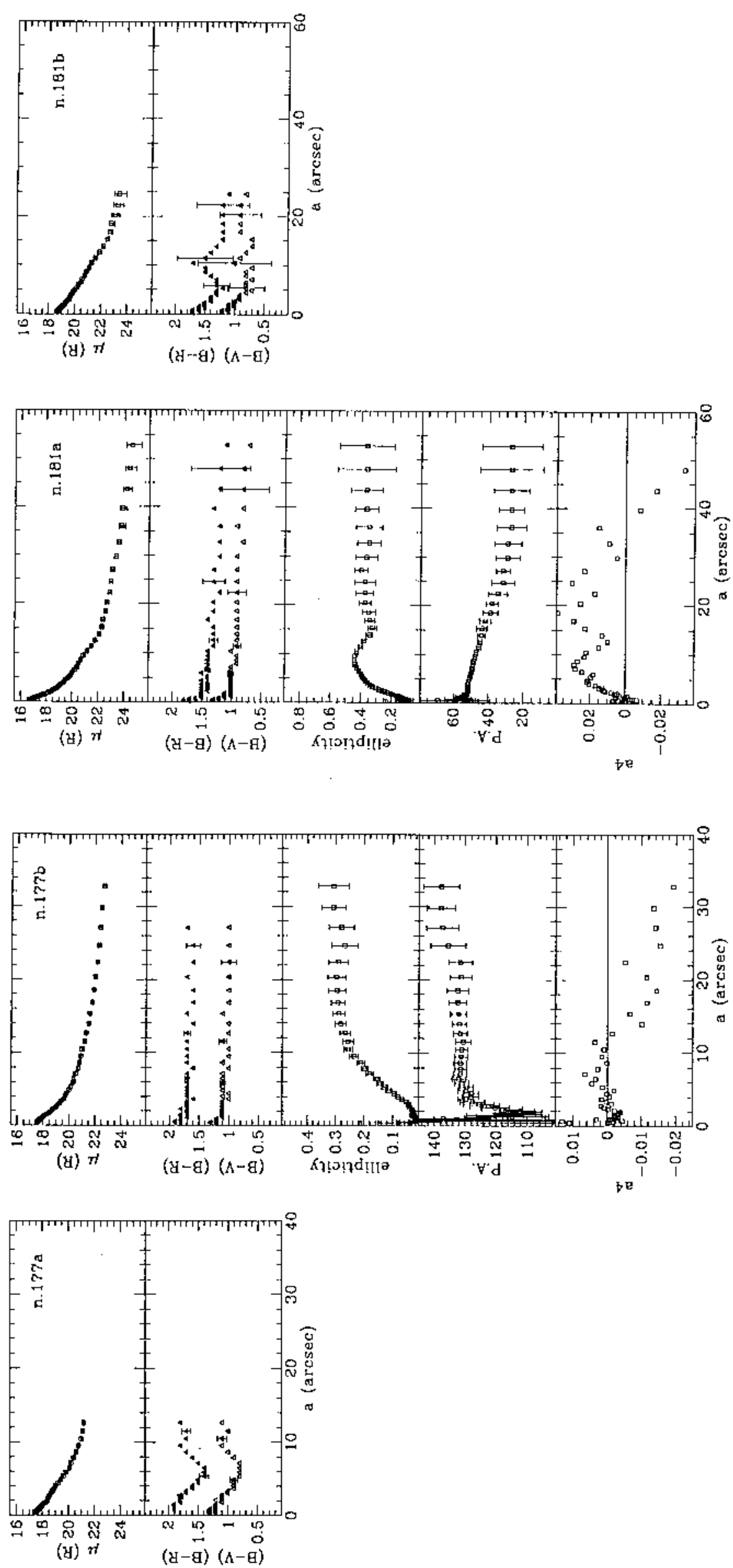

Fig. 3. continued. (To be seen in landscape) 

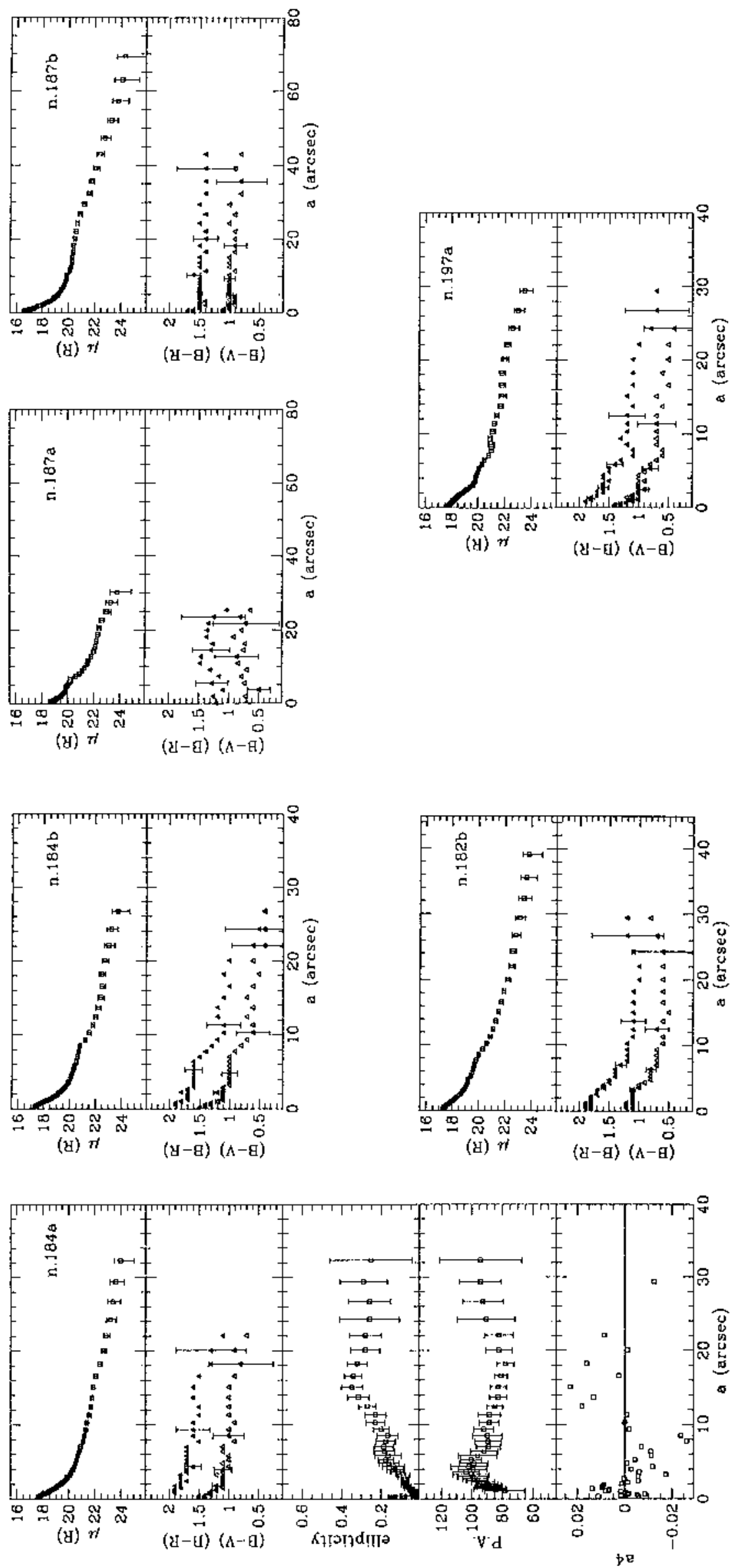

Fig. 3. continued. (To be seen in landscape) 

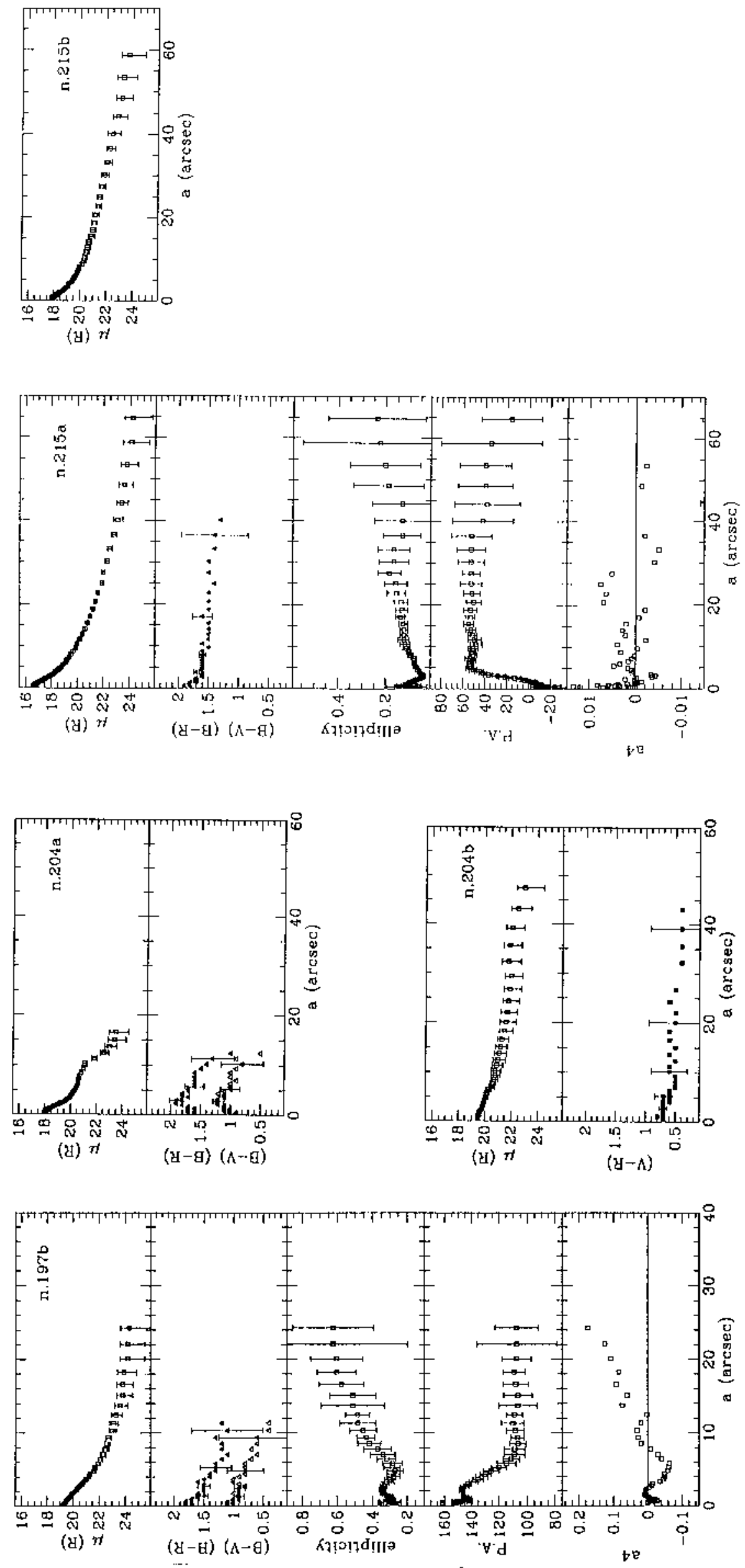

Fig. 3. continued. (To be seen in landscape) 

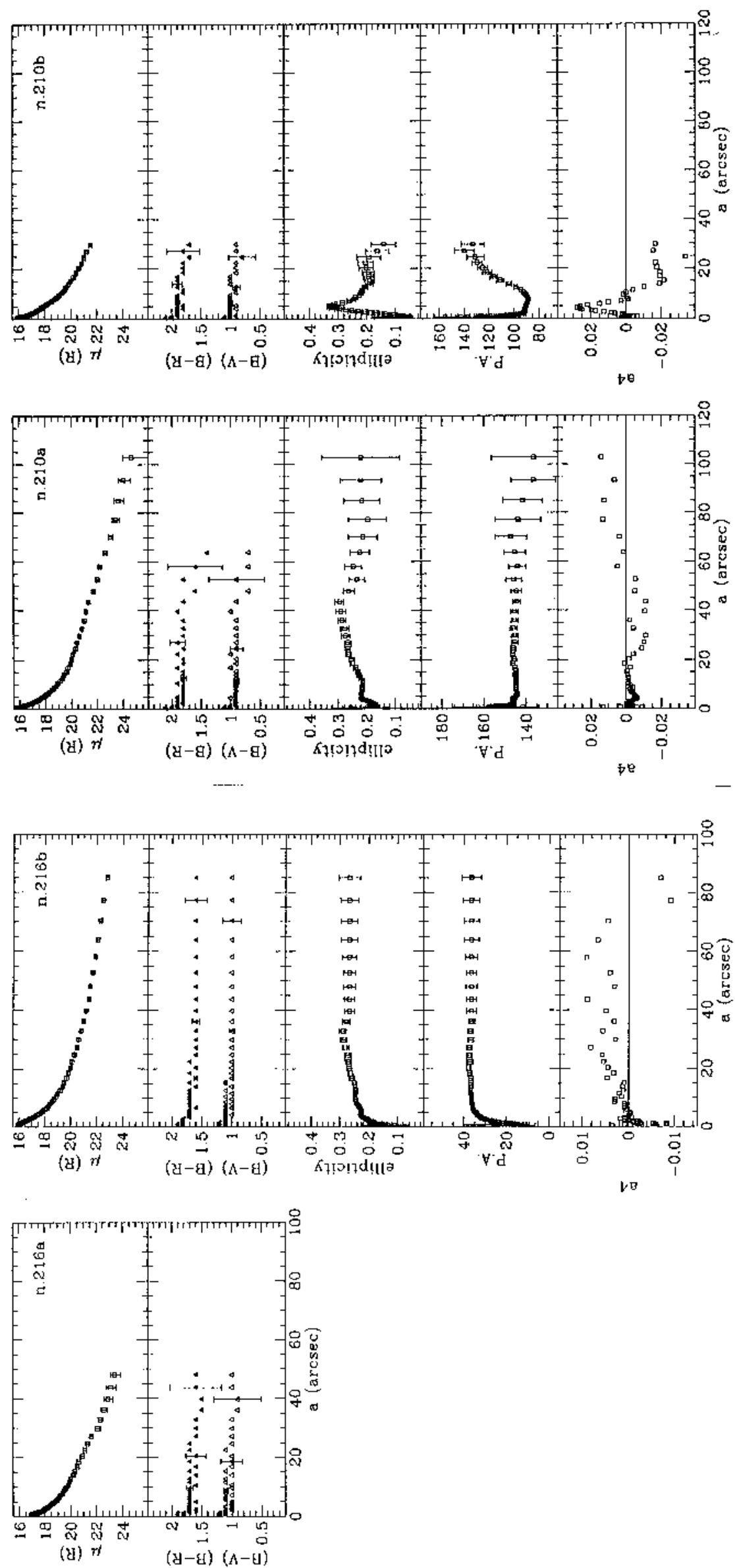

Fig. 3. continued. (To be seen in landscape) 

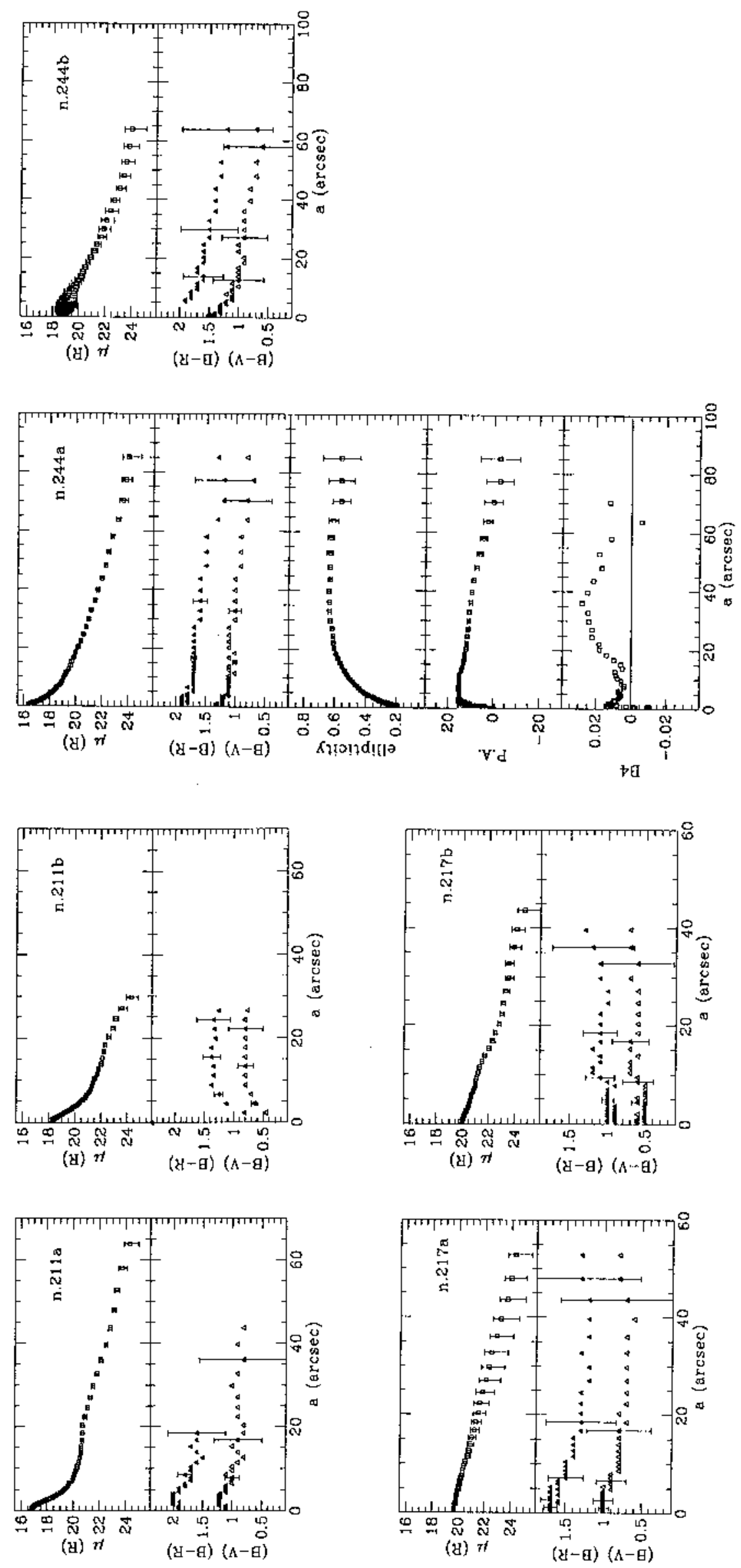

Fig. 3. continued. (To be seen in landscape) 

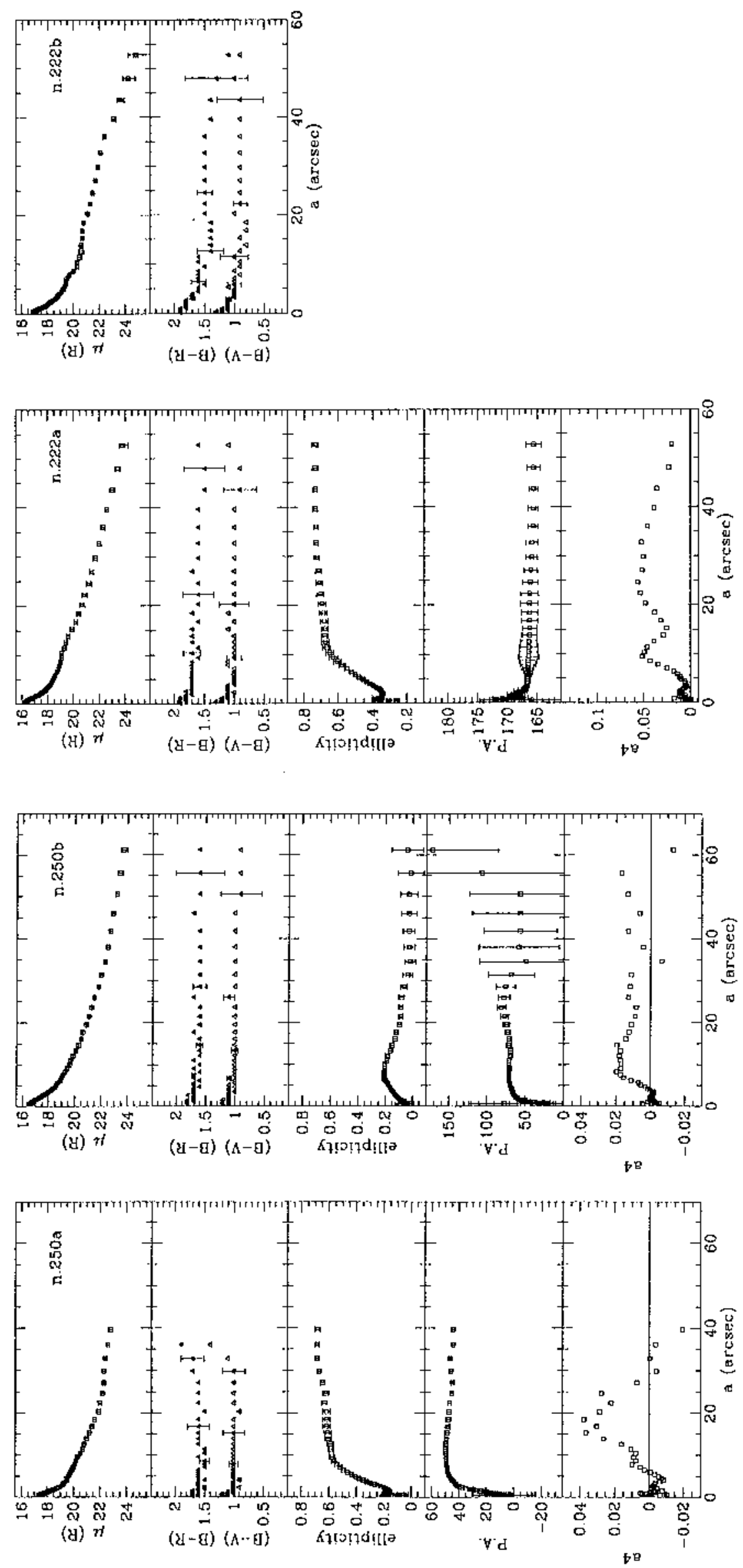

Fig. 3. continued. (To be seen in landscape) 

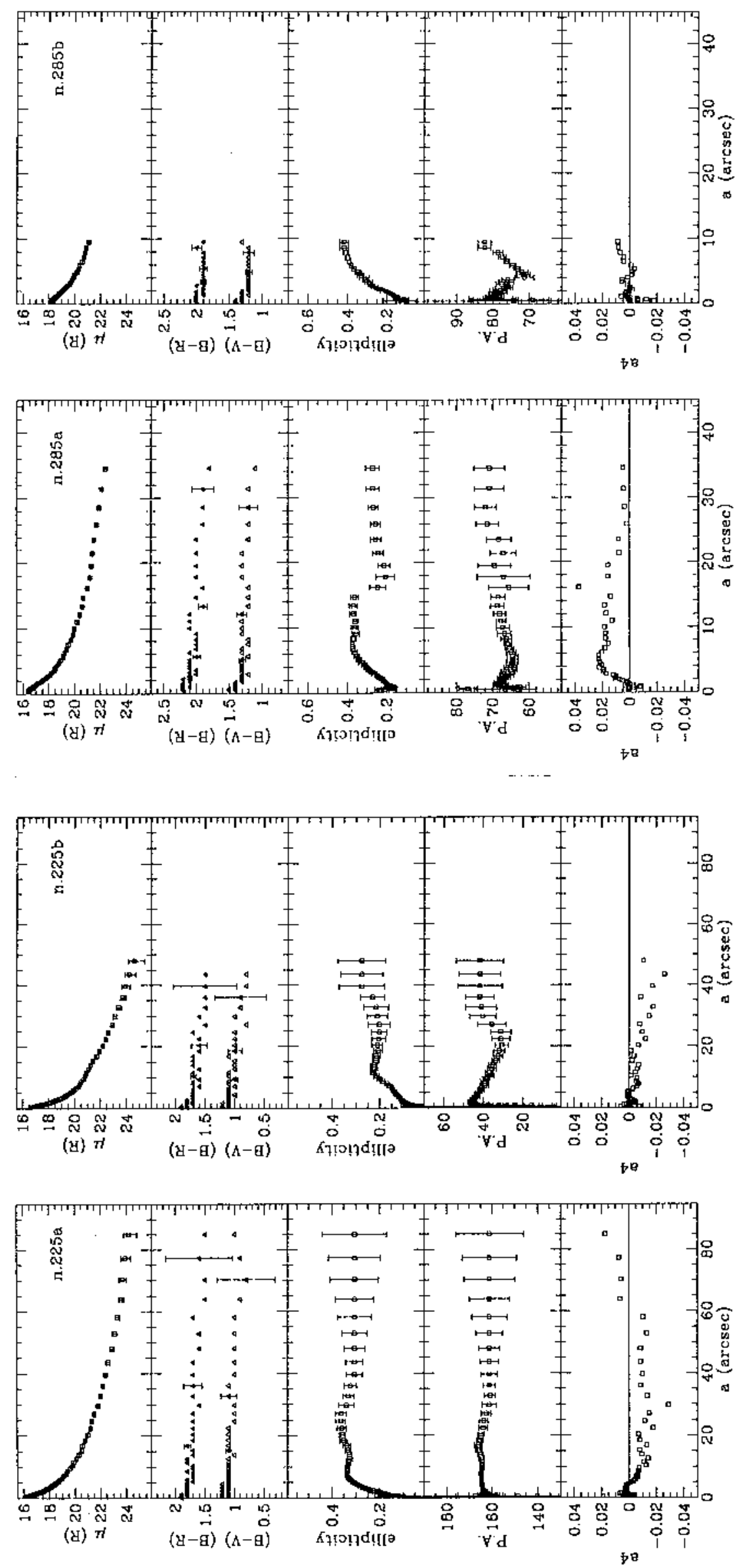

Fig. 3. continued. (To be seen in landscape) 

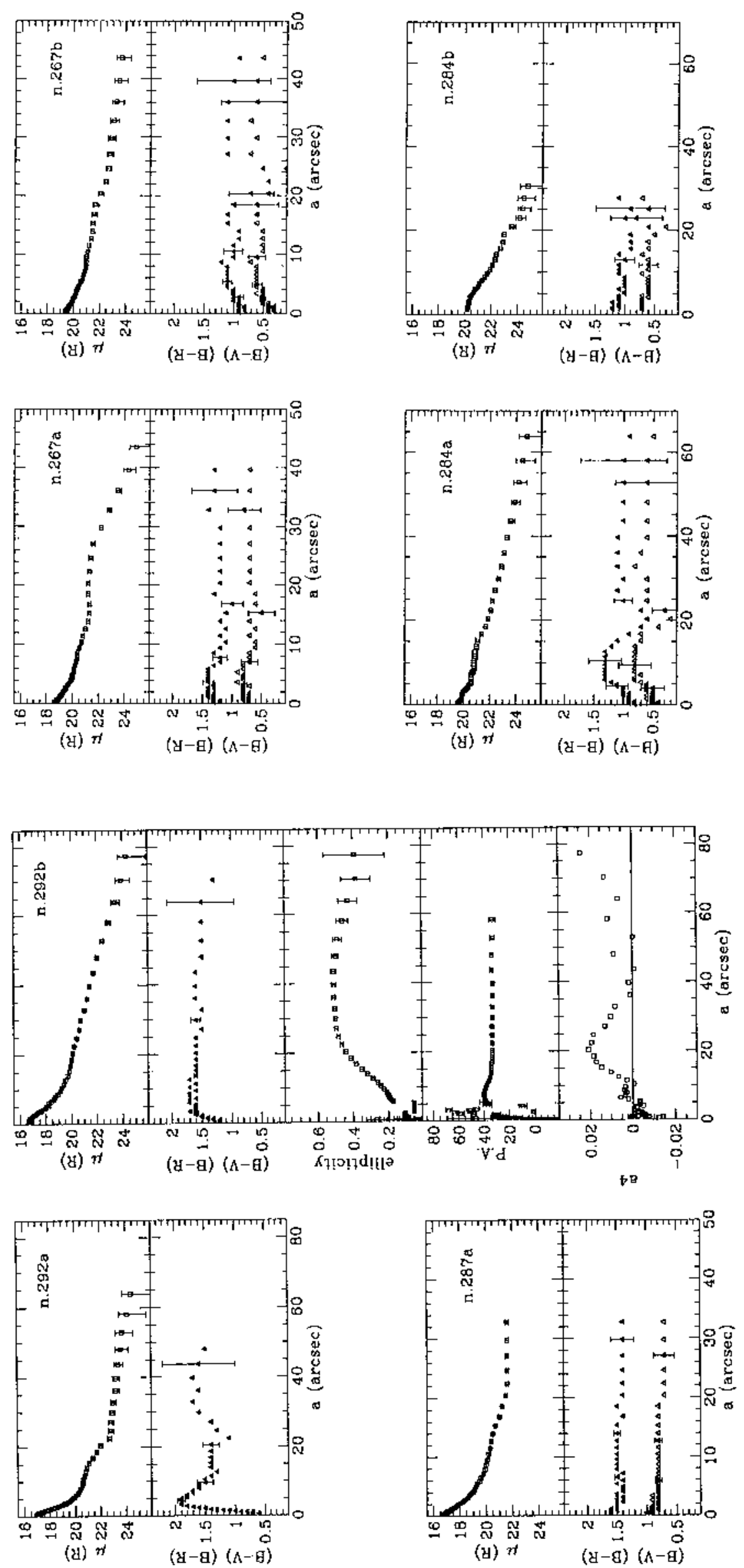

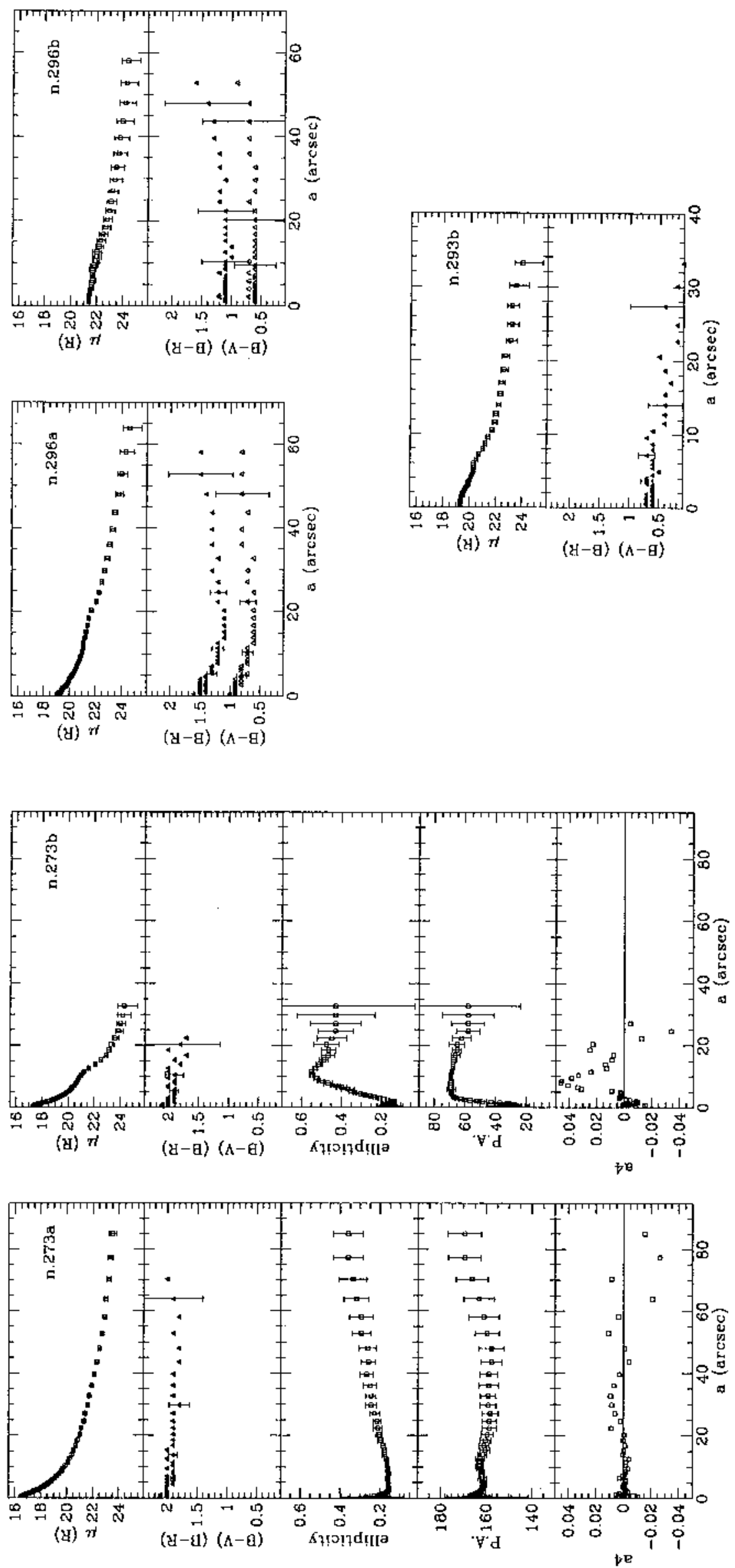

Fig. 3. continued. (To be seen in landscape) 

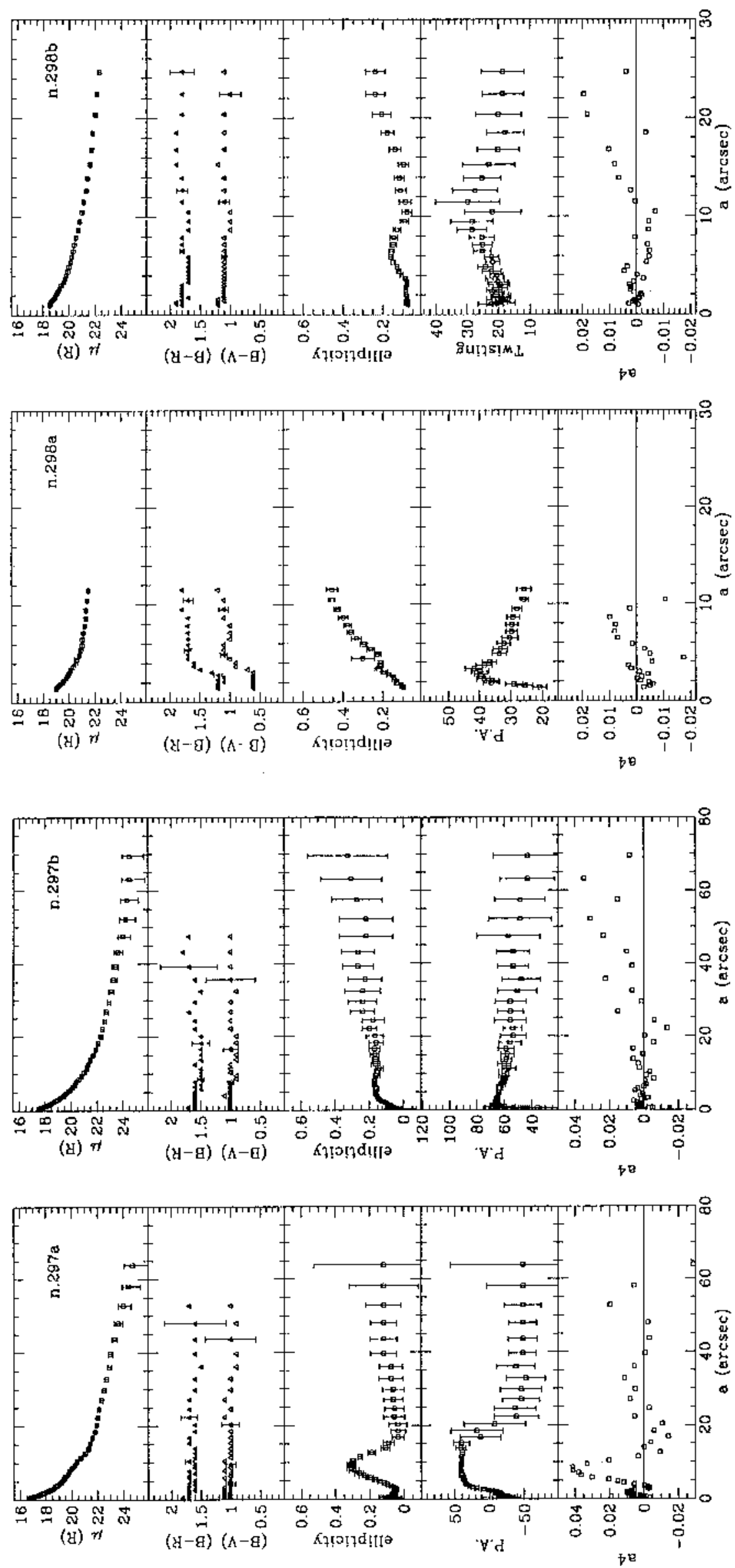

Fig. 3. continued. (To be seen in landscape) 

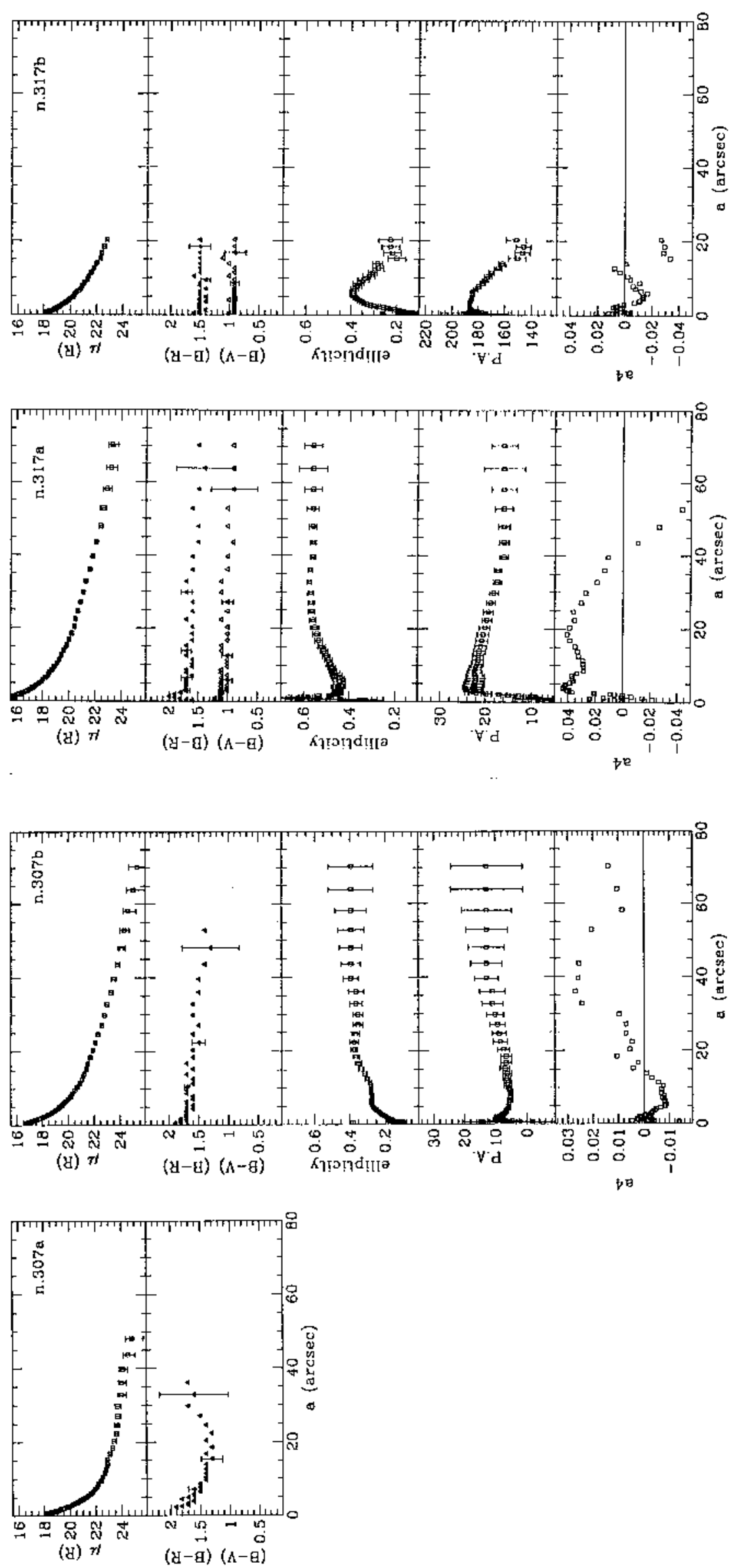

Fig. 3. continued. (To be seen in landscape) 

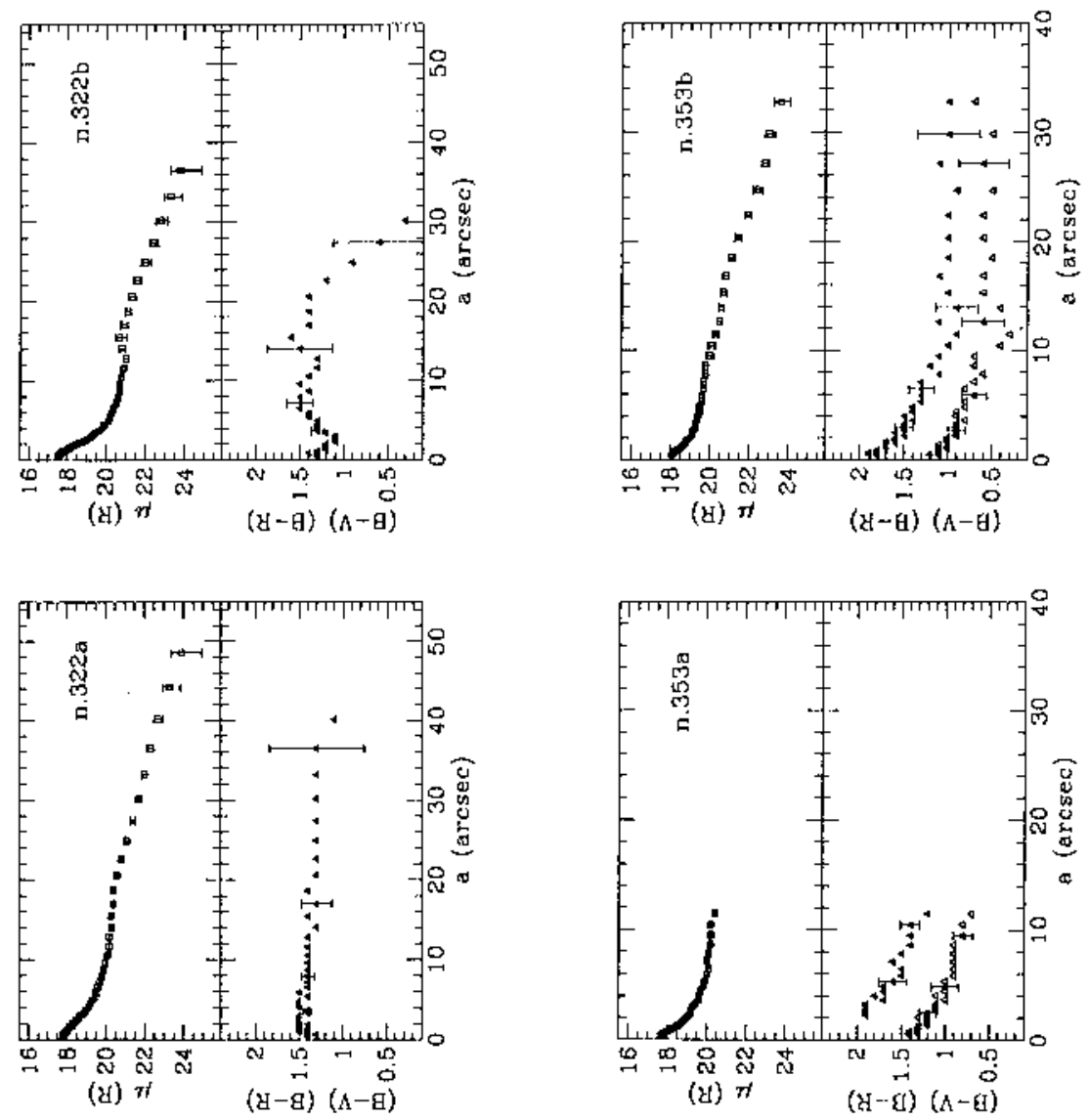

Fig. 3. continued. (To be seen in landscape) 

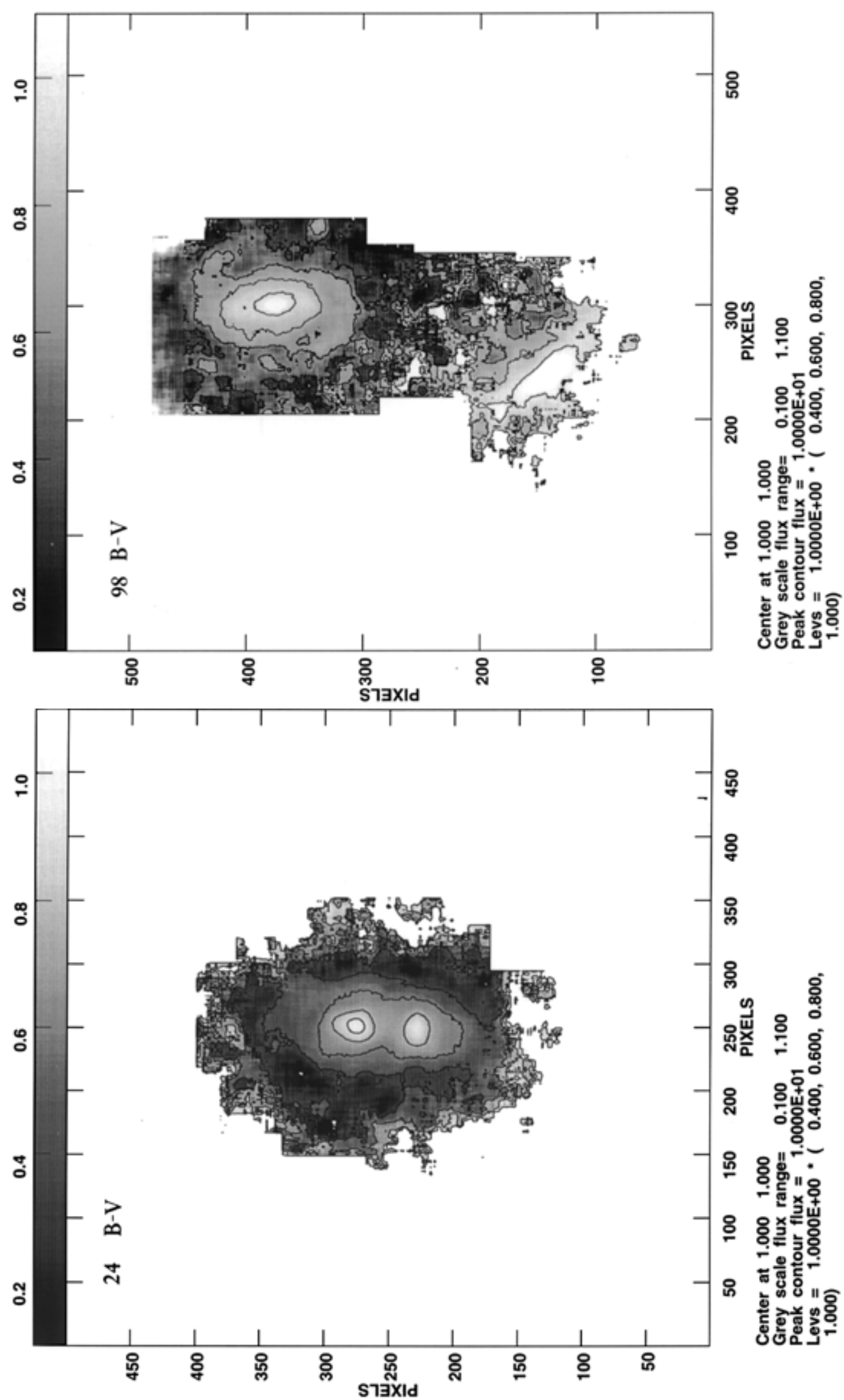

Fig. 4. $(B-V)$ or $(B-R)$ colour maps. The pixel size is $0.4 " / p x$. The grey scale an the correspondent colour value are given at the top of the figure. For $(B-V)$ the isophotal contours are: 0.4, 0.6, 0.8, 1.0; for $(B-R)$ are: 1.1, 1.3, 1.5, 1.7. (To be seen in landscape) 

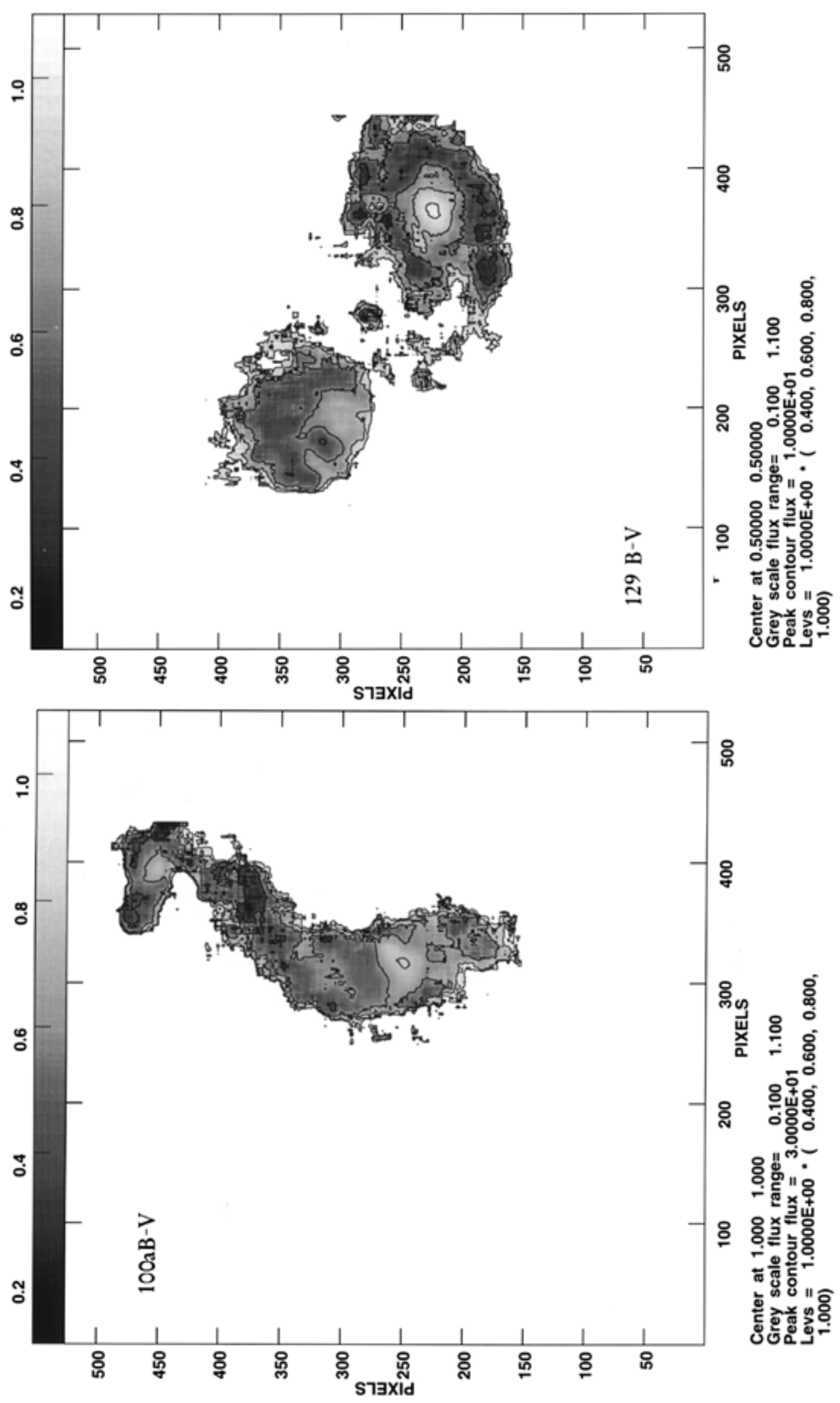

Fig. 4. continued. (To be seen in landscape) 

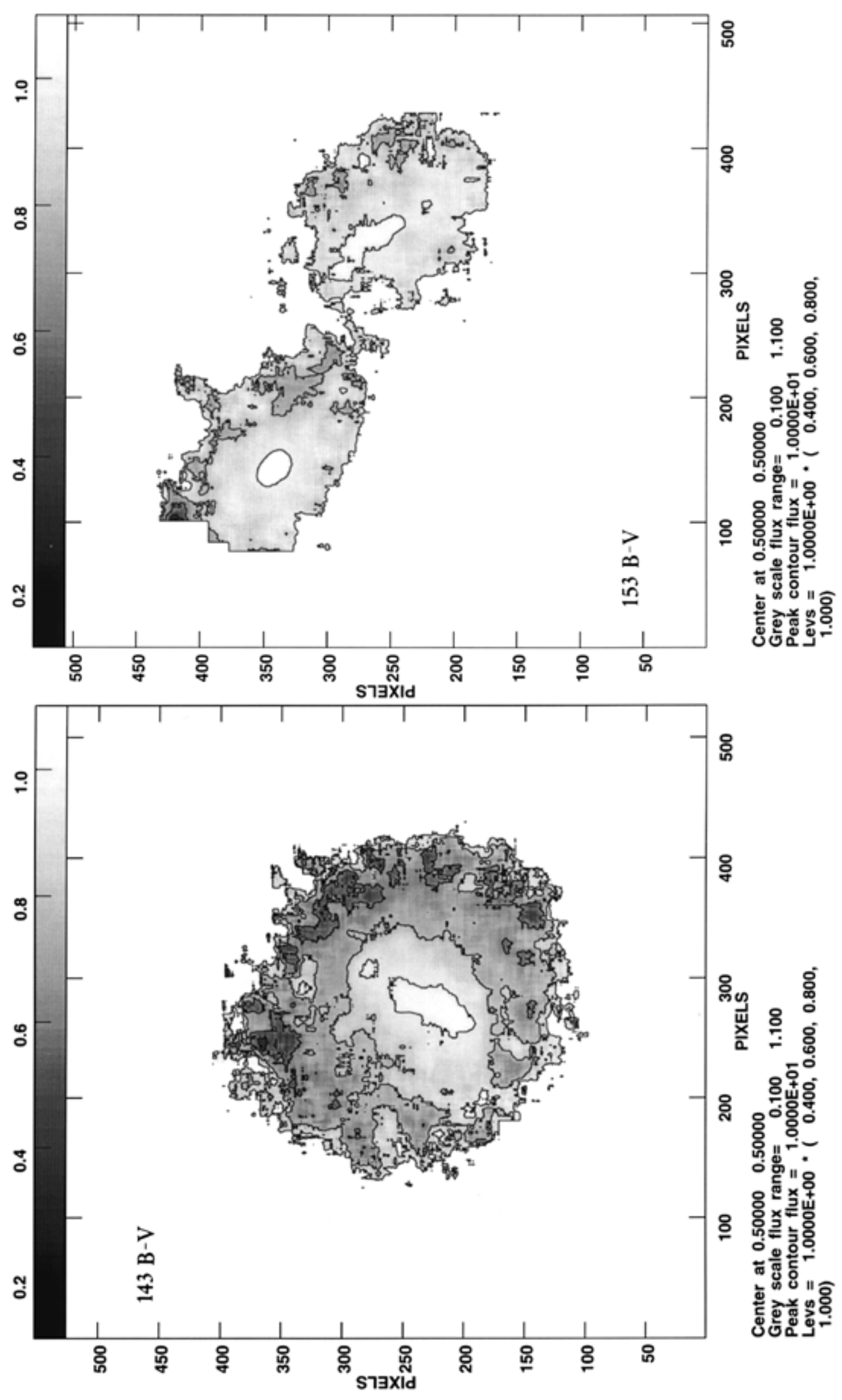

Fig. 4. continued. (To be seen in landscape) 

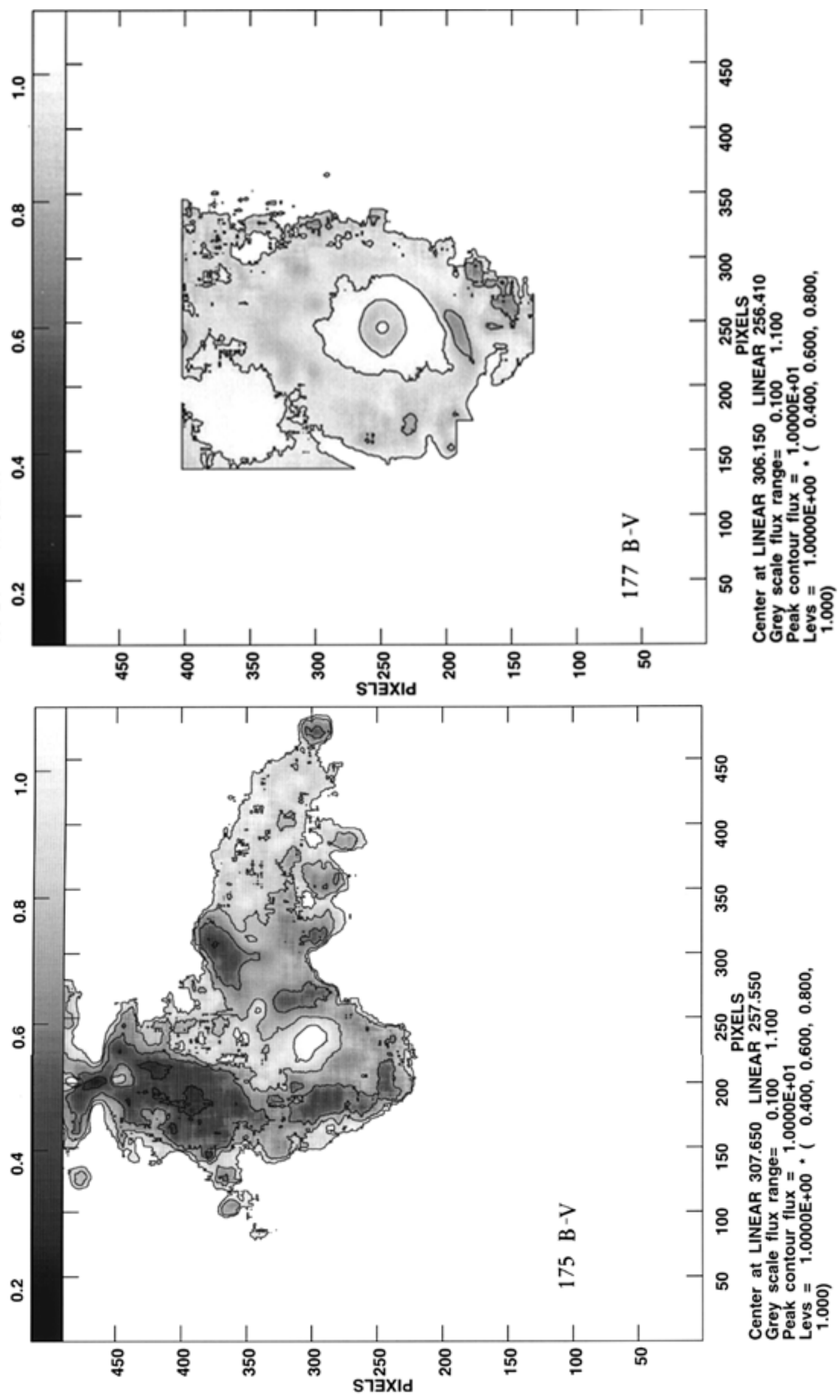

Fig. 4. continued. (To be seen in landscape) 

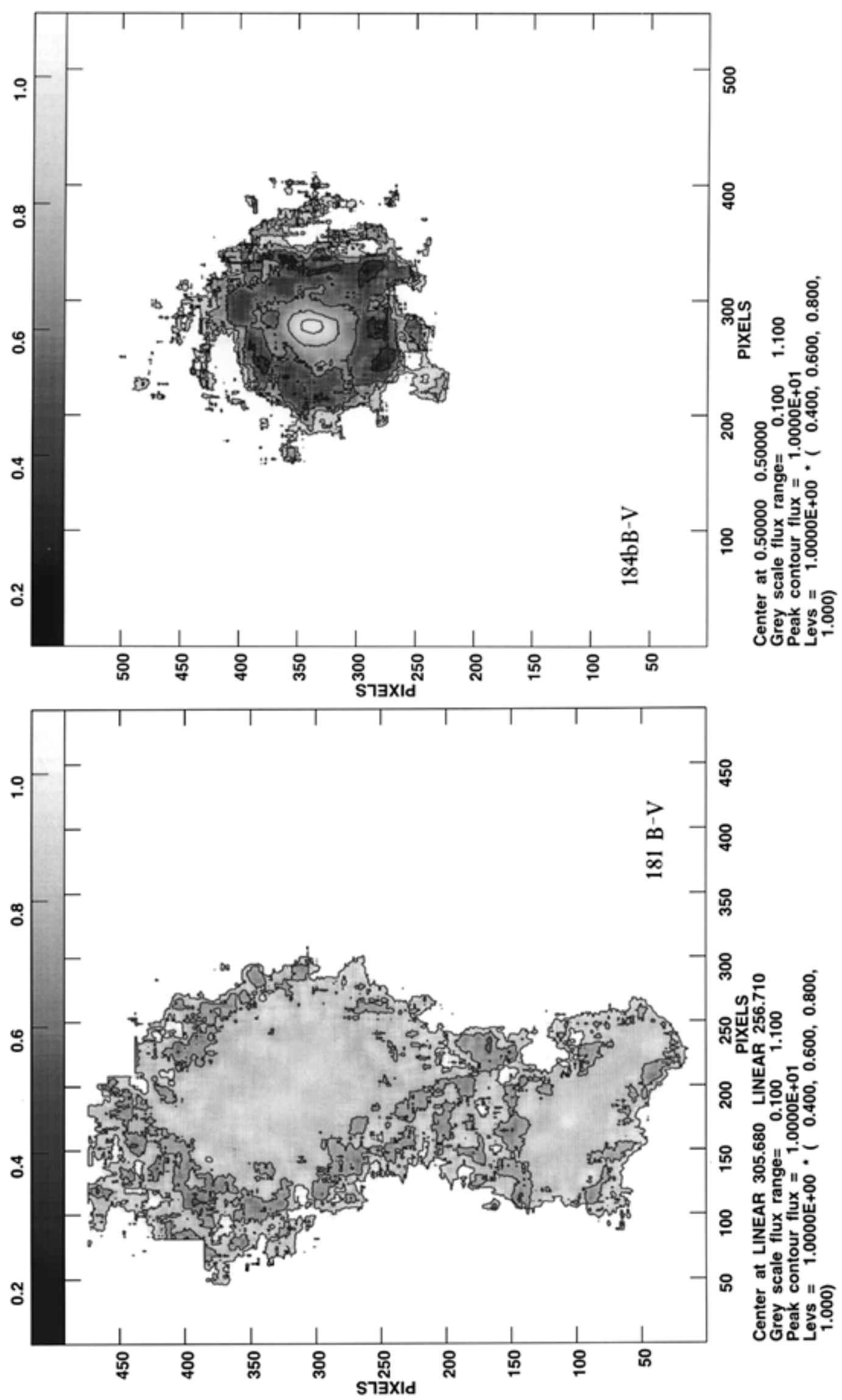

Fig. 4. continued. (To be seen in landscape) 

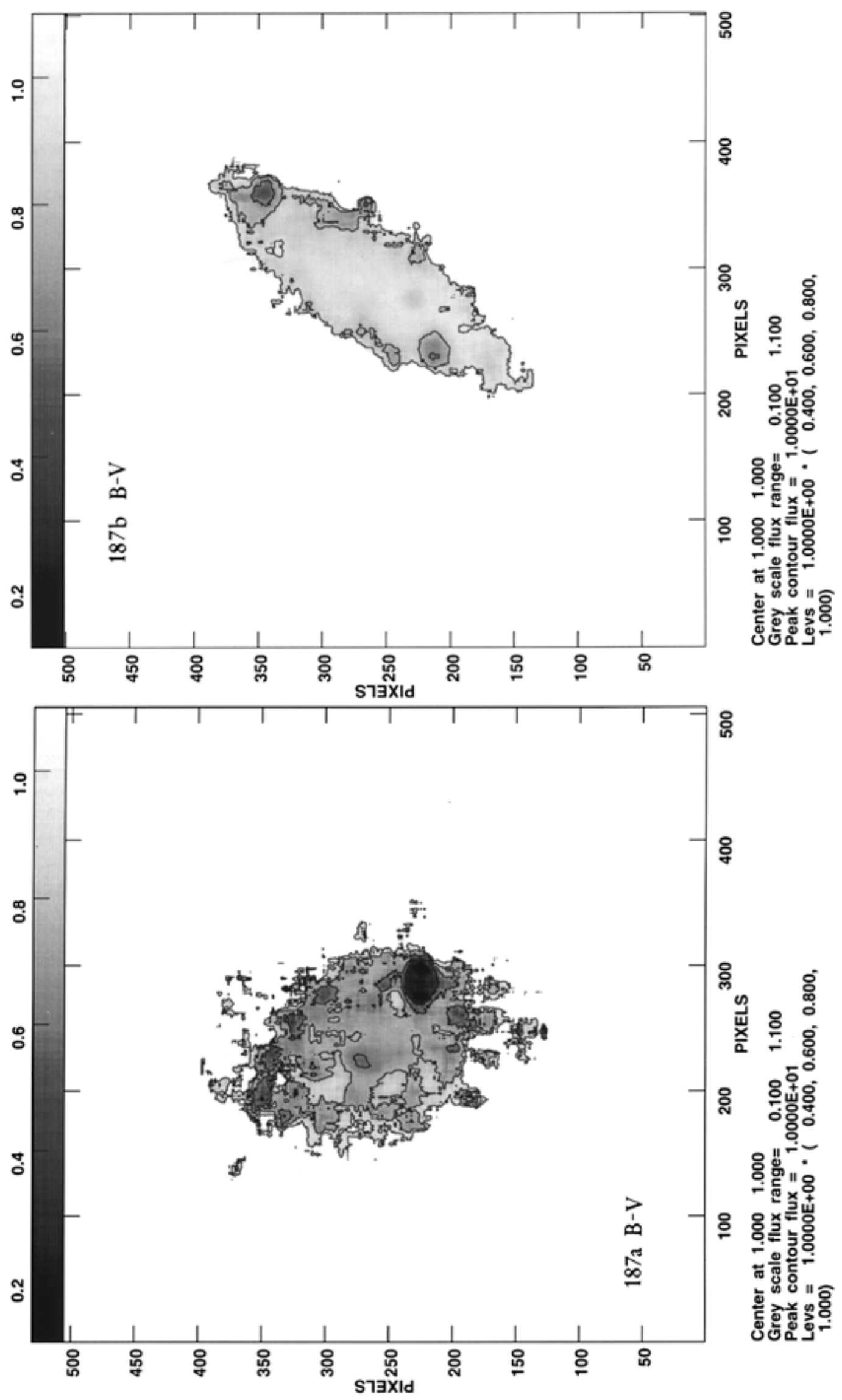

Fig. 4. continued. (To be seen in landscape) 

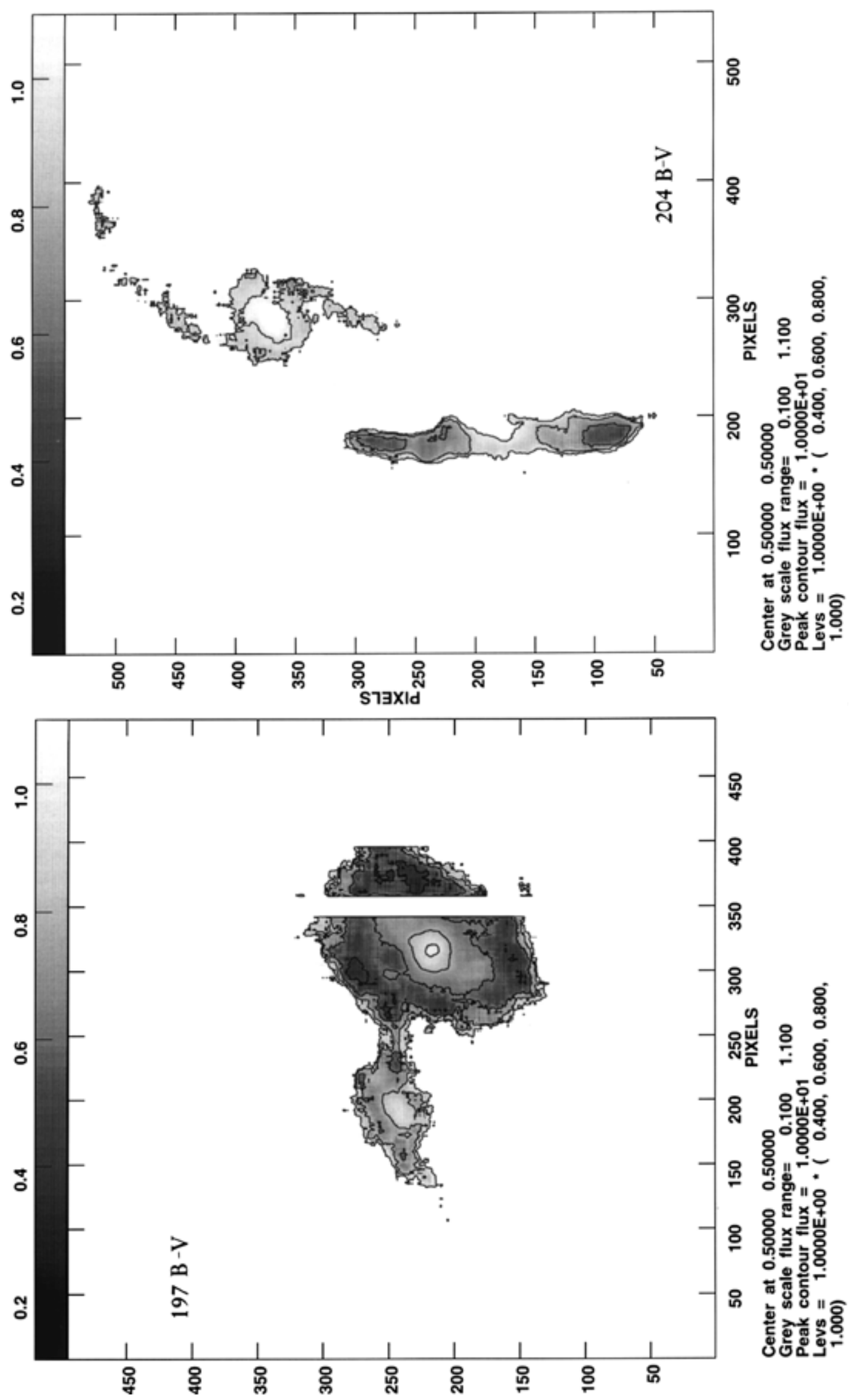

Fig. 4. continued. (To be seen in landscape) 

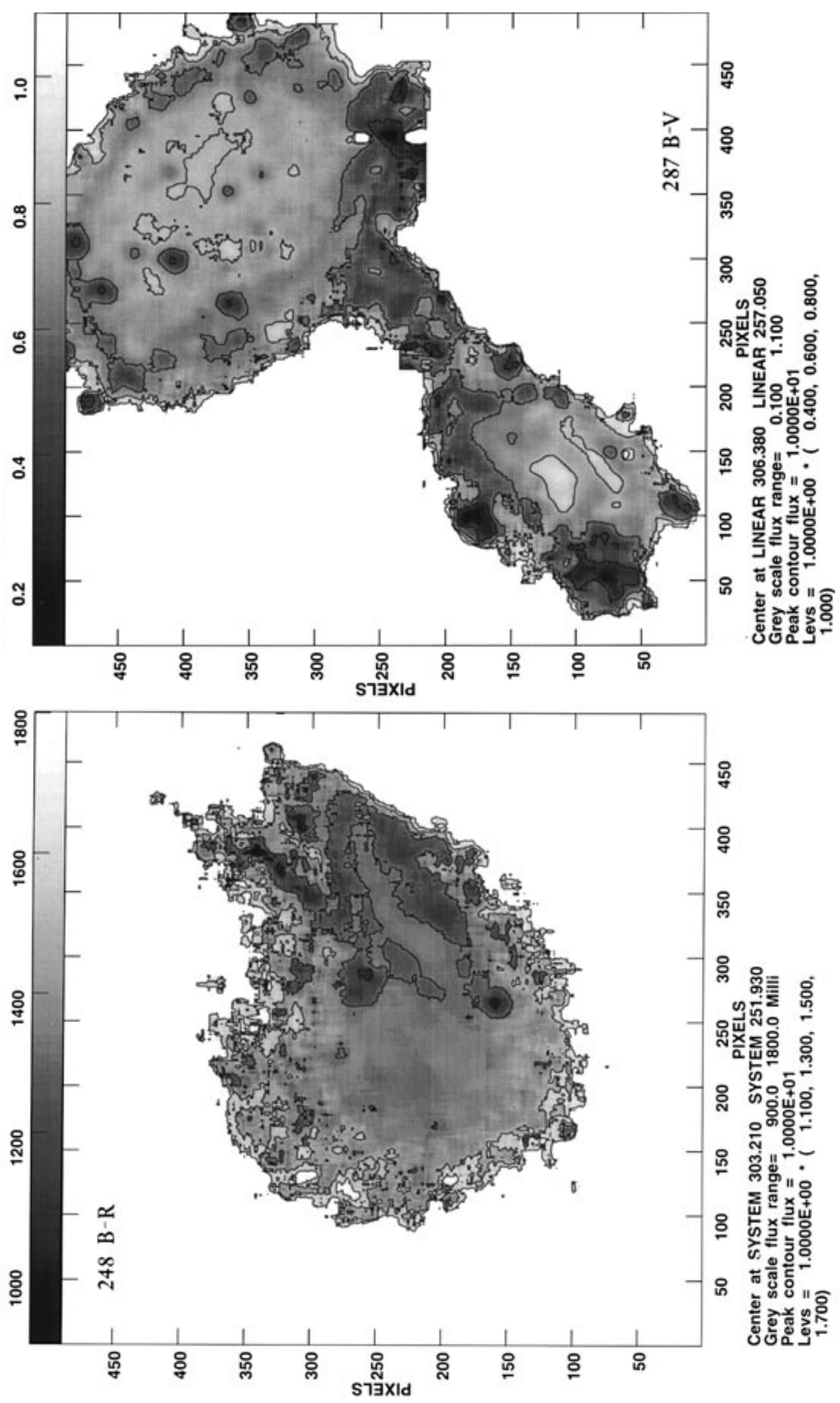

Fig. 4. continued. (To be seen in landscape) 

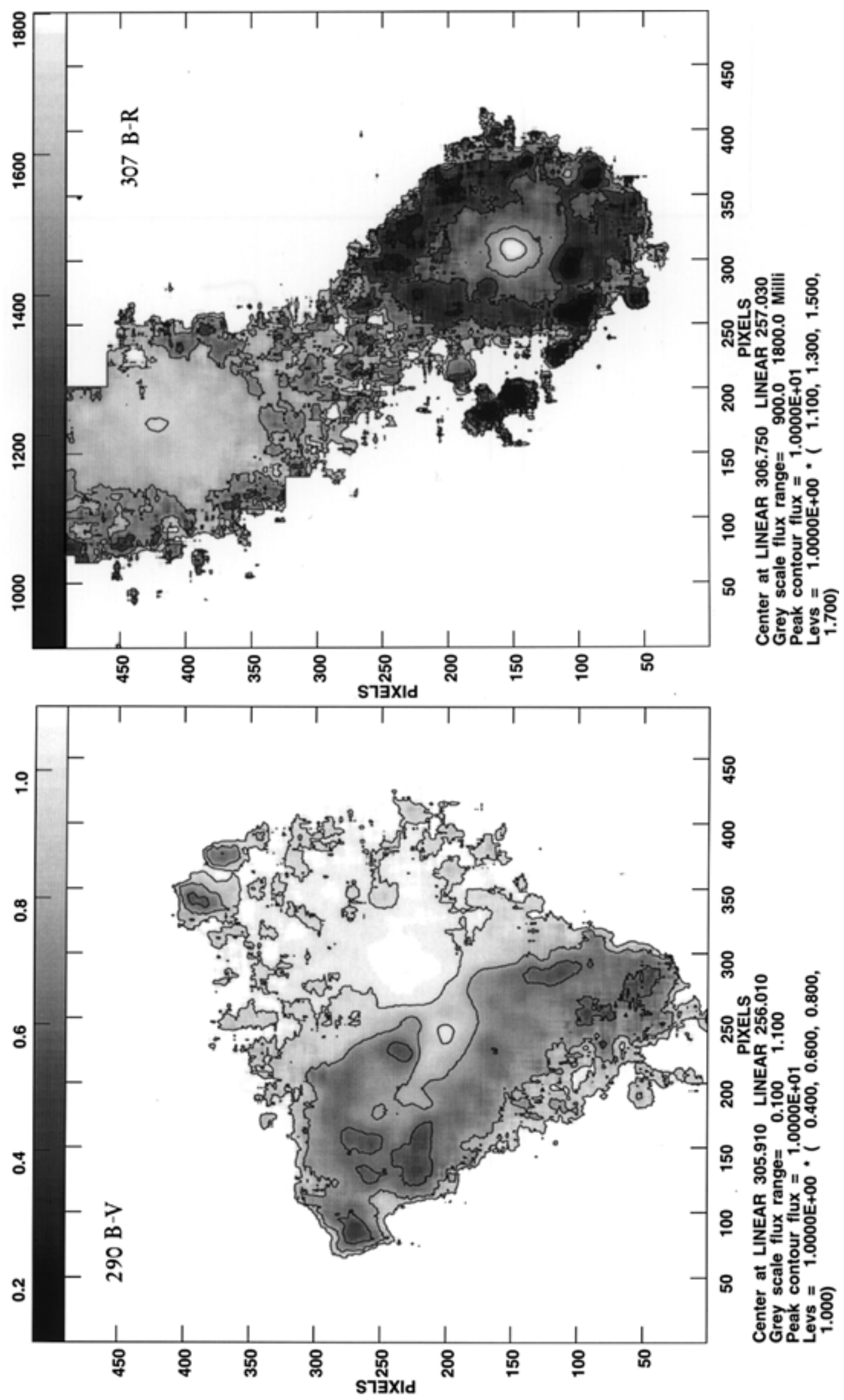

Fig. 4. continued. (To be seen in landscape) 


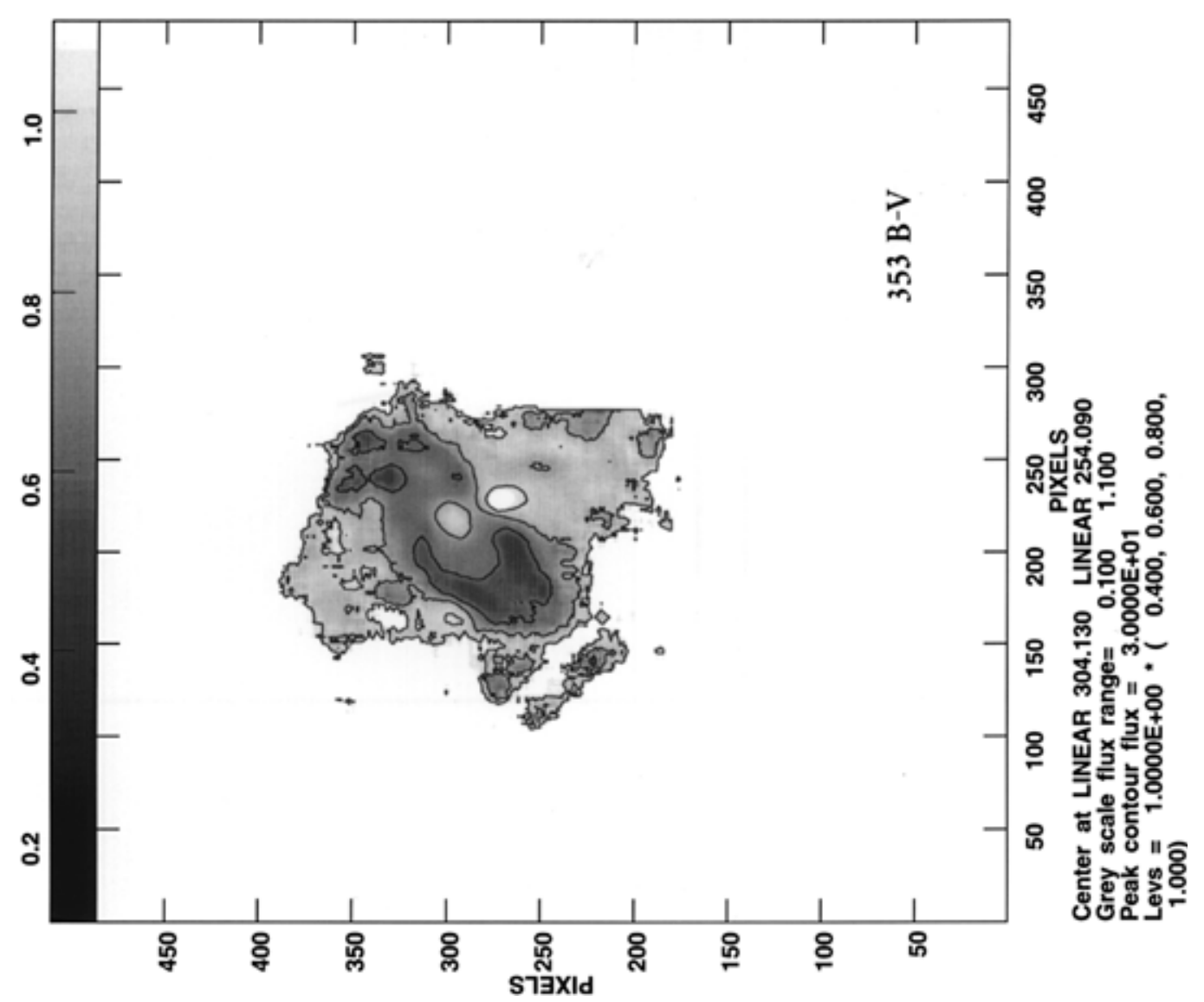

Fig. 4. continued. (To be seen in landscape) 